\title{
A geometric unification of dualities
}

\section{Citation}

Cachazo, F., B. Fiol, K. Intriligator, S. Katz, and C. Vafa. 2002. "A Geometric Unification of

Dualities." Nuclear Physics B 628 (1-2): 3-78. https://doi.org/10.1016/s0550-3213(02)00078-0.

\section{Permanent link}

http://nrs.harvard.edu/urn-3:HUL.InstRepos:41385085

\section{Terms of Use}

This article was downloaded from Harvard University's DASH repository, and is made available under the terms and conditions applicable to Other Posted Material, as set forth at http:// nrs.harvard.edu/urn-3:HUL.InstRepos:dash.current.terms-of-use\#LAA

\section{Share Your Story}

The Harvard community has made this article openly available.

Please share how this access benefits you. Submit a story.

Accessibility 
HUTP-01/A045

WIS/19/01-SEPT-DPP

UCSD-PTH-01-16

ILL-(TH)-01-9

hep-th/0110028

\title{
A Geometric Unification of Dualities
}

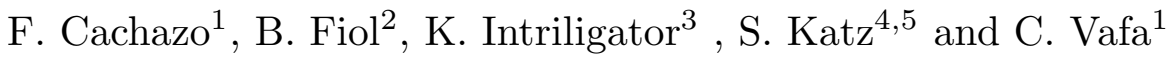 \\ 1 Jefferson Physical Laboratory, Harvard University, Cambridge, MA 02138, USA \\ ${ }^{2}$ Department of Particle Physics, The Weizmann Institute of Science, Rehovot, 76100, Israel \\ ${ }^{3}$ UCSD Physics Department, 9500 Gilman Drive, La Jolla, CA 92093 \\ ${ }^{4}$ Departments of Mathematics and Physics \\ University of Illinois at Urbana-Champaign, Urbana, IL 61801 USA \\ ${ }^{5}$ Department of Mathematics, Oklahoma State University, Stillwater, OK 74078 USA
}

\begin{abstract}
We study the dynamics of a large class of $\mathcal{N}=1$ quiver theories, geometrically realized by type IIB D-brane probes wrapping cycles of local Calabi-Yau threefolds. These include $\mathcal{N}=2$ (affine) A-D-E quiver theories deformed by superpotential terms, as well as chiral $\mathcal{N}=1$ quiver theories obtained in the presence of vanishing 4-cycles inside a Calabi-Yau. We consider the various possible geometric transitions of the 3-fold and show that they correspond to Seiberg-like dualities (represented by Weyl reflections in the A-D-E case or 'mutations' of bundles in the case of vanishing 4-cycles) or large $N$ dualities involving gaugino condensates (generalized conifold transitions). Also duality cascades are naturally realized in these classes of theories, and are related to the affine Weyl group symmetry in the A-D-E case.
\end{abstract}

October 2001 


\section{Introduction}

A deeper understanding of string theory on background geometries with some vanishing cycles has played a key role in various aspects of string dualities. An early example of this was in the context of the physical interpretation of the conifold [1] singularity and its possible transitions [2]. Geometric transitions have also played an important role in deriving field theoretic dualities from string theory. In particular by considering spacetime filling D-branes wrapped around cycles of Calabi-Yau 3-folds, Seiberg's duality was derived in this way in the context of type IIB [3] and type IIA [W] string theories.

Geometric transitions have also played a key role in large $N$ dualities. The AdS/CFT correspondence [5] can be viewed as an example of such a transition [6], where before the transition (small 't Hooft parameter) there are D-branes wrapped around cycles, and after the geometric transition (large 't Hooft parameter) these cycles which supported the D-branes have disappeared, and have been replaced with flux through a dual cycle. The large $N$ duality of Chern-Simons with topological strings [6] is an example of this kind. The geometric transition duality was embedded in type IIA superstring [7], with D6 branes wrapping an $S^{3}$ on one side of the transition and fluxes through a dual $S^{2}$ on the other side; this leads to a large $N$ duality for $\mathcal{N}=1$ Yang-Mills theory in 4 dimensions. This duality was lifted up to M-theory [8,9] where it was interpreted as a purely geometric transition. Since, as argued in [9] and further elaborated in [10,11] the transition in quantum geometry is smooth in the M-theory lift, this leads to a derivation of the geometric transition duality.

The type IIB mirror of these large $N$ dualities has also been studied [12,13] (see also [14]). One aim of this paper is to generalize these constructions and show that the Seiberglike dualities and large $N$ dualities/gaugino condensation can be viewed in a unified way as geometric transitions in the same setup. We consider a wide variety of $4 \mathrm{~d}, \mathcal{N}=1$ supersymmetric gauge theories, which can be constructed via branes which partially wrap cycles of a (non-compact) Calabi-Yau 3-fold $X$. In type IIB, one can consider general combinations of $D 3, D 5, D 7$ branes, wrapped over various cycles and filling the 4 dimensional spacetime. This generically leads to a theory with gauge group $\prod_{i} U\left(N_{i}\right)$ with some matter in the bifundamental representations, and some superpotential terms (depending on the complex structure of $X$ ). Changing the Kahler parameters of the underlying CY 3 -fold translates to changing the coupling constants of the gauge theory (and sometimes also to FI terms).

We find, as in [3, that changing the Kahler parameters of $X$ (or, in the type IIA mirror with wrapped D6 branes, changing the complex parameters) changes the description 
of the gauge theory. As we pass through transitions in the 3-fold geometry, corresponding to blowing up different Kahler classes, we find dual gauge theory descriptions of the same underlying theory. These are transitions where some 2- or 4-cycles shrink and other 2- or 4-cycles grow. On the other hand, for a class of these theories which eventually confine, with gaugino condensates, we find transitions of the type where the 2-cycle or 4-cycle has shrunk, disappeared, and instead a number of finite size $S^{3}$ 's have emerged, with fluxes through them. The description in terms of the blown up $S^{3}$ 's is better at large $N$ (in the IR), where the size of the $S^{3}$ s, which corresponds to the gaugino condensation, is large.

By using the holographic picture, and following the geometric transitions, we can smoothly follow the field theory dualities and dynamics along the renormalization group flow. In the UV, which corresponds to far distance to the geometry, we have a description which is best given in terms of finite size 2-cycles and 4-cycles. This is the weak coupling limit. The renormalization group flow to the IR corresponds in the geometry to going towards the tip of the cone (or more precisely towards the "tips" of the cone). In doing so, the description changes: some 2-cycles or 4-cycles shrink, and others emerge, corresponding to Seiberg-like dualities in the field theory. Eventually the gauge theory flows to e.g. a RG fixed point, a free-magnetic phase, or confinement with gaugino condensation. This is seen by following the geometry towards the tips of the cone. E.g. deep in the IR, or in the very large $N$ limit, the description might be best in terms of the blown up $S^{3}$ 's; this is where the gauge theory confines and gaugino condensation has taken place.

In this way we have a unified geometric picture, where both kinds of dualities can be seen in the same RG trajectory, depending on where in the geometry we are. This unification sharpens the picture of Seiberg duality given in [3, 4] (a similar comment applies to the brane construction of [15]): Rather than just seeing that two gauge theories are connected by changing the moduli of the theory, which by itself is not a complete derivation of duality 1 ) we can use the geometry to follow the RG trajectory, and see which description is best, at which scale, as we flow to the IR.

We consider two classes of local 3-folds in type IIB. One type (i) involves certain Calabi-Yau threefolds which only has compact 2-cycles and no compact 4-cycles. The

1 For example, by similar changes of the moduli one can relate $\mathcal{N}=2 U\left(N_{c}\right)$, with $N_{f}$ flavors, to $\mathcal{N}=2 U\left(N_{f}-N_{c}\right)$ with $N_{f}$ flavors. But here this duality misses part of the story. The original $U\left(N_{c}\right)$ theory does indeed contain the free-magnetic $U\left(N_{f}-N_{c}\right)$ theory in its spectrum, but this description is only good on part of the Higgs branch, and it also must be augmented with an extra $U(1)^{2 N_{c}-N_{f}}$ where this Higgs branch part intersects the Coulomb branch [16]. 
other type (ii) involves Calabi-Yau's which have compact 2- and 4-cycles. For type (i) we consider $X$ to have the geometry of an A-D-E 2-fold geometry fibered over a plane, with some blown up 2-cycles $S_{i}^{2}$ 's in one to one correspondence with the simple roots of A-D-E. We can wrap D5 branes over these 2-cycles, which fill the directions transverse to $X$. In addition, one could also include $N_{0}$ additional D3 branes transverse to $X$. The 3 -folds $X$ which we consider can thus be labelled (up to deformations) as $X(k, G)$ with $G$ the A-D-E group and $k$ an integer which labels the data about how the holomorphic 2-cycles of the A-D-E are fibered over the plane [17].2 For $N_{0}=0$, the gauge groups obtained via wrapping various numbers $N_{i}$ D5 branes over the various $S_{i}^{2}$ of $X(k, G)$ are quiver gauge theories with gauge group $\prod_{i=1}^{r} U\left(N_{i}\right)$, with the quiver diagram the $G$ Dynkin diagram and $r=\operatorname{rank}(G)$, and the matter in hypermultiplets dictated by the links of the Dynkin diagram. The theory arises from the corresponding $\mathcal{N}=2$ quiver theory, broken to $\mathcal{N}=1$ by the additional superpotentials for the adjoint superfields $\phi_{i}$ in the $\mathcal{N}=2 U\left(N_{i}\right)$ vector multiplet

$$
W_{i}=\frac{g_{i}}{k+1} \operatorname{Tr} \phi_{i}^{k+1}+\text { lower order }
$$

The precise form of the superpotential is dictated by the fibration data. Adding $N_{0}$ D3 branes, the quiver gauge theory becomes $\prod_{i=0}^{r} U\left(\widehat{N}_{i}\right)$, based on the affine $\widehat{G}$ Dynkin diagram, with

$$
\widehat{N}_{i}=N_{0} d_{i}+N_{i}
$$

for $i \neq 0$ with $d_{i}$ the Dynkin indices. We also set $\widehat{N}_{0}=N_{0}$.

The inequivalent blowups for $\mathcal{N}=1 \mathrm{~A}-\mathrm{D}-\mathrm{E}$ quiver theories are given by the action of the Weyl group. As we will discuss, a Weyl reflection on a node is related to a Seiberg-like duality on the corresponding gauge group. A similar statement applies to the affine case. The duality cascade of [12], for example, corresponds to the affine $\widehat{A}_{1}$ case of $X(k=1, G=$ $\left.\widehat{A}_{1}\right)$. This will be generalized here to the arbitrary affine case. The generalized duality cascade is related to the affine Weyl group, which is the semi-direct product of the Weyl group and translation by the root lattice; the translation is responsible for the cascading reduction of the D3 branes as we flow to the IR.

For the type (ii) case, with compact 4-cycles in addition to the two-cycles, we consider local threefolds which have a toric realizations, as in the examples studied in [18. We can

2 More generally we can consider one $k$ for each simple root of A-D-E, but this can also be obtained, by deformations, from the case we consider. 
then consider wrapping general classes of D3,D5 and D7 branes. In this case, it is more convenient to use the mirror IIA picture of the manifold and branes, as it does not suffer from quantum corrections. Using the appropriate mirror symmetry in the context of branes [19], we write down the corresponding quiver theory, as well as the corresponding Seiberg-like dualities. The dualities involve changes of the classical parameters in the type IIA mirror. We specialize to the Calabi-Yau threefolds involving delPezzo and their transitions. Certain aspects of this case have been noted recently in [20,21].

The organization of this paper is as follows: In section 2 we give an overview of the $\mathcal{N}=1 \mathrm{~A}-\mathrm{D}-\mathrm{E}$ quiver theories and the results we will find for them in this paper. In section 3 we give the description of classical aspects of the A-D-E quiver gauge theories under consideration. In section 4 we discuss some aspects of the quantum dynamics of the gauge couplings and their running. In section 5 we discuss gaugino condensation in the nonaffine $\mathrm{A}-\mathrm{D}-\mathrm{E} \mathcal{N}=1$ quiver theories. In section 6 we consider the geometric engineering of these theories and their large $N$ dual, involving the leading quantum corrections and the geometric realization of gaugino condensates. In section 7 we discuss Seiberg-like dualities for the A-D-E quiver theories anticipated from geometry. In section 8 we discuss the gauge theoretic interpretation of these dualities. In section 9 we consider the gauge theory dynamics of the $A_{2}$ quiver in more detail, as a typical situation where the Seiberg-like duality is relevant. In section 10 we discuss dynamical aspects of the affine quiver theory and its relation to the non-affine case. We also note the connection of RG cascades in this class of theories with affine Weyl reflection. In section 11 we discuss examples of $\mathcal{N}=1$ superconformal A-D-E quiver theories. In section 12 we setup the geometric engineering of the type (ii) local threefolds, as well as dualities predicted by geometry. In section 13 we specialize to a class of examples and illustrate how the gaugino condensation takes place in these chiral theories and what geometric transition they correspond to.

\section{Basic structure of the type (i) $\mathcal{N}=1$ quiver theories and their large $N$ duals}

The class of type (i) theories which we consider are fibrations of a A-D-E twofold geometry over a plane. The corresponding field theory is that of an $\mathcal{N}=2 A-D-E$ or

affine $\widehat{A}-\widehat{D}-\widehat{E}$ quiver theory, deformed to $\mathcal{N}=1$ by superpotential terms $W_{i}\left(\phi_{i}\right)$, with $\phi_{i}$ the adjoint field in the $\mathcal{N}=2 U\left(N_{i}\right)$ vector multiplet. The choice of $W_{i}$ 's are encoded in the fibration data. For simplicity we consider the case where all the superpotentials are polynomials of degree $k+1$. 
The case $X\left(k=1, G=A_{1}\right)$, for example, corresponds to the small resolution of the conifold, in which the $S^{2}$ is blown up. Wrapping $N$ D5 branes on the $S^{2}$ leads to $\mathcal{N}=1$ $U(N)$ pure Yang-Mills. It was argued in [7] that for large $N$, or in the IR, the theory is better described by the geometric conifold transition: $X \rightarrow \widetilde{X}$, where $\widetilde{X}$ is the deformed conifold, with its blown up $S^{3}$ having RR flux. The generalization to $X\left(k, A_{1}\right)$ for arbitrary $k$ was discussed in [13]: the worldvolume theory is $\mathcal{N}=2 U(N)$ gauge theory, broken to $\mathcal{N}=1$ by a superpotential as in (1.1). Geometrically this means that instead of having holomorphic $S^{2}$ 's over the whole plane (corresponding to vev of $\Phi$ ) they only appear at $k$ points. Let us label these $S^{2}$ 's by $S_{p}^{2}$ where $p=1, \ldots, k$. One can distribute the $N$ D5 branes by wrapping them on any of the $S_{p}^{2}$ 's, leading to a Higgsing $U(N) \rightarrow \prod_{p=1}^{k} U\left(M_{p}\right)$. The geometric transition duality of [13] involves $X\left(k, A_{1}\right) \rightarrow \widetilde{X}\left(k, A_{1}\right)$ in which every $S_{p}^{2}$ is blown down and replaced with a blown up $S_{p}^{3}$ having RR flux. The geometric transition duality yields a new field theory duality, in which the original $U(N)$ theory is dual to a $\mathcal{N}=2 U(1)^{k}$ theory, which is broken to $\mathcal{N}=1$ by a particular superpotential (which can be regarded as electric and magnetic FI terms). This duality was shown to be a powerful tool for obtaining exact results about these supersymmetric field theories [13].

The case of $N_{0}$ D3 branes transverse to $X(k=1, G)$, without wrapped D5s, was discussed in [22]. The gauge group is as in (1.2), with all $N_{i}=0$, and these theories flow to $\mathcal{N}=1$ superconformal field theories. These superconformal field theories have a holographic dual description in terms of IIB string theory on $A_{d} S_{5} \times M_{5}(1, G)$ which was discussed in [22], generalizing the work [23] ccorresponding to $G=A_{1}$. We can now add wrapped D5s (sometimes referred to as adding fractional D3 branes), which breaks the conformal invariance. As will be discussed, this theory undergoes a RG cascade generalizing that of [12], which is the case coming from $X\left(k=1, G=A_{1}\right)$.

The geometry of the general $X(k, G)$ and the classical gauge theories associated with arbitrary wrapped D5s, and arbitrary transverse D3s, was obtained in [17]. It was shown there that the basic aspects of the geometry and geometric transition duality matches with what one expects for the field theory in terms of gaugino condensates. One major aim of the present work is to analyze the dynamics of these gauge theories in detail, and verify that the geometry properly predicts the correct gauge theory dynamics. We will see that the associated field theory dualities are geometrically realized via two different possible geometric dual operations:

$$
\begin{aligned}
& (A): S_{i}^{2} \rightarrow \sum_{j} A_{i j} S_{j}^{2} \\
& (B): S_{p}^{2} \rightarrow S_{p}^{3} .
\end{aligned}
$$


The operation $(B)$ is the geometric transition duality, which occurs when a $U(N)$ gauge theory confines, with gaugino condensation. The size of the $S_{p}^{3}$ is related to the gaugino condensate [7, 13].

The operations $(A)$ on the other hand, correspond to Seiberg-type dualities [24] which, from essentially the same viewpoint, was discussed in [3, 4] (see also the related work [25,26]). As we will discuss, these are related to the $G$ Weyl group for $N_{0}=0$ or, for general $N_{0}$, to the $\widehat{G}$ affine Weyl group. Each Seiberg-like duality corresponds to a Weyl reflection about a simple root, with the reflections about each of the simple roots generating the full Weyl group. In particular, all of the $A_{i j}$ in (2.1) are given by Weyl reflections about each simple root $\vec{e}_{i_{0}}$ as

$$
\vec{e}_{i} \rightarrow \vec{e}_{i}^{\prime}=\vec{e}_{i}-\left(\vec{e}_{i} \cdot \vec{e}_{i_{0}}\right) \vec{e}_{i_{0}} \equiv \sum_{j} A_{i j} \vec{e}_{j}
$$

(including the affine root $\vec{e}_{0}$ and its Weyl translation in the $\widehat{G}$ case). The rank of the gauge group is determined by D-brane charge conservation (as in [3, [1]):

$$
\sum N_{i} \vec{e}_{i}=\sum N_{i}^{\prime} \vec{e}_{i}^{\prime}
$$

which implies that

$$
N_{i}^{\prime}=\left(A^{-T}\right)_{i j} N_{j}
$$

The Weyl symmetry $(A)$ acts on the $U\left(N_{i}\right)$ coupling constants and on the superpotentials as

$$
g_{i}^{-2} \rightarrow \sum_{j} A_{i j} g_{j}^{-2}, \quad W_{i}\left(\phi_{i}\right) \rightarrow \sum_{j} A_{i j} W_{j}\left(\phi_{i}\right) .
$$

The action of (2.3) on $g_{i}^{-2}$ follows from the fact that this is identified with the quantum volume of $S_{i}^{2}$. When $S_{i}^{2}$ shrinks the $1 / g_{i}^{2} \rightarrow 0$ (i.e. the theory is strongly coupled) and if we continue it past that it become negative. However we know that another $S^{2}$ has emerged whose volume is $-1 / g_{i}^{2}$ which now is positive. This is the dual gauge theory. From this point of view the duality can be viewed as an attempt to make the $1 / g_{i}^{2}$, s positive. In the field theory, dimensional transmutation can occur, with the running $g_{i}$ written in terms of dynamical scales $\Lambda_{i}$; the action of (2.3) on $g_{i}^{-2}$ then becomes a statement about matching the dynamical scales $\Lambda_{i}$ of the dual theories. The duality is inherited from that duality of the corresponding $\mathcal{N}=2$ theory with $W_{i}=0$, which in the field theory setup was noted in [16] (see also [27]), corresponding to $U\left(N_{c}\right)$ theory with $N_{f}$ flavors getting related 
to $U\left(N_{f}-N_{c}\right)$ with $N_{f}$ flavors. Breaking to $\mathcal{N}=1$ by $W \sim \operatorname{Tr} \phi^{2}$ was considered in [16] and the case of more general $W \sim \operatorname{Tr} \phi^{k+1}$ was considered e.g. in [15] via NS brane constructions.

The two transitions $(A)$ and $(B)$ combine in a beautiful way in the geometric dual description. Geometrically, if we start far from the tip of the cone, the geometry has a description in terms of $S_{i}^{2}$ 's, which change in size as we come closer to the tip of the cone (which is the geometric realization of moving towards the IR). Sometimes an $S^{2}$ shrinks and a dual $S^{2}$ grows, an $(A)$ type transition, which is interpreted as Seiberglike duality. Sometimes an $S^{2}$ shrinks and an $S^{3}$ grows, a $(B)$ type transition, and this corresponds to the occurrence of confinement and gaugino condensation. The nice thing about this picture is that not only can we "derive" Seiberg-like dualities by connecting branes wrapping cycles of Calabi-Yau, as in [3, 4], but in fact we are able to see how they occur in a dynamical sense, i.e. following the RG trajectory and seeing that they become equivalent. This picture works equally well for $G$ as well as for the affine $\widehat{G}$ type quiver theories. The application of duality is particularly striking in the affine case, as one may have to undergo infinitely many applications of duality as we go from the UV to the IR. In particular the RG cascade of [12] corresponds to the $\widehat{A}_{1}$ Weyl group. Upon flowing to the IR, one undergoes a series of $(A)$ type transitions until eventually the theory confines and undergoes the $(B)$ type transition.

Consider the $G=A-D-E$ quiver theories. Using the action (2.3) of the Weyl group on the coupling constants, one can represent the coupling constants as a $r=\operatorname{rank}(G)$ vector $\vec{x}$ such that

$$
\frac{1}{g_{i}^{2}(\vec{x})}=\vec{e}_{i} \cdot \vec{x}>0
$$

The space of $\vec{x}$ satisfying this condition is a $G$ Weyl chamber, a fundamental domain for the action of the Weyl group on $\mathbb{R}^{r} / W$ where $W$ is the Weyl group. The Weyl chamber is a conical wedge, which has $r$ codimension one boundaries, given by $g_{i}^{-2}=0$ for any $i=1 \ldots r$. The RG flow corresponds to moving $\vec{x}$ inside the fundamental domain along a straight line, until it hits a boundary where one of the $1 / g_{i}^{2}=0$. After this, if the ranks of the dual theories are all positive, there is a reflection off the boundary, with incident angle equal to the reflection angle; this corresponds to a Seiberg-like duality. If the rank of the dual theory is not positive, there is no reflection and this is indicative of the (B) type transition in (2.1), corresponding to confinement and gaugino condensation. 
The above picture also applies to the affine quiver theories, with the couplings for the non-affine nodes still labeled by $\vec{x}$ exactly as in (2.4), for $i=1 \ldots r$. The only new feature is the existence of the extra affine node, $i=0$, whose gauge coupling is given by

$$
g_{0}^{-2}(\vec{x})=\frac{1}{g_{s}}-\sum_{i=1}^{r} d_{i} \vec{e}_{i} \cdot \vec{x}
$$

The $d_{i}$ in (2.5) are the Dynkin indices, and the extending simple root is $\vec{e}_{0}=-\sum_{i=1}^{r} d_{i} \vec{e}_{i}$, which can be written as $\sum_{i=0}^{r} d_{i} \vec{e}_{i}=0$ with $d_{0} \equiv 1$. There is now the further restriction on the space of allowed $\vec{x}$ that the RHS of (2.5) is also non-negative. This gives an additional codimension one boundary, cutting the Weyl chamber wedge to a finite sized box; this space of allowed $x$ is the $\widehat{G}$ Coxeter box. It can be viewed as the fundamental domain of the affine Weyl group action on $\frac{\mathbb{R}^{r}}{\widehat{W}}$ where $\widehat{W}$ is the affine Weyl group. Equivalently it can be viewed as the fundamental domain of the Cartan torus by the Weyl group action, $T^{r} / W$, noting that $\widehat{W}=W \ltimes T$ where $T$ is the translation group of the root lattice. Note that a linear combination $\sum_{i=0}^{r} d_{i} g_{i}^{-2}(x)=1 / g_{s}$ is actually independent of $\vec{x}$. This is the gauge coupling of a diagonal $U\left(N_{0}\right)$. Including the theta angles the complex version of this statement is also true: the complex gauge coupling

$$
\tau_{D} \equiv \sum_{i=0}^{r} d_{i} \tau_{i}=\tau_{I I B}
$$

with $\tau_{I I B}$ the IIB string coupling.

A special case of the above discussion for the $\mathcal{N}=1$ affine $\widehat{G}$ quiver theories is the case $N_{i}=0$ wrapped D5s, with $N_{0} \neq 0$ transverse D3s. This case leads to a $\mathcal{N}=$ 1 superconformal field theory with a $r+1$ complex dimensional moduli space of gauge couplings $\tau_{i}$, which are the complexification of the couplings in (2.4) and (2.5). The Weyl reflection dualities maps the theory back to itself, except for changing the coupling constants. This is part of the S-duality group of these theories. The remaining S-duality is the usual $S L(2, \mathbb{Z})$ action on the diagonal gauge coupling (2.6). So a fundamental domain of the moduli space of the $\mathcal{N}=1$ superconformal field theories is given by (the complexification of )(2.4) and (2.5), with $\vec{x}$ in the $\widehat{G}$ Coxeter box, along with the $S L(2, \mathbb{Z})$ fundamental domain for $\tau_{D}$.

This same picture holds for the special case where the deforming $W_{i}\left(\phi_{i}\right)$ vanish, leading to $\mathcal{N}=2$ rather than $\mathcal{N}=1$ superconformal field theories. The Coxeter box structure for the moduli space was found in the related case of D5 branes at a $\mathbb{C}^{2} / \Gamma_{G}$ singularity 
[28,29] in [30]. The moduli space for the $\mathcal{N}=2$ superconformal $\widehat{G}$ theories, setting $W_{i}=0$, was studied in [31] for the $\widehat{A}$-case and generalized to all the $\widehat{A}-\widehat{D}-\widehat{E}$ quiver cases in [18], where the moduli space of couplings was shown to be identified with moduli of flat A-D-E connections on $T^{2}$. The Coxeter box can be identified with the moduli space of flat A-D-E connection on $S^{1}$ and the description of the moduli space along the lines discussed here was noted in [32]. Again, for both the $\mathcal{N}=2$ and the $\mathcal{N}=1$ superconformal theories with $W_{i}\left(\phi_{i}\right) \neq 0$, the S-duality group corresponds to $S L(2, \mathbb{Z})$ action on (2.6), along with the Weyl reflections on the $\tau_{i}$, as in (2.3).

\section{The classical quiver gauge theories}

\section{1. $4 d \mathcal{N}=1 A-D$-E quiver theories}

The class of $\mathcal{N}=1$ quiver gauge theories we consider is a deformation of $\mathcal{N}=2$ quiver gauge theories with gauge group $\prod_{i} U\left(N_{i}\right)$, with $i$ running over the nodes of the quiver diagram, and bi-fundamental hypermultiplets for the linked nodes $i$ and $j$; these hypermultiplets can be written as $\mathcal{N}=1$ chiral superfields $Q_{i j}$ in the $\left(N_{i}, \bar{N}_{j}\right)$ and $Q_{j i}$ in the $\left(\bar{N}_{i}, N_{j}\right)$ of $U\left(N_{i}\right) \times U\left(N_{j}\right)$. The quiver diagrams of interest here are the $G=A, D, E$, or affine $\widehat{G}=\widehat{A}, \widehat{D}, \widehat{E}$, Dynkin diagrams; these are the most general asymptotically free, or conformal respectively, $\mathcal{N}=2$ quiver gauge theories 18 . We consider deformations of these theories to $\mathcal{N}=1$ supersymmetric theories by adding a superpotential for the adjoint fields, $W_{i}\left(\phi_{i}\right)$, so the full tree-level superpotential is

$$
W=\sum_{i}\left[\operatorname{Tr} \sum_{j} s_{i j} Q_{i j} Q_{j i} \phi_{i}-\operatorname{Tr} W_{i}\left(\phi_{i}\right)\right]
$$

where $s_{i j}=-s_{j i}$ is the intersection matrix of nodes $i$ and $j$, which is zero if the nodes are not linked and \pm 1 if they are linked (nothing depends on the choice for the $s_{i j}$ signs). The first term in (3.1) is that of the original undeformed $\mathcal{N}=2$ theory.

In the non-affine case, there is no restriction on $W_{i}\left(\phi_{i}\right)$. In the affine case, however, the geometric engineering of these quiver theories [17] leads to one restriction on the superpotentials:

$$
\sum_{i=0}^{r} d_{i} W_{i}(x)=0
$$

The equations of motion following from (3.1) are

$$
\sum_{j} s_{i j} Q_{i j} Q_{j i}=\partial_{i} W_{i}\left(\phi_{i}\right), \quad \phi_{i} Q_{i j}=Q_{i j} \phi_{j}
$$


for every $Q_{i j}$. The vacua are the solutions of these equations, modulo complexified gauge transformations. We now review the vacuum structure, which was derived in [17]. For the case where the quiver diagram is a non-affine $G=A, D, E$ Dynkin diagram, there are various vacua which are given in terms of the positive roots $\vec{\rho}_{K} \subset \Delta^{+}$of $G$; here $K=1, \ldots, R_{+}$, with $2 R_{+}+r=|G|$, and the positive roots can be expanded in terms of the simple roots $\vec{e}_{i}$ as

$$
\vec{\rho}_{K}=\sum_{i=1}^{r} n_{K}^{i} \vec{e}_{i}
$$

for appropriate $n_{K}^{i} \geq 0$. This corresponds to the fact that the associated geometry has 2-cycles $S_{K}^{2}$ corresponding to the positive roots $\vec{\rho}_{K}$.

For each $\vec{\rho}_{K}$ there are a number of irreducible branches of the supersymmetric theory, given by the roots $x$ the equation

$$
W_{K}^{\prime}(x) \equiv \sum_{i} n_{K}^{i} W_{i}^{\prime}(x)=0
$$

For simplicity we take all $W$ 's to be polynomials of the same degree $k+1$ (the more general case can also be constructed geometrically [17]). Then, for each positive root $\vec{\rho}_{K}$, the above equation has $k$ roots, which we label as $x=a_{(p, K)}$, with $p=1, \ldots, k$ and $K=1 \ldots R_{+}$. There is a susy vacuum for every choice of $M_{(p, K)} \geq 0$ such that

$$
N_{i}=\sum_{K=1}^{R_{+}} \sum_{p=1}^{k} M_{(p, K)} n_{K}^{i} .
$$

In these vacua $\phi_{i}$ has $n_{K}^{i} M_{(p, K)}$ eigenvalues given by the root $a_{(p, K)}$ and the gauge group is Higgsed as

$$
\prod_{i=1}^{r} U\left(N_{i}\right) \rightarrow \prod_{K=1}^{R_{+}} \prod_{p=1}^{k} U\left(M_{(p, K)}\right) .
$$

For the case of affine quiver diagrams, the vacua are similarly labeled by the positive affine roots [17]. We will consider the cases where there are no pure 3-brane branches (this is the analogue of the Coulomb branch of the $\mathcal{N}=4$ ). In this case the Higgs branches are also labeled by the positive roots of affine A-D-E, which are described as follows: Recall that the highest root of $G$ is $\psi=\sum_{j=1}^{r} d_{j} e_{j}$, with $e_{j}$ the simple roots; the extending affine root $e_{0}$ is $e_{0} \equiv-\psi$, so $\sum_{i=0}^{r} d_{i} e_{i}=0$, with $d_{0} \equiv 1$. The extended Cartan matrix is $C_{i j}=e_{i} \cdot e_{j}$ for all $i, j=0, \ldots, r$. For affine Lie algebras one replaces $e_{i} \rightarrow \widehat{e}_{i}=\left(e_{i}, 0\right)$ for $i=1, \ldots, r$ and $e_{0} \rightarrow \widehat{e}_{0}=(-\psi, 1)$. Note that $\sum_{i=0}^{r} d_{i} \widehat{e}_{i}=\delta$, with $\delta=(0,1)$ which we 
identify as the D3 brane charge direction (called the 'imaginary direction' for the affine algebra). The positive roots of the affine algebra are given by

$$
\widehat{\vec{\rho}}_{\widehat{K}}: \quad\left(\Delta, n^{+}\right),\left(\Delta^{+}, 0\right)
$$

where $n^{+}$is a positive integer and $\Delta$ denotes all roots. Each such vector can be written as positive combination of positive affine roots:

$$
\widehat{\vec{\rho}}_{\widehat{K}}=\sum_{i=0}^{r} n_{\widehat{K}}^{i} \widehat{e}_{i}
$$

For each such root, consider its projection to the root lattice which is either a positive root or its negative, given by $\pm \sum_{i=1}^{r} n_{K}^{i} e_{i}$ as $K=1, \ldots, R_{+}$. For each such branch we consider solutions to

$$
W^{\prime}\left(\widehat{\vec{\rho}}_{\widehat{K}}\right)= \pm \sum_{i} W_{i}^{\prime}(x) n_{K}^{i}=0
$$

which is exactly the equation we considered in the non-affine case (the possible minus sign does not affect the solutions to the above equation). There are $k$ solutions for each branch,

which we label with $(p, \widehat{K})$. Choose non-negative integers $M_{(p, \widehat{K})}$ which label how many of each irreducible branch we choose. These should satisfy

$$
\sum_{\widehat{K}} M_{(p, \widehat{K})} n_{\widehat{K}}^{i}=\hat{N}_{i}
$$

In this branch the gauge group is Higgsed to

$$
\prod_{i=0}^{r} U\left(\widehat{N}_{i}\right) \rightarrow \prod_{\widehat{K}} \prod_{p=1}^{k} U\left(M_{(p, \widehat{K})}\right) .
$$

\section{Aspects of the quantum dynamics: gauge couplings and their running}

In this section we discuss some aspects of the quantum dynamics of the gauge couplings and their running as a function of scale. We will first consider the underlying $\mathcal{N}=2$ quiver theory and then we will discuss aspects of the $\mathcal{N}=1$ deformed theory by adding superpotential terms. 


\section{1. $\mathcal{N}=2$ quiver theories}

First let us ignore the superpotentials $W_{i}\left(\phi_{i}\right)$ so we have an $\mathcal{N}=2$ quiver theory. This is also a good approximation for the dynamics of the $\mathcal{N}=1$ quiver theory for energy scales large enough compared to the superpotential deformations (i.e. for scales $\mu$ large compared to the adjoint mass $\left.W_{i}^{\prime \prime}(\mu)\right)$.

The $N=2$ exact beta function for the coupling $\tau_{i} \equiv \frac{\theta_{i}}{2 \pi}+4 \pi i g_{i}^{-2}$ of $U\left(N_{i}\right)$ is

$$
\beta_{i} \equiv-2 \pi i \beta\left(\tau_{i}\right)=\sum_{j} C_{i j} N_{j}
$$

with $C_{i j}=2 \delta_{i j}-\left|s_{i j}\right|=\vec{e}_{i} \cdot \vec{e}_{j}$ the Cartan matrix of the A-D-E diagram, or the extended Cartan matrix of the affine $\widehat{A}-\widehat{D}-\widehat{E}$ diagram. The sign of $\beta_{i}$ in (4.1) is chosen so that the theory is asymptotically free if (4.1) gives $\beta_{i}>0$. Note that this can be conveniently summarized by a vector $\vec{\beta}$ whose projection on $\vec{e}_{i}$ gives $\beta_{i}$

$$
\beta_{i}=\vec{e}_{i} \cdot \vec{\beta} \quad \text { with } \quad \vec{\beta}=\vec{N} \equiv \sum_{i} N_{i} \vec{e}_{i}
$$

In the affine $\widehat{G}$ case we include the affine node $i=0$ in (4.2). In the affine case, since $\sum_{i=0}^{r} d_{i} \vec{e}_{i}=0$, the $\widehat{G}$ affine quiver theory with $\hat{N}_{i}=N_{0} d_{i}$ has $\vec{\beta}=0$; it's an $\mathcal{N}=2$ superconformal field theory for any $N_{0}$. This corresponds to $N_{0}$ D3 branes and no wrapped D5 branes. There are $r+1$ complex moduli, given by the $U\left(\hat{N}_{i}\right)$ gauge couplings $\tau_{i}$ for $i=0, \ldots, r$. More generally, for any $\hat{N}_{i}$, the beta functions (4.1) are invariant under the shift

$$
\hat{N}_{i} \rightarrow \hat{N}_{i}+N_{0} d_{i},
$$

for any $N_{0}$. Also, for any $\hat{N}_{i}$, the beta function for the coupling

$$
\tau_{D} \equiv \sum_{i=0}^{r} d_{i} \tau_{i}
$$

of a diagonally embedded $U(N)$ vanishes, as $\sum_{i} \beta_{i} d_{i}=0$.

In the construction of the affine $\widehat{G}$ quiver theories [28] via D3 branes at $G$ type ALE singularities, $\tau_{D}$ is the IIB string coupling, while the other $\tau_{i}$ are given by the orbifold blowing up modes coming from the twisted sector NS or RR fields, as in [32]. Thus $\tau_{D}$ must have the $S L(2, \mathbb{Z})$ S-duality of IIB string theory. The other independent $\tau_{i}$ also exhibit S-dualities, which correspond to $G$ Weyl reflections. As already mentioned, this shows that the $r+1$ complex dimensional moduli space of the $\widehat{G}$ quiver $\mathcal{N}=2$ superconformal theories consists of the $S L(2, \mathbb{Z})$ fundamental domain for $\tau_{D}$, along with the complexification of the $\widehat{G}$ Coxeter box for the remaining linear combinations of the couplings $\tau_{i}$ of each $U\left(\hat{N}_{i}\right)$ gauge group factor. 


\subsection{The $\mathcal{N}=1$ quiver theories}

Now consider the $\mathcal{N}=1 \mathrm{~A}-\mathrm{D}-\mathrm{E}$ quiver theories, with superpotential as in (3.1), with $W_{i}\left(\phi_{i}\right)$ as in (1.1): $W_{i} \sim \operatorname{Tr} \phi_{i}^{k+1}+$ lower order. For $k=1$ the $\phi_{i}$ are massive and can be integrated out; for $k>1$ the $\phi_{i}$ should be kept (unless one adds the generic lower order terms in (1.1), in which case $\phi_{i}$ is again massive and can be integrated out at low energies). Note that, for $k>1$, the deformations of the superpotential appear to be irrelevant $(k=2$ is marginally irrelevant), and thus divergent in the UV limit. In the UV one needs a cutoff to define the theory, but the IR aspects we will discuss are universal and independent of the cutoff. The deforming operators are actually "dangerously irrelevant," much as in [33], in that they get large anomalous dimensions and they control the IR dynamics.

The $\mathcal{N}=2 \rightarrow \mathcal{N}=1$ superpotential deformation $W_{i}\left(\phi_{i}\right)$ does not change the (1-loop exact) holomorphic beta functions, so they are the same as in (4.1):

$$
\beta_{i}=\sum_{j} C_{i j} N_{j}=\vec{e}_{i} \cdot \vec{N}, \quad \text { which gives } \quad e^{2 \pi i \tau_{i}(\mu)}=\left(\frac{\Lambda_{i}}{\mu}\right)^{\beta_{i}} .
$$

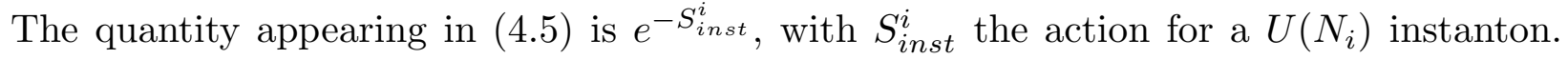
The gauge coupling running (4.5) and scales $\Lambda_{i}$ apply above the mass scale $\Delta \sim W^{\prime \prime}$ where $\phi_{i}$ gets a mass (this occurs for $k=1$ or, for higher $k$, if $W_{i}^{\prime}(x)$ has no coinciding roots). Below the mass scale $\Delta$, the $\phi_{i}$ can be integrated out and the holomorphic beta functions are instead

$$
\beta_{i}^{\text {low }} \equiv-2 \pi i \beta\left(\tau_{i}\right)=N_{i}+\sum_{j} C_{i j} N_{j} .
$$

Matching the running coupling $g_{i}$ at the scale $\Delta$ gives that the low-energy theory has dynamical scale $\Lambda_{i}^{\text {low }}$ given by $\left(\Lambda_{i}^{\text {low }}\right)^{\beta_{i}^{\text {low }}}=\Delta^{N_{i}} \Lambda_{i}^{\beta_{i}}$. The more general matching relations, associated with the different Higgsing branches, will be discussed in detail the following section.

We refer to the above 1-loop beta functions as the "holomorphic beta functions" since, as usual, they exactly give the running of the coefficient of the $U\left(N_{i}\right)$ gauge kinetic term, when the superpotentials are written in terms of the holomorphic (bare) quantities. Also of interest are the "physical beta functions," which are the ones of relevance for analyzing RG flows and determining the existence of RG fixed points. The physical beta functions can be written in terms of the anomalous dimensions [34, which for our theories yields

$$
\begin{aligned}
\beta_{i}^{\text {phys }} \equiv-2 \pi i \beta^{p h y s}\left(\tau_{i}\right) & =3 N_{i}-N_{i}\left(1-\gamma\left(\phi_{i}\right)\right)-\sum_{j \neq i}\left|\vec{e}_{i} \cdot \vec{e}_{j}\right| N_{j}\left(1-\gamma\left(Q_{i j}\right)\right) \\
& =\left(1+\frac{1}{2} \gamma\left(\phi_{i}\right)\right) \sum_{j} C_{i j} N_{j}+\frac{1}{2} \sum_{j \neq i}\left|\vec{e}_{i} \cdot \vec{e}_{j}\right| N_{j} \beta\left(\lambda_{i j}\right),
\end{aligned}
$$


where $\lambda_{i j}$ is the coefficient of $Q_{j i} \Phi_{i} Q_{i j}$ in the superpotential (note that this can be scaled to one, by rescaling $Q$ 's). Here $\gamma\left(\phi_{i}\right)$ is the anomalous dimension of $\phi_{i}$ and we define

$$
\beta\left(\lambda_{i j}\right) \equiv \gamma\left(\phi_{i}\right)+2 \gamma\left(Q_{i j}\right)
$$

which is (proportional to) the beta function for the $\lambda_{i j}$. The expression (4.7) is essentially the NSVZ beta function, though without the denominator factor of [34]; this is because we are using the holomorphic gauge kinetic terms, $\sim \int d^{2} \theta \tau W_{\alpha} W^{\alpha}$, and the canonical matter kinetic terms.

The beta function (4.7) applies above the possible scale $\Delta$ where $\phi_{i}$ could be integrated out. Below such a scale, the exact beta function is

$$
\begin{aligned}
\beta_{i} & =3 N_{i}-\sum_{j \neq i}\left|\vec{e}_{i} \cdot \vec{e}_{j}\right| N_{j}\left(1-\gamma\left(Q_{i j}\right)\right) \\
& =\frac{3}{2} \sum_{j} C_{i j} N_{j}+\sum_{j \neq i}\left|\vec{e}_{i} \cdot \vec{e}_{j}\right| \widehat{\gamma}\left(Q_{i j}\right),
\end{aligned}
$$

where we define $\widehat{\gamma}\left(Q_{i j}\right)=\gamma\left(Q_{i j}\right)+\frac{1}{2}$. Integrating out $\phi_{i}$ induces a quartic superpotential for the $Q_{i j}$, which would be marginal if $\gamma\left(Q_{i j}\right)=-\frac{1}{2}$, corresponding to $\widehat{\gamma}\left(Q_{i j}\right)=0$. Indeed, in this case the beta functions (4.9) all vanish for the affine $\widehat{G}$ quiver theories with $N_{i}=N_{0} d_{i}$

\section{Gaugino condensation in the non-affine $\mathcal{N}=1$ quiver theories}

Let us now consider the dynamics of the $\mathcal{N}=1$ quiver theory taking into account the fact that at scales lower than the relevant scales for the superpotentials $W_{i}$ the theory gets higgsed to various branches. For simplicity let us assume that all the $W_{i}$ 's become relevant at the scale $\Delta$. Thus for scales $\mu>>\Delta$ we effectively have an $\mathcal{N}=2$ quiver theory with the running of the coupling constants we have noted. Let us assume that at the scale $\Delta$ all these couplings are still small, i.e. $1 / g_{i}^{2}(\Delta)>>1$, so that the classical analysis of the branches is reliable. For scales below $\Delta$ the superpotential becomes relevant and the theory is Higgsed (for generic $W_{i}$ to a product of pure $\mathcal{N}=1$ theories $\prod_{p=1}^{k} \prod_{K=1}^{R_{+}} U\left(M_{(p, K)}\right)$ with some additional massive fields. The $S U\left(M_{(p, K)}\right)$ factor in (3.6) gets a mass gap, with gaugino condensation and confinement. 
The naive low-energy superpotential associated with the $\prod S U\left(M_{p, K}\right)$ gaugino condensations can be written as

$$
W_{\text {g.c. }}=\sum_{p=1}^{k} \sum_{K=1}^{R_{+}} S_{(p, K)}\left(\log \left(\frac{\Lambda_{(p, K)}^{3 M_{(p) K}}}{S_{(p, K)}^{M_{(p, K)}}}\right)+M_{(p, K)}\right)
$$

where the $\Lambda_{(p, K)}$ are the scales of the low energy $U\left(M_{(p, K)}\right)$ gauge groups as found via naive threshold matching, which we will discuss in what follows. $S_{(p, K)}$ is the $S U\left(M_{(p, K)}\right)$ glueball field $S_{(p, K)} \sim \operatorname{Tr}_{S U\left(M_{(p, K)}\right)} W_{\alpha} W^{\alpha}$, whose expectation value is the $S U\left(M_{(p, K)}\right)$ gaugino condensate. The $S_{(p, K)}$ in (5.1) are massive and can be integrated out.

In general, the naive gaugino condensation superpotential is only a leading approximation to a more non-trivial exact result. This is seen, among other examples, in the analysis of [13], where the geometric transition duality emerged as a powerful tool to obtain the exact superpotential. Though $W_{\text {g.c. }}$ is not exact, it does exactly give the non-trivial monodromies of the superpotential. Moreover it should be a good approximation in the case where the $\mathcal{N}=2$ gauge couplings are weak at the scale $\Delta$ where the Higgsing takes place. The coupling constant of the $U\left(M_{(p, K)}\right)$ theory at the scale $\Delta$ where Higgsing takes place satisfies

$$
g_{(p, K)}^{-2}(\Delta)=\sum_{i} n_{K}^{i} g_{i}^{-2}(\Delta)
$$

We still need to match the running gauge couplings $g_{(p, K)}$ across the thresholds of various massive matter fields in order to relate the low energy scales $\Lambda_{(p, K)}$ to the high energy scales $\Lambda_{i}$ of the original quiver theory. This is what we will now do.

As discussed earlier, the possible eigenvalues of the adjoints $\phi_{i}$ are the solutions $a_{K}^{p}$, with $p=1 \ldots k$ and $K=1 \ldots R_{+}$, of (3.4). E.g. for the case $k=1$, with $W_{i}=\frac{1}{2} m_{i} \operatorname{Tr} \phi_{i}^{2}$, we have

$$
a_{K}=\frac{\sum_{i} n_{K}^{i} m_{i} a_{i}}{\sum_{i} n_{K}^{i} m_{i}}
$$

In general, $\phi_{i}$ can have $M_{(p, K)} n_{K}^{i}$ eigenvalues equal to $a_{(p, K)}$, with $N_{i}=\sum_{(p, K)} n_{K}^{i} M_{(p, K)}$,

$$
\phi_{i} \rightarrow \oplus_{(p, K)} a_{(p, K)}\left(1_{M_{(p, K)}}\right)^{n_{K}^{i}}
$$

which breaks

$$
U\left(N_{i}\right) \rightarrow \prod_{p=1}^{k} \prod_{K=1}^{R_{+}} U\left(M_{(p, K)}\right)_{i}^{n_{K}^{i}}
$$


Under this breaking the $Q_{i j}$ decompose as

$$
Q_{i j} \rightarrow \oplus_{(p, K)} \oplus_{(q, L)}\left(M_{(p, K)}, \bar{M}_{(q, L)}\right)^{n_{K}^{i} n_{L}^{j}},
$$

with the bifundamental in (5.5) of mass $a_{(p, K)}-a_{(q, L)}$.

There is additional Higgsing, besides that of (5.3), due to non-zero expectation values of components of the $Q_{i j}$; this Higgsing breaks

$$
\prod_{i} U\left(M_{(p, K)}\right)_{i}^{n_{K}^{i}} \rightarrow U\left(M_{(p, K)}\right)
$$

which is a diagonally embedded subgroup. The $Q_{i j}$ expectation values can be seen by plugging into the equations of motion (3.2),

$$
\sum_{j} s_{i j} Q_{i j} Q_{j i}=W_{i}^{\prime}\left(\phi_{i}\right)
$$

which we should evaluate for the above eigenvalues $a_{(p, K)}$ of $\phi_{i}$. In the end, the unbroken gauge group is

$$
\prod_{i=1}^{r} U\left(N_{i}\right) \rightarrow \prod_{p=1}^{k} \prod_{K=1}^{R_{+}} U\left(M_{(p, K)}\right), \quad \text { with } \quad N_{i}=\sum_{p=1}^{k} \sum_{K=1}^{R_{+}} n_{K}^{i} M_{(p, K)} .
$$

The naive superpotential (5.1) arises from gaugino condensation in the unbroken gauge group factors of (5.8).

The original high energy $U\left(N_{i}\right)$ theory, with its adjoint included, has beta functions as in (4.2): $\beta_{i}=\vec{e}_{i} \cdot \vec{N}$, with $\vec{N} \equiv \sum_{i} N_{i} \vec{e}_{i}$. Using (5.8) we can also express these in terms of the ranks $M_{(p, K)}$ of the low-energy gauge group:

$$
\beta_{i}=\vec{e}_{i} \cdot \vec{N}=\vec{e}_{i} \cdot \vec{M} \quad \text { where } \quad \vec{M} \equiv \sum_{p=1}^{k} \sum_{K=1}^{R_{+}} M_{(p, K)} \vec{\rho}_{K} .
$$

Thus we can write the $U\left(N_{i}\right)$ instanton factors, in terms of the dynamical scales $\Lambda_{i}$ of the original high energy theory, as in (4.5):

$$
\Lambda_{i}^{\sum_{j} C_{i j} N_{j}}=\Lambda_{i}^{\vec{N} \cdot \vec{e}_{i}}=\Lambda_{i}^{\vec{M} \cdot \vec{e}_{i}}
$$


We determine the scales $\Lambda_{(p, K)}$ of the low energy $U\left(M_{(p, K)}\right)$ theory in (5.8) by naive threshold matching relations at the scales of all massive $U\left(M_{(p, K)}\right)$ matter and W-boson fields. The result we thus obtain is

$$
\Lambda_{(p, K)}^{3 M_{(p, K)}}=\left[W_{K}^{\prime \prime}\left(a_{(p, K)}\right)\right]^{M_{(p, K)}} \prod_{(q, L) \neq(p, K)}\left(a_{(p, K)}-a_{(q, L)}\right)^{-\vec{\rho}_{K} \cdot \vec{\rho}_{L} M_{(q, L)}} \prod_{i=1}^{r}\left(\Lambda_{i}^{\vec{M} \cdot \vec{e}_{i}}\right)^{n_{K}^{i}}
$$

where we define $W_{K}^{\prime \prime}\left(a_{(p, K)}\right) \equiv \sum_{i=1}^{r} n_{K}^{i} W_{i}^{\prime \prime}\left(a_{(p, K)}\right)$. Note that the RHS of (5.11) properly has mass dimension $M_{(p, K)}\left(1+\vec{\rho}_{K} \cdot \vec{\rho}_{K}\right)=3 M_{(p, K)}$ (since all positive roots of the simply laced $A D E$ satisfy $\left.\vec{\rho}_{K} \cdot \vec{\rho}_{K}=\sum_{i, j=1}^{r} n_{K}^{i} n_{K}^{j} C_{i j}=2\right)$.

To see how (5.11) is obtained, write the exponent of $\left(a_{(p, K)}-a_{(q, L)}\right)$ in (5.11) as $M_{(q, L)} \sum_{i, j=1}^{r} n_{K}^{i} n_{L}^{i} C_{i j}$; the terms involving $C_{i i}=2$ are associated with $W$ boson threshold matching, whereas the $C_{i j}=-1$ terms are associated with threshold matching for matter fields coming from components of $Q_{i j}$. The products of $\Lambda_{i}^{\vec{M} \cdot e_{i}}$, with exponent $n_{K}^{i}$, appearing $\Lambda_{i}$ in (5.11) results from the fact that $U\left(M_{(p, K)}\right)$ arises as the diagonal subgroup, as in (5.6), so the $U\left(M_{(p, K)}\right)$ gauge coupling is

$$
g_{K}^{-2}=\sum_{i} n_{K}^{i} g_{i}^{-2}
$$

at the scale of the Higgsing (5.6), and using (4.5).

We can now plug (5.11) into (5.1) to get the final expression

$$
\begin{aligned}
W_{g . c} & =\sum_{(p, K)} S_{(p, K)}\left(M_{(p, K)}+M_{(p, K)} \log \left(\frac{m_{(p, K)} \prod_{i} \Lambda_{i}^{n_{K}^{i} n_{K}^{j} C_{i j}}}{S_{(p, K)}}\right)\right) \\
& +\sum_{(p, K)} \sum_{(q, L) \neq(p, K)} \sum_{i, j=1}^{r} S_{(p, K)} M_{(q, L)} n_{L}^{i} n_{K}^{j} C_{i j} \log \left(\frac{\Lambda_{i}}{a_{(p, K)}-a_{(q, L)}}\right) .
\end{aligned}
$$

We stress again that this is only an approximation, valid in the regime where the gauge couplings are weak at the scale determined by the superpotentials. Nevertheless, the non-trivial monodromies of (5.13) are expected to be exact, as the additional quantum corrections are single valued.

\section{Geometric Construction}

In this section we will study the geometric realization of the $\mathcal{N}=1 \mathrm{~A}-\mathrm{D}-\mathrm{E}$ quiver theories, and connect with the field theoretic analysis of these theories presented in the 
previous sections. The geometric description allows the formulation of large $\mathrm{N}$ duals via transitions of the form $S^{2} \rightarrow S^{3}$, which we interpret as the field theory developing gaugino condensates. The dynamics of the gauge theory can be mapped to geometric language in a beautiful way. In particular, we show that the running of the gauge couplings is imposed upon us by the log divergences in the periods of the holomorphic three form on non-compact 3-cycles. The superpotential obtained in (5.13), from naive integrating in, is shown to be the leading order approximation in a weak coupling expansion of the exact superpotential given in terms of geometric periods. This leading order approximation can be obtained from the geometry via a monodromy analysis in the form of the Picard-Lefschetz formula.

\subsection{Review of Geometric Engineering of $\mathcal{N}=1 A-D-E$ Quiver theories}

The geometric engineering of $\mathcal{N}=1 \mathrm{~A}-\mathrm{D}-\mathrm{E}$ quiver theories is done in two steps. The first is to consider Type IIB on an ALE space with a blown up A-D-E singularity. Wrapping D5-branes around different non-trivial 2-cycles will give rise to $\mathcal{N}=2$ gauge theories on the world volume. Likewise, adding D3-branes transverse to the ALE space will give

$\mathcal{N}=2$ affine A-D-E quiver theories on their worldvolumes. The second step is to realize that these ALE spaces can also be made nonsingular by adding relevant deformations. These deformations can then vary over the complex plane transverse to the ALE space, D3 and D5 branes. This fibration induces a superpotential in the theories, breaking the supersymmetry down to $\mathcal{N}=1$.

\section{$A-D$-E singularities in dimension 2}

Blown down singular ALE spaces can be viewed as hypersufaces $f(x, y, z)=0$ of $\mathbf{C}^{3}$ :

$$
\begin{array}{ll}
G=A_{r}: & f=x^{2}+y^{2}+z^{r+1} \\
G=D_{r}: & f=x^{2}+y^{2} z+z^{r-1} \\
G=E_{6}: & f=x^{2}+y^{3}+z^{4} \\
G=E_{7}: & f=x^{2}+y^{3}+y z^{3} \\
G=E_{8}: & f=x^{2}+y^{3}+z^{5}
\end{array}
$$

These spaces can be made smooth by adding relevant deformations of the form,

$$
\sum_{i=1}^{r} P_{c_{i}(G)}\left(t_{1}, \ldots, t_{r}\right) R_{C_{2}(G)-c_{i}(G)}(y, z),
$$


where the subscripts are the degrees of the polynomials under the scaling where $t_{i}$ 's have degree one and $f(x, y, z)$ has degree $C_{2}(G)$, the dual Coxeter number of $\mathrm{G}\left(c_{i}(G)\right.$ are the degrees of the Casimirs of $G$ ). Notice that there are $r=\operatorname{rank}(G)$ deformation parameters $t_{i}$ 's. For generic $t_{i}$ 's, there are $r$ independent classes of non-vanishing $S^{2}$ 's and their intersection can be chosen to correspond to the G Dynkin diagram. The holomorphic volumes of the $S_{i}^{2}$ 's are denoted by,

$$
\alpha_{i}=\int_{S_{i}^{2}} \frac{d y d z}{x} \quad \text { for } \quad i=1, \ldots, r
$$

The $\alpha_{i}$ are in 1-1 correspondence with the simple roots of $\mathrm{G}$, and are linearly related to the $t_{i}$.

The deformed ALE space is simple to write in the $A$ and $D$ cases, namely,

$$
\begin{gathered}
A_{r} \quad: \quad x^{2}+y^{2}+\prod_{i=1}^{r+1}\left(z+t_{i}\right) \quad \sum_{i=1}^{r+1} t_{i}=0 \\
D_{r} \quad: \quad x^{2}+y^{2} z+\frac{\prod_{i=1}^{r}\left(z+t_{i}^{2}\right)-\prod_{i=1}^{r} t_{i}^{2}}{z}+2 \prod_{i=1}^{r} t_{i} y
\end{gathered}
$$

and the holomorphic volumes are given by,

$$
\begin{gathered}
A_{r}: \quad \alpha_{i}=t_{i}-t_{i+1} \quad i=1, \ldots, r \\
D_{r}: \quad \alpha_{i}=t_{i}-t_{i+1} \quad i=1, \ldots, r-1 \quad \text { and } \quad \alpha_{r}=t_{r-1}+t_{r}
\end{gathered}
$$

The corresponding equations for $E_{6}, E_{7}$ and $E_{8}$ deformations in terms of $t$ 's (which are chosen to be linearly related to $\alpha$ 's) are more complicated and we refer the reader to [35].

\section{Fibration}

We want to obtain a Calabi-Yau 3-fold by fibering the ALE space described above over a complex plane whose coordinate we denote by $t$. This fibration is implemented by allowing the $t_{i}$ 's to be polynomials in $t$. Therefore, the holomorphic volumes $\alpha_{i}$ will also be functions of $t$. Wrapping $N_{i}$ D5 branes around the $S_{i}^{2}$ fiber, but with world volume transverse to the t-plane, will induce a classical superpotential in the gauge theory satisfying,

$$
W_{i}^{\prime}(t)=\alpha_{i}(t)
$$

where $t$ corresponds to $\left\langle\Phi_{i}\right\rangle$, the expectation value of the adjoint of the $U\left(N_{i}\right)$. 
Notice that without the superpotentials, i.e., with a trivial fibration, the normal bundle over each $S^{2}$ is $\mathcal{O}(-2) \oplus \mathcal{O}(0)$. However, with the introduction of superpotentials, the geometry will have points where a given cycle can have zero holomorphic volume. These points, which are singular in the geometry, can be blown up, giving rise to $S^{2}$ 's with normal bundle $\mathcal{O}(-1) \oplus \mathcal{O}(-1)$. If the degree of $W_{i}^{\prime}(t)$ is $k$, there will be $k$ points in the t-plane for each positive root $\vec{\rho}_{K}$ of $\mathrm{G}$ where the holomorphic volume vanishes:

$$
\alpha\left(\vec{\rho}_{K}\right)=\sum_{i=1}^{r} n_{K}^{i} \alpha_{i}(t)=\sum_{i=1}^{r} n_{K}^{i} W_{i}^{\prime}(t)=0 .
$$

These are the supersymmetric vacua corresponding to the Higgsing (3.6) where we wrap $M_{p, K}$ D5 branes around the cycle at the $p$-th solution of (6.5). Let us rewrite (3.6),

$$
\prod_{i} U\left(N_{i}\right) \rightarrow \prod_{K=1}^{R_{+}} \prod_{p=1}^{k} U\left(M_{(p, K)}\right) .
$$

Clearly, the charge conservation condition is (3.5),

$$
N_{i}=\sum_{K=1}^{R_{+}} \sum_{p=1}^{k} M_{(p, K)} n_{K}^{i} .
$$

\subsection{Large $N$ duality}

In the IR limit of the gauge theory we are left with pure $\mathcal{N}=1 \mathrm{SYM}$ with gauge group $\prod_{K=1}^{R_{+}} \prod_{p=1}^{k} U\left(M_{(p, K)}\right)$. This theory is expected to have gaugino condensation in each factor of the gauge group, as discussed in section 5. As in [12, 36, [7, 13], the proposal is that the geometry realizes this process by geometric transitions of the form $S_{(p, K)}^{2} \rightarrow S_{(p, K)}^{3}$. It is important to notice that all these are conifold-like transitions since the $S_{(p, K)}^{2}$ 's being blown down have normal bundle $\mathcal{O}(-1) \oplus \mathcal{O}(-1)$.

The number of singular points after blowing down all $S_{(p, K)}^{2}$ 's is $k R_{+}$, where $k$ is the degree of $W_{i}^{\prime}(t)$ 's. The large $\mathrm{N}$ dual is therefore achieved by deforming the complex structure of the singular Calabi-Yau 3-fold,

$$
x^{2}+F\left(y, z, t_{1}(t), \ldots, t_{r}(t)\right)=0
$$

by normalizable deformations (including the log normalizable). These normalizable deformations correspond to dynamical fields, as opposed to fixed parameters [37]. The dynamical fields which they correspond to are precisely the $S U\left(M_{(p, K)}\right)$ glueball fields 
$S_{(p, K)} \sim \operatorname{Tr}_{S U\left(M_{(p, K)}\right)} W_{\alpha} W^{\alpha}$. In [17], it was shown that the total number of these normalizable deformations is exactly $k R_{+}$, the expected number of $S^{3}$ 's. This matches the natural idea that the $k R_{+}$gaugino condensates are independent, dynamical, and control the sizes of the $S^{3}$, parameterizing the deformation of the geometry.

The normalizable deformations can be easily found by noting that (6.6) has the form

$$
f(x, y, z)+a t^{k C_{2}(G)}+\ldots=0
$$

Charges can be assigned to $x, y, z$, and $t$ such that the above equation has charge 1 . In particular, $t$ will always have charge $1 / k C_{2}(G)$. Thinking about (6.7) as the superpotential of a Landau-Ginzburg theory, the central charge is given by,

$\hat{c}=(1-2 Q(x))+(1-2 Q(y))+(1-2 Q(z))+(1-2 Q(t))=\frac{2}{k C_{2}(G)}\left(k\left(C_{2}(G)-1\right)-1\right)$.

The normalizable deformations are those monomials $t^{\beta} y^{\delta} z^{\gamma}$ with charge $Q\left(t^{\beta} y^{\delta} z^{\gamma}\right)<\frac{\hat{c}}{2}$; we also include the log normalizable deformations, with charge $Q\left(t^{\beta} y^{\delta} z^{\gamma}\right)=\frac{\hat{c}}{2}$ [37].

\section{Periods and Superpotential}

The geometry after the deformation is smooth and contains $k R_{+}$non-trivial $S^{3}$ 's. These 3-cycles form a natural basis for $A_{(p, K)}$ cycles in the Calabi-Yau geometry and we define $k R_{+}$non compact cycles $B_{(p, K)}$ dual to the $A_{(p, K)}$ 's producing a symplectic pairing. An important role in the sequel is played by the periods of the holomorphic three form $\Omega$ over $A_{(p, K)}$ 's and $B_{(p, K)}$ 's. We denote the periods,

$$
\int_{A_{(p, K)}} \Omega \equiv S_{(p, K)} \quad \int_{B_{(p, K)}}^{\Lambda_{0}} \Omega \equiv \Pi_{(p, K)}=\frac{\partial \mathcal{F}}{\partial S_{(p, K)}}
$$

where $\Lambda_{0}$ is a cutoff needed to regulate the divergent $B_{(p, K)}$ integrals. The $k R_{+}$periods $S_{(p, K)}$ are determined by (6.9) in terms of the coefficients of the $k R_{+}$normalizable deformations. One can then invert these relations, to write the coefficients of the normalizable deformations in terms of the $S_{(p, K)}$.

After the transition the $\mathrm{D}$ branes have disappeared and have been replaced by fluxes on the $S^{3}$ 's of a suitable 3 -form $H$. This leads to a superpotential [38, 39],

$$
W=\int H \wedge \Omega=\sum_{p=1}^{k} \sum_{K=1}^{R_{+}}\left(\int_{A_{(p, K)}} H \int_{B_{(p, K)}} \Omega-\int_{B_{(p, K)}} H \int_{A_{(p, K)}} \Omega\right) .
$$


This thus gives for the full effective superpotential

$$
-\frac{1}{2 \pi i} W=\sum_{p=1}^{k} \sum_{K=1}^{R_{+}}\left(M_{(p, K)} \Pi_{(p, K)}+\frac{\alpha_{K}}{2 \pi i} S_{(p, K)}\right)
$$

where $\alpha_{K}$ 's are related to the bare coupling constants of the original $U\left(N_{i}\right)$ 's with $i=$ $1, \ldots, r$ of the quiver theory. The precise correspondence will be given below when we show that the logarithmic dependence on $\Lambda_{0}$ of $\Pi$ 's can be absorbed in the $\alpha$ 's, rendering the superpotential finite (up to irrelevant constant terms) as we send the cut off $\Lambda_{0}$ to infinity.

\subsection{Dynamics of the theory}

It was shown in 13. for the $G=A_{1}$ quiver theory case that (6.11) is the exact effective superpotential of the $X\left(k, A_{1}\right)$ theory, and that the superpotential obtained from naive integrating in is the leading order approximation of (6.11) in a weak coupling expansion. Here will see that the same is true for the general class of A-D-E quiver theories we have geometrically engineered in this section.

\section{Renormalization of gauge couplings}

The superpotential (6.11) contains the periods of $\Omega$ over non-compact cycles. These periods are divergent and need a cut off $\Lambda_{0}$ to be well defined. These are long distance (IR) divergences and therefore we expect them to be related to short distance (UV) divergences in the field theory. This was the case for $X\left(k, A_{1}\right)$ [13], where the renormalization of the gauge coupling constant in field theory was forced upon us in the geometric set up by the IR divergence. This is also true for the general A-D-E cases as we now proceed to show.

The periods of $\Omega$ can be computed using the fact that the Calabi-Yau under consideration is an ALE fibration over a complex plane. The three cycles in this geometry project to lines in the $t$-plane where, over each point, there is an $S^{2}$. Compact $S^{3}$ cycles are those for which the projection is a line segment and the holomorphic volume of the $S^{2}$ vanishes at each end. Non-compact cycles on the other hand are semi- infinite lines in the limit when $\Lambda_{0}$ is infinite. The periods can be computed as integrals of the holomorphic volume of a given $S^{2}$ over the path in the $t$-plane, i.e.,

$$
\int_{B_{(p, K)}}^{\Lambda_{0}} \Omega=\int_{C_{(p, K)}} \widetilde{\alpha}\left(\vec{\rho}_{K}\right) d t
$$


where $C_{i}$ is an appropriate contour and $\widetilde{\alpha}\left(\vec{\rho}_{K}\right)$ is the volume (6.5) after the deformations are introduced, so we should have $\widetilde{\alpha}\left(\vec{\rho}_{K}\right) \rightarrow \alpha\left(\vec{\rho}_{K}\right)$ when the deformations are turned off.

Let us expand $\widetilde{\alpha}$ in a Laurent expansion in $t$,

$$
\widetilde{\alpha}\left(\vec{\rho}_{K}\right)=\sum_{m=-\infty}^{\infty} \sigma_{m} t^{m}
$$

The charge of the LHS can be seen to be $k Q(t)$ by setting all deformations to zero. This implies that

$$
Q\left(\sigma_{m}\right)=(k-m) Q(t)=\frac{k-m}{k C_{2}(G)} .
$$

Our aim is to find the possible dependence of $\sigma_{m}$ on the deformation parameters. Recall that deformation parameters $d_{\beta \delta \gamma}$ are the coefficients of the allowed monomials $t^{\beta} y^{\delta} z^{\gamma}$. In the following we will suppress the subscripts since only the charge will be important. The charge of deformation parameters is therefore,

$$
Q(d)=1-Q(\text { monomial }) \geq 1-\frac{\hat{c}}{2}=\frac{k+1}{k C_{2}(G)}
$$

where equality holds for the log normalizable deformations.

Finally, imposing the condition that $\widetilde{\alpha}_{i}(t) \rightarrow \alpha_{i}(t)$ upon turning off the deformations, $d \rightarrow 0$, implies that

$$
\begin{gathered}
\sigma_{m}=0 \quad \text { for } m>k \\
\sum_{m=0}^{k} \sigma_{m}=\alpha\left(\vec{\rho}_{K}\right) \\
\sigma_{-1}=\sum_{i=1}^{r} g_{i} d_{i}^{\text {log }} \quad \text { where } d_{i}^{\text {log }} \text { are log normalizable, }
\end{gathered}
$$

$g_{i}$ are classical superpotential parameters and $\sigma_{m}$ for $m \leq-2$ depend on normalizable as well as log normalizable deformations.

The conclusion is then that the $\Lambda_{0}$ dependence of the non-compact periods is

$$
\Pi_{(p, K)}=\int^{\Lambda_{0}} \widetilde{\alpha}\left(\vec{\rho}_{K}\right) d t=\sum_{i=1}^{r} n_{K}^{i} W_{i}\left(\Lambda_{0}\right)+\sigma_{-1} \log \left(\Lambda_{0}\right)+\mathcal{O}\left(\frac{1}{\Lambda_{0}}\right)+\ldots
$$

The first term on the RHS is an irrelevant constant, which is independent of the deformation parameters. So the only dangerous divergence is the log one, with coefficient $\sigma_{-1}$. The only parameters in the superpotential (6.11) which can be renormalized to absorb 
these $\log$ divergences are the $\alpha_{K}$ 's. It is non-trivial for this to be possible, as the $\alpha_{K}$ 's are the coefficients of very special functions of $S_{(p, K)}$ 's; so we need to show that the $\sigma_{-1}$ 's conspire to give this same $S_{(p, K)}$ dependence.

Let us choose the orientation of all the contours for computing the compact periods to be counter-clockwise and for the non-compact dual periods to go from $\Lambda_{0}$ on the lower sheet to $\Lambda_{0}$ on the upper sheet crossing the branch cuts defined to be between the two points that split when the deformation is tuned on. The notion of upper sheet and lower sheet refers to the fact that for each $S^{3}$ we have a double point on the $t$ plane and the fibered geometry has naturally related to a double covering.

Now we only have to remember that at each point on the $t$-plane we have a fiber with a basis of two cycles intersecting according to the Cartan matrix of the corresponding A-D-E root system. Let us pick one of the non-compact periods $\Pi_{(p, K)}$ and keep track of how it changes as we change $\Lambda_{0} \rightarrow e^{i \theta} \Lambda_{0}$ with $\theta \in\{0,2 \pi\}$.

Using the Picard-Lefschetz formula [40], the cycle corresponding to the positive root $\vec{\rho}_{K}$ will change as the contour crosses the vanishing cycles 3 according to their intersection. This can be made very precise by denoting $\vec{\rho}_{L}$ the class of the compact cycle $\vec{\rho}_{K}$ is crossing. The change in the period is then,

$$
\Delta \Pi_{(p, K)}=\left(\vec{\rho}_{K} \cdot \vec{\rho}_{L}\right) S_{(m, L)}
$$

where $m=1, \ldots, k$ refers to the particular solution of $\sum_{i=1}^{r} n_{L}^{i} W_{i}^{\prime}(t)=0$ which corresponds to the cycle which we are crossing. Now we can write the total change in the non-compact period as $\Lambda_{0}$ goes around as

$$
\Delta \Pi_{(p, K)}=\sum_{L \in \Delta_{+}}\left(\vec{\rho}_{K} \cdot \vec{\rho}_{L}\right) \sum_{m=1}^{k} S_{(m, L)}
$$

This implies that $\Pi_{(p, K)}$ has a logarithmic dependence on $\Lambda_{0}$ as expected:

$$
\Pi_{(p, K)}=\frac{1}{2 \pi i}\left(\sum_{L \in \Delta_{+}}\left(\vec{\rho}_{K} \cdot \vec{\rho}_{L}\right) \sum_{m=1}^{k} S_{(m, L)}\right) \log \left(\Lambda_{0}\right)+\ldots
$$

where ... are the cut-off single valued pieces.

3 Vanishing cycles in Picard-Lefschetz formula refer to cycles that can shrink by changing the complex structure. In our case by setting to zero the deformations. 
Recall that the second term in (6.11) was obtained by the identification $S_{(p, K)} \leftrightarrow$ $\operatorname{Tr}\left(W_{\alpha}^{2}\right)_{(p, K)}$, the $S U\left(M_{(p, K)}\right)$ glueball field. Therefore, $\alpha_{K}$ is also identified with the bare coupling of the corresponding gauge factor $\frac{8 \pi^{2}}{\left(g_{0}^{\mathrm{YM(K)}}\right)^{2}}$. This implies that only $r$ of all $\alpha_{K}$ 's are linearly independent. Let us choose as basis $\alpha_{i}$ with $i \in \Delta^{0}$, the set of simple roots. The other $\alpha_{K}$ 's corresponding to positive roots $\vec{\rho}_{K}=\sum_{i=1}^{r} n_{K}^{i} \vec{e}_{i}$ are given by,

$$
\alpha_{K}=\sum_{i=1}^{r} n_{K}^{i} \alpha_{i}
$$

Clearly, each $\alpha_{i}$ has to have a logarithmic dependence on $\Lambda_{0}$. In order to have a dimensionally sensible expression we need to include new parameters $\Lambda_{i}$, which will be identified with the dynamically generated scales of the high energy $\prod U\left(N_{i}\right)$ theory. Let us assume the simplest ansatz for the basis,

$$
\alpha_{i}=-\frac{8 \pi^{2}}{\left(g_{0}^{\mathrm{YM}(\mathrm{i})}\right)^{2}}=\beta_{i} \log \left(\frac{\Lambda_{i}}{\Lambda_{0}}\right) \quad \text { with } \quad i=1, \ldots, r
$$

where $\beta_{i}$ are yet to be determined. This is the same phenomenon as dimensional transmutation in field theoretic language.

Let us collect the possibly log-divergent pieces of the superpotential (6.11), using the result from (6.12):

$$
-W_{\text {divg }}=\sum_{L \in \Delta_{+}} \sum_{m=1}^{k} S_{(m, L)}\left(\sum_{p=1}^{k} \sum_{K \in \Delta_{+}} M_{(p, K)}\left(\vec{\rho}_{K} \cdot \vec{\rho}_{L}\right) \log \left(\Lambda_{0}\right)+\alpha_{L}\right) .
$$

The $\alpha_{L}$ appearing in the above must cancel these divergences term by term in $L$, requiring that

$$
\alpha_{L}=-\sum_{p=1}^{k} \sum_{K \in \Delta_{+}} M_{(p, K)}\left(\vec{\rho}_{K} \cdot \vec{\rho}_{L}\right) \log \left(\Lambda_{0}\right)+\ldots
$$

where ... denote cut-off independent pieces. Specializing to $L=i \in \Delta^{0}$ and using (6.14) we get that

$$
\beta_{i}=\sum_{K \in \Delta_{+}}\left(\sum_{p=1}^{k} M_{(p, K)}\right)\left(\vec{\rho}_{K} \cdot \vec{e}_{i}\right)=\sum_{j=1}^{r} C_{i j} \sum_{K \in \Delta_{+}}\left(\sum_{p=1}^{k} M_{(p, K)}\right) n_{K}^{i}=\sum_{j=1}^{r} C_{i j} N_{j} .
$$

The geometry has thus reproduced the 1-loop holomorphic beta functions (4.5). 
It is simple to see that with (6.15) and (6.13) the superpotential does not have logarithmic divergences. As a by-product we have learned that the superpotential also depends on $r$ scales $\Lambda_{i}$ in the following form,

$$
W=-\sum_{L \in \Delta_{+}} \hat{\alpha}_{L}\left(\sum_{p=1}^{k} S_{(p, L)}\right)+\ldots
$$

where

$$
\hat{\alpha}_{L}=\sum_{i=1}^{r} n_{L}^{i} \beta_{i} \log \left(\Lambda_{i}\right) \quad \text { with } \quad \vec{\rho}_{L}=\sum_{i=1}^{r} n_{L}^{i} \vec{e}_{i}
$$

\section{Leading order superpotential}

The exact effective superpotential 66.11) can be studied in the weak coupling limit. This means that the dynamically generated scales $\Lambda_{i}$ with $i=1, \ldots, r$ are small compared to the scales set by the superpotentials $W_{i}(t)$ 's. In geometrical terms this means that the compact $S^{3}$ 's are small compared to their separation in the $t$-plane. In order to be more precise let us introduce some notation. For zero deformation parameters we get $k R_{+}$ singular points located at the solutions of

$$
W_{K}^{\prime}(t) \equiv \sum_{i=1}^{r} n_{K}^{i} W_{i}^{\prime}(t) \equiv g_{K} \prod_{p=1}^{k}\left(t-a_{(p, K)}\right)=0
$$

for $K \in \Delta^{+}$, the set of positive roots.

After the deformation each singular point $t=a_{(p, K)}$ splits into two giving rise to $S_{(p, K)}^{3}$. Let us denote the new two points by $a_{(p, K)}^{+}$and $a_{(p, K)}^{-}$. Now the periods can be written more explicitly as follows,

$$
S_{(p, K)}=\frac{1}{2 \pi i} \int_{a_{(p, K)}^{-}}^{a_{(p, K)}^{+}} \widetilde{\alpha}\left(\vec{\rho}_{K}\right) d t \quad \text { and } \quad \Pi_{(p, K)}=\frac{1}{2 \pi i} \int_{a_{(p, K)}^{+}}^{\Lambda_{0}} \widetilde{\alpha}\left(\vec{\rho}_{K}\right) d t
$$

The weak coupling regime can therefore be defined by the following conditions $\left|a_{(p, K)}^{+}-a_{(p, K)}^{-}\right| \ll\left|a_{(m, L)}-a_{(p, K)}\right|$ for all $(p, K) \neq(m, L)$.

Following [13], using monodromy arguments one can compute the $\log \left(S_{(p, K)}\right)$ and $\log \left(a_{(p, K)}-a_{(m, L)}\right)$ dependence of $\Pi_{(p, K)}$ and therefore of the superpotential (6.11). 
Consider first the geometry close to $a_{(p, K)}$, this geometry can be thought of as that of a single conifold in the limit we are considering, therefore, the $S_{(p, K)}$ period should look like [13],

$$
S_{(p, K)}=\frac{1}{2 \pi i} W_{K}^{\prime \prime}\left(a_{(p, K)}\right) \int_{a_{(p, K)}^{-}}^{a_{(p, K)}^{+}} \sqrt{\left(t-a_{(p, K)}\right)^{2}-\mu_{\mathrm{eff}}} d t
$$

Using Picard-Lefschetz formula for $\mu_{\text {eff }} \rightarrow e^{2 \pi i} \mu_{\text {eff }}$, we get that the corresponding dual period changes as $\Delta \Pi_{(p, K)}=S_{(p, K)}$, therefore one can conclude that,

$$
\Pi_{p, K}=\frac{1}{2 \pi i} S_{(p, K)} \log \frac{S_{(p, K)}}{W_{K}^{\prime \prime}\left(a_{(p, K)}\right)}+\ldots
$$

Finally, let us consider how $\Pi_{(p, K)}$ changes when we move one $a_{(q, L)}$ around $a_{(p, K)}$, again using P-L formula gives that,

$$
\Delta \Pi_{(p, K)}=\left(\overrightarrow{\rho_{K}} \cdot \overrightarrow{\rho_{L}}\right) S_{(q, L)}
$$

Notice that the coefficient in front of $S_{q, L}$ does not depend on $m$ or $p$, this is because the intersection formula only sees the classes and for a given $K$ all $p$ have the same class.

Now we can collect all these partial results to write,

$2 \pi i \Pi_{(p, K)}=S_{(p, K)} \log \ln \frac{S_{(p, K)}}{W_{K}^{\prime \prime}\left(a_{(p, K)}\right)}+\sum_{L \in \Delta^{+}} \sum_{m=1}^{k}\left(\overrightarrow{\rho_{K}} \cdot \overrightarrow{\rho_{L}}\right) S_{(q, L)} \log \left(a_{(p, K)}-a_{(q, L)}\right)+\ldots$

in this formula the sum over $L$ and $m$ runs over all $(q, L) \neq(p, K)$.

The leading order superpotential can then be obtained by combining (6.11), (6.17), and $(6.16)$ to get,

$$
\begin{aligned}
W= & \sum_{K} \sum_{p=1}^{k} M_{(p, K)}\left(S_{(p, K)} \log \frac{W_{K}^{\prime \prime}\left(a_{(p, K)}\right)}{S_{(p, K)}}+\sum_{L} \sum_{m=1}^{k}\left(\sum_{i, j=1}^{r} C_{i j} n_{K}^{i} n_{L}^{j}\right) S_{(q, L)} \log \frac{1}{\left(a_{(p, K)}-a_{(q, L)}\right)}\right) \\
& +\sum_{K}\left(\sum_{p=1}^{k} M_{(p, K)}\right) \sum_{i, j=1}^{r}\left(C_{i j} n_{K}^{j} \log \Lambda_{i}\right) \sum_{L} n_{L}^{i} \sum_{m=1}^{k} S_{(q, L)}+\ldots
\end{aligned}
$$

In order to compare with the gauge theory answer from naive integrating in, let us write $W$ collecting all terms with $S_{K}$ together,

$$
\begin{aligned}
W & =\sum_{(p, K)} S_{(p, K)}\left(M_{(p, K)} \ln \left(\frac{W_{K}^{\prime \prime}\left(a_{(p, K)}\right) \prod_{i} \Lambda_{i}^{n_{K}^{i} n_{K}^{j} C_{i j}}}{S_{(q, K)}}\right)\right) \\
& +\sum_{(p, K)} \sum_{(q, L) \neq(p, K)} \sum_{i, j=1}^{r} M_{(p, K)} S_{(q, L)} n_{L}^{i} n_{K}^{j} C_{i j} \ln \left(\frac{\Lambda_{i}}{a_{(p, K)}-a_{(q, L)}}\right)
\end{aligned}
$$


We thus find perfect agreement with the gauge theory answer (5.13). Notice that (5.13) contains linear terms in $S_{(p, K)}$. These and possibly an infinite power expansion in $S_{(p, K)}$ 's can not be derived using monodromy arguments. A more detailed analysis of the geometry result shows that the superpotential indeed generally contains an infinite power expansion of terms which are missed by the naive integrating in analysis, as was computed for $X\left(k, A_{1}\right)$ in 13$]$.

Finally, one has to check that the weak coupling approximation is self-consistent. For this it is necessary to identify the expansion parameters that enter in the infinite power series mentioned before. Let us assume that all the relevant scales set by the classical superpotentials are of the same order equal to $\Delta$. This means that $\left(a_{(p, K)}-a_{(q, L)}\right) \sim$ $W_{J}^{\prime \prime} \sim \Delta$ for all $K, L, J \in \Delta_{+}$. Moreover, let us assume that all the scales of the individual $U\left(N_{i}\right)$ factors are of the same order $\Lambda_{i} \sim \Lambda \ll \Delta$ for $i=1, \ldots, r$. Let us show that the natural dimensionless expansion parameter for the computation of periods is $\frac{\Lambda}{\Delta}$.

The leading order superpotential (5.1) implies that $\left\langle S_{(p, K)}\right\rangle^{M_{(p, K)}}=\Lambda_{(p, K)}^{3 M_{(p, K)}}$. Then, using (5.11) with $W_{K}^{\prime \prime} \sim\left(a_{(p, K)}-a_{(q, L)}\right) \sim \Delta$, and taking all $\Lambda_{i} \sim \Lambda$, we find for the expectation value of the gaugino fields, or in geometric language, the sizes of the $S^{3}$ cycles:

$$
\left(\frac{\left\langle S_{K}\right\rangle}{\Delta^{3}}\right)^{M_{K}}=\left(\frac{\Lambda}{\Delta}\right)^{\sum_{J} M_{J} \vec{\rho}_{J} \cdot \vec{\rho}_{K}}
$$

This implies that the power expansion in $\Lambda / \Delta$, and hence the superpotential (5.13), are valid approximations when $\sum_{J} M_{J} \vec{\rho}_{J} \cdot \vec{\rho}_{K}>0$. Since

$$
\sum_{J} M_{J} \vec{\rho}_{J} \cdot \vec{\rho}_{K}=\sum_{j=1}^{r} n_{K}^{j}\left(\sum_{i=1}^{r} N_{i} C_{i j}\right)=\sum_{j=1}^{r} n_{K}^{i} \beta_{i}
$$

with $n_{K}^{i} \geq 0$, and $n_{j}^{i}=\delta_{j}^{i}$ for $K=j$ a simple root, the necessary condition is thus that all $U\left(N_{i}\right)$ 's have to be asymptotically free.

This analysis shows that in cases when no weak coupling expansion is possible in terms of the parameters of a given theory two possibilities can occur. The first is that the exact superpotential (6.11) might still be computable in a power expansion in terms of the parameters of a different (dual) theory and the second is that no simple gauge theoretic interpretation exists even though the geometric description still yields exact results. 


\section{Duality Predictions From Geometric Construction of the A-D-E Quiver Theories}

Consider the geometric engineering of the quiver theory. Consider blowing down the cycles (i.e. where the inverse couplings $1 / g_{i}^{2}=0$ ). If we are just given this geometry together with some data about which classes the branes wrap (or how much flux is coming out of each vanishing $S^{3}$ ) we cannot uniquely determine the quiver theory corresponding to it. The reason for this is that in order to decipher the gauge theory we have to identify certain parameters in the geometry with a choice of simple roots of the A-D-E, and this is unique only up to the choice of a Weyl group action. This implies that with this data we cannot quite give a unique description of the quiver theory, however we can give descriptions in seemingly different looking gauge theories which have to be equivalent because they are describing the same underlying string theory. Our constructions apply equally well to A$\mathrm{D}-\mathrm{E}$ as well as the affine case. This is how geometry predicts gauge theory dualities, in one to one correspondence with elements of the Weyl group. As is well known the Weyl group is generated by Weyl reflections about simple roots, and this we identify as Seiberg-like dualities in the corresponding quiver theory.

In the original geometric engineering we have blown up $S^{2}$ 's and which $S^{2}$ 's we blowup picks a particular 'preferred' description for which gauge couplings $1 / g_{i}^{2}>0$. Of course they can be viewed as analytic continuation of the other dual descriptions where some of the gauge couplings squared are negative. This phenomenon, taking into account the dimensional transmutation, becomes part of the data of matching of scales between the dual theories.

Let us consider a given theory with branes $N_{i}$ wrapping the corresponding dual cycles, undergoing a transition to Higgs branch with branch number degeneracies $M_{(p, K)}$ where $K$ labels the positive roots and $p$ an integer between $1, \ldots, k$. Now consider a different choice of positive roots given by Weyl reflection about $\vec{e}_{i_{0}}$. This affects the roots by

$$
\vec{e}_{j}^{\prime}=\vec{e}_{j}-\left(\vec{e}_{j} \cdot \vec{e}_{i_{0}}\right) \vec{e}_{i_{0}} .
$$

The conservation of brane charge determines the rank of the gauge groups after transitions, as in [3,4] and we find

$$
\sum N_{i} \vec{e}_{i}=\sum N_{i}^{\prime} \vec{e}_{i}^{\prime} .
$$

It follows from this that $N_{j}^{\prime}=N_{j}$ for $j \neq i_{0}$, and

$$
N_{i_{0}}^{\prime}=N_{f}-N_{i_{0}}
$$


where $N_{f}=\sum_{i \neq i_{0}}\left(-\vec{e}_{i} \cdot \vec{e}_{i_{0}}\right) N_{i}$ denotes the number of flavors of the $U\left(N_{i_{0}}\right)$ theory. The Weyl group also acts on the couplings, which correspond to Kahler volumes of the $e_{i}^{\prime}$, as

$$
\frac{1}{{g^{\prime}}^{2}}=\frac{1}{g_{i}^{2}}-\frac{\vec{e}_{i} \cdot \vec{e}_{i_{0}}}{g_{i_{0}}^{2}} .
$$

Similarly it acts on the superpotentials by the integral of the holomorphic 3 -form over the relevant cycle which is

$$
W_{i} \rightarrow W_{i}-\left(\vec{e}_{i} \cdot \vec{e}_{i_{0}}\right) W_{i_{0}}
$$

In the IR, i.e. at scales below the scale of the superpotential we also have to choose which branches we are in. This makes sense assuming that the coupling of the gauge theory is weak at the scale of the superpotential, so that the classical analysis is reliable. In this case we have branches labeled by the positive roots $\vec{\rho}_{K}$. Under the Weyl reflection the positive roots get permuted except for $\vec{\rho}_{K}=\vec{e}_{i_{0}}$ which goes to minus itself (it is also easy to see, using (7.4) that the choices within a given branch get mapped in a canonical way). Thus $M_{p, K}=M_{p, w_{e_{i}}}^{\prime}(K)$, for $K \neq e_{i_{0}}$ where $w_{e_{i_{0}}}$ denotes the Weyl reflection by $e_{i_{0}}$, and $M_{p, K}^{\prime}=-M_{p, K}$ for $K=e_{i_{0}}$. Note that this latter action on the branches would yield negative multiplicities unless $M_{p, e_{i}}=0$. So only for this case we can formally use the dual. We will elaborate on the geometric meaning of this later. However, we emphasize that even if $M_{p, e_{i}} \neq 0$ in a formal sense the dual theory makes sense. What we mean by this is that when we set up the dual geometry and write the corresponding superpotential, replacing the flux coming from the branch corresponding to $\vec{e}_{i_{0}}$ with a negative number does make sense, and would yield an identical description of the geometry. Thus at the level of setting up the dual geometry description we simply have an ambiguity of reading off the gauge theory. Thus the geometry predicts gauge theoretic dualities which we will verify in the next section.

\section{Dualizing a gauge group factor}

Consider a particular $U\left(N_{i_{0}}\right)$ gauge group factor in our general $\mathcal{N}=1$ quiver labelled by $k$ and $G$ or $\widehat{G}$. We write the superpotential for the fields charged under $U\left(N_{i_{0}}\right)$ as

$$
W=\frac{s}{k+1} \operatorname{Tr} \phi^{k+1}+\operatorname{Tr} \phi \bar{Q} Q+\operatorname{Tr} m Q \bar{Q},
$$

where $Q$ is a $N_{f} \times N_{c}$ matrix, with $\bar{Q} Q$ in the adjoint of $U\left(N_{c}\right)$ singlet under $U\left(N_{f}\right)$ and $M=Q \bar{Q}$ a $U\left(N_{c}\right)$ singlet and in the adjoint of $U\left(N_{f}\right)$. The $N_{f}=\sum_{i \neq i_{0}}\left(-\vec{e}_{i} \cdot \vec{e}_{i_{0}}\right) N_{i}$ 
fundamentals arise from the bi-fundamentals connecting to the neighboring nodes of the quiver diagram, and the mass $m$ in (8.1) is a matrix in the flavor space, which is actually given by the expectation values of the adjoints of the neighboring nodes' gauge groups. We treat the neighboring nodes as weakly gauged flavor symmetries.

As we briefly review, the above theory can be dualized to a $U\left(N_{f}-N_{i_{0}}\right)$ gauge theory for all $k$. This is naturally related to the $U\left(N_{f}-N_{i_{0}}\right)$ which arises in the $\mathcal{N}=2$ theory (setting $s=0$ in (8.1)) at the base where the "baryon branch" intersects the Coulomb branch [16]. Before discussing the details of the duality, we note a few of the most important features.

As seen in the geometry, the duality corresponds to a $G$ Weyl reflection, or $\widehat{G}$ Weyl reflection in the affine case. The duality does not act on the $N_{i}$ of the other nodes, which correspond to unchanged flavor symmetries, and takes $N_{i_{0}} \equiv N_{c}$ to $N_{f}-N_{c}$, i.e.

$$
N_{i}{ }^{\prime}=N_{i} \quad \text { for } \quad i \neq i_{0}, \quad N_{i_{0}}{ }^{\prime}=N_{i_{0}}-\sum_{j} \vec{e}_{i_{0}} \cdot \vec{e}_{j} N_{j}
$$

As discussed in the previous section, we can write this as

$$
\vec{N} \equiv \sum_{i=1}^{r} N_{i} \vec{e}_{i}=\sum_{i=1}^{r} N_{i}^{\prime} \vec{e}_{i}^{\prime}
$$

with

$$
\vec{e}_{i}^{\prime}=\vec{e}_{i}-\left(\vec{e}_{i_{0}} \cdot \vec{e}_{i}\right) \vec{e}_{i_{0}}
$$

which is precisely the action of a Weyl reflection about the simple root $\vec{e}_{i_{0}}$. Such transformations for all the nodes generate the entire Weyl group (or affine Weyl group for the case of the affine quiver diagrams).

To see how (7.3) occurs in the field theory duality, consider the holomorphic beta functions of the $\mathcal{N}=1$ quiver diagram theories (above the scale $\Delta$ where the adjoints get masses); these coincide with (4.1), and can be written as in (4.2). The beta functions of the theory after the duality transformation are

$$
\beta_{i}{ }^{\prime}=\vec{e}_{i} \cdot \sum_{j} \vec{e}_{j} N_{j}{ }^{\prime}=\vec{e}_{i}{ }^{\prime} \cdot \sum_{j} \vec{e}_{j}{ }^{\prime} N_{j}{ }^{\prime}=\vec{e}_{i}{ }^{\prime} \sum_{j} \vec{e}_{j} N_{j}=\beta_{i}-\left(\vec{e}_{i_{0}} \cdot \vec{e}_{i}\right) \beta_{i_{0}},
$$

where we used (8.4), $\vec{e}_{i}{ }^{\prime} \cdot \vec{e}_{j}{ }^{\prime}=\vec{e}_{i} \cdot \vec{e}_{j}=C_{i j}$, and (8.3). So the holomorphic functions transform precisely as under the Weyl transformation (8.4). We can formally integrate these beta functions to get the similar transformation of the couplings $g_{i}^{(-2)}$, as in (7.3). 
A similar transformation as (8.5) would hold for the exact physical beta functions (4.7) if all $\gamma\left(\phi_{i}\right)$ are equal and $\beta\left(\lambda_{i j}\right)=0$; likewise for (4.9) if $\widehat{\gamma}\left(Q_{i j}\right)=0$.

Consider matching the running gauge couplings, given by (4.5), before and after the duality transformation on some particular $U\left(N_{i_{0}}\right)$; the matching occurs at the scale $\mu=\Lambda_{i_{0}}$ where $U\left(N_{i_{0}}\right)$ gets strong:

$$
e^{2 \pi i \tau_{j}(\mu)}=\left(\frac{\Lambda_{j}}{\mu}\right)^{\beta_{j}}=\left(\frac{\Lambda_{j}^{\prime}}{\mu}\right)^{\beta_{j}^{\prime}} \text { at } \mu=\Lambda_{i_{0}} .
$$

Using (8.5) the matching relation obtained from (8.6) is

$$
\Lambda_{i}^{\prime \beta_{i}^{\prime}}=\Lambda_{i}^{\beta_{i}} \Lambda_{i_{0}}^{-\left(\vec{e}_{i} \cdot \vec{e}_{i_{0}}\right) \beta_{i_{0}}},
$$

i.e. (aside from the case $\widehat{A}_{1}$ where $C_{01}=-2$ )

$$
\Lambda_{i_{0}}^{\beta_{i_{0}}} \Lambda_{i_{0}}^{\prime} \beta_{i_{0}}^{\prime}=1, \quad \Lambda_{j}^{\prime \beta_{j}^{\prime}}=\Lambda_{i_{0}}^{\beta_{i_{0}}\left|s_{i_{0} j}\right|} \Lambda_{j}^{\beta_{j}} \quad j \neq i_{0}
$$

The first relation (8.8), which gives $\Lambda_{i_{0}}=\Lambda_{i_{0}^{\prime}}$, is similar to the duality relation [1] for $\mathcal{N}=1$ SQCD without the adjoint $\phi$

$$
\Lambda_{S Q C D}^{3 N_{c}-N_{f}} \widetilde{\Lambda}_{S Q C D}^{3 \widetilde{N}_{c}-N_{f}} \sim \mu^{N_{f}}
$$

where $\mu$ is the scale appearing in the dual superpotential as $W_{\text {mag }}=\mu^{-1} M q \bar{q}$. Indeed, for $k=1$ the adjoint $\phi_{i_{0}}$ has mass $m=s$ from (8.1) and can be integrated out from both the electric and magnetic theories, giving $\Lambda_{S Q C D}^{3 N_{c}-N_{f}}=m^{N_{c}} \Lambda_{i_{0}}^{\beta_{i_{0}}}$ and $\widetilde{\Lambda}_{S Q C D}^{3 \widetilde{N}_{c}-N_{f}}=m^{\widetilde{N}_{c}} \Lambda_{i_{0}}^{\prime} \beta_{i_{0}}^{\prime}$, and then (8.8) agrees with (8.9) for $\mu=m$.

Integrating the beta function equations, in order to have all $g_{i}^{-2} \geq 0$, we should have

$$
\Lambda_{N A F}>\mu>\Lambda_{A F}
$$

where $\mu$ is the energy scale and $\Lambda_{N A F}$ is the dynamical scale $\Lambda_{i}$ of those $i$ which are not asymptotically free, $\beta_{i}<0$, and $\Lambda_{A F}$ is that of those $i$ which are. In particular, the $\Lambda_{N A F}>\Lambda_{A F}$. As we lower the scale $\mu$, eventually we get to $\Lambda_{i_{0}}$ of the asymptotically free $U\left(N_{i_{0}}\right)$, which we dualize as above. According to (8.5), $U\left(N_{i_{0}}^{\prime}\right)$ is not asymptotically free and $U\left(N_{i}\right)$ is more asymptotically free than it was before if nodes $i$ and $i_{0}$ are linked. The relation (8.7) ensures that the new scales satisfy (8.10), e.g. if $U\left(N_{j}\right)$ is NAF we have $\Lambda_{j}>\Lambda_{i_{0}}$ and then we get $\Lambda_{j}^{\prime}>\Lambda_{i_{0}}$ if $U\left(N_{j}^{\prime}\right)$ is NAF or $\Lambda_{j}^{\prime}<\Lambda_{i_{0}}$ if $U\left(N_{j}^{\prime}\right)$ is AF. 
A final relation, which will occupy the rest of this section is the transformation (7.4) of the superpotential:

$$
W_{i}\left(\phi_{i}\right) \rightarrow W_{i}\left(\phi_{i}\right)-\left(\vec{e}_{i} \cdot \vec{e}_{i_{0}}\right) W_{i_{0}}\left(\phi_{i}\right)
$$

To show that this is indeed the case, we need to show that the dual of our theory (8.1) for the $U\left(N_{i_{0}}\right)$ charged fields is $U\left(N_{f}-N_{i_{0}}\right)$ with the superpotential

$$
\widetilde{W}=-\frac{s}{k+1} \operatorname{Tr} \widetilde{\phi}^{k+1}+\frac{s}{k+1} \operatorname{Tr} m^{k+1}+\operatorname{Tr} \widetilde{\phi} q \bar{q}+\operatorname{Tr} m \bar{q} q
$$

Here $\widetilde{\phi}$ is the $U\left(N_{f}-N_{i_{0}}\right)$ adjoint of the dual theory and $q$ and $\bar{q}$ are the $N_{f}$ dual matter fields. The opposite sign of the first term in (8.12), as compared with (8.1), corresponds to the result of (8.11) for $i=i_{0}: W_{i_{0}} \rightarrow-W_{i_{0}}$. The transformation in (8.11) for the nodes $i \neq i_{0}$ corresponds to the second term in (8.12). This is because the mass $m$ in (8.1) are actually the adjoints $\phi_{i}$ of the nodes linked to $i_{0}$, so the second term in (8.12) will properly lead to (8.11) for the nodes $i \neq i_{0}$ with $\vec{e}_{i} \cdot \vec{e}_{i_{0}}=-1$.

We will first outline how the predicted superpotential (8.12) indeed arises for the case of $k=1$; after that we'll discuss $k>1$.

\section{1. $k=1$ case}

Consider first the case $k=1$, where $\phi_{i}$ is massive, with mass $s$, and can be integrated out for scales $\mu<s$. The relevant duality for the low-energy theory is then that of [24]. When $s$ is large, the low energy theory is $\mathcal{N}=1 \mathrm{SQCD}$ with $N_{f}$ flavors and the additional tree-level superpotential

$$
W_{\text {elec }}=-\frac{1}{2 s} \operatorname{Tr}(\bar{Q} Q)^{2}+\operatorname{Tr} m Q \bar{Q},
$$

obtained by integrating out $\phi$ from (8.1) via its equation of motion. For $s$ large it's a good description to simply add this extra superpotential to the usual SQCD dynamics.

For $N_{f}>N_{c}$, we can dualize the SQCD theory [24] to $U\left(N_{f}-N_{c}\right)$, with superpotential

$$
W_{m a g}=\frac{1}{\mu} M \bar{q} q-\frac{1}{2 s} \operatorname{Tr} M^{2}+\operatorname{Tr} m M
$$

$M$ is massive and can be integrated out by its equation of motion, $M=s\left(\mu^{-1} \bar{q} q+m\right)$, leading to

$$
W_{\text {mag }}=\frac{s}{2} \operatorname{Tr}\left(m+\frac{1}{\mu} \bar{q} q\right)^{2}=\frac{s}{2 \mu^{2}} \operatorname{Tr}(\bar{q} q)^{2}+\frac{s}{\mu} \operatorname{Tr}(m \bar{q} q)+\frac{1}{2} s \operatorname{Tr} m^{2} .
$$


Taking $\mu=s$, this superpotential is precisely what we would obtain from (8.12) upon integrating out the massive adjoint $\widetilde{\phi}$. In particular, corresponding to the Weyl reflection, the sign of the quartic term in (8.15) is opposite to that of 8.13 , and we have the additional term $W_{i}(m)$ in 8.15$)$.

As an aside, we briefly review the vacuum structure of the $U\left(N_{c}\right)$ theory with superpotential (8.1), for $k=1$, thinking of the linked nodes as a $U\left(N_{f}\right)$ flavor symmetry. The relevant detailed analysis has been presented in [42,43]. A semi-classical analysis of the vacua, for general quark masses $m$ leads to $\left(\begin{array}{c}N_{f} \\ r\end{array}\right)$ vacua where the gauge group is Higgsed as $U\left(N_{c}\right) \rightarrow U\left(N_{c}-r\right)$ for $r=0, \ldots, \min \left(N_{c}, N_{f}\right)$; each unbroken $S U\left(N_{c}-r\right)$ has no massless flavors and thus has $N_{c}-r$ susy vacua via gaugino condensation.

Consider the quantum theory in the limit of large $s$, where we simply add (8.13) to the usual $U\left(N_{i_{0}}\right)$ dynamics. For example, for $N_{f}<N_{c}$ the theory is described by the mesons $M$ with superpotential

$$
W=-\frac{1}{2 s} \operatorname{Tr} M^{2}+\operatorname{Tr} m M+\left(N_{c}-N_{f}\right)\left(\frac{s^{N_{c}} \Lambda^{2 N_{c}-N_{f}}}{\operatorname{det} M}\right)^{1 /\left(N_{c}-N_{f}\right)} .
$$

This superpotential has $\frac{1}{2}\left(2 N_{c}-N_{f}\right)\left(\begin{array}{c}N_{f} \\ r\end{array}\right)$ vacua where $\langle M\rangle$ expectation values break $U\left(N_{f}\right) \rightarrow U\left(N_{f}-r\right) \times U(r)$, even in the $m \rightarrow 0$ limit, for every $r=0, \ldots, N_{f}$. These give all the vacua for $N_{f}<N_{c}$ [42]. For $N_{f}>N_{c}$ we can analyze the vacua using the $U\left(N_{f}-N_{c}\right)$ dual. The result (see [42]) are vacua of two types. One type is visible semi-classically in the dual theory, with $U\left(N_{f}-N_{c}\right)$ Higgsed to $U\left(N_{f}-N_{c}-r\right)$ and $U\left(N_{f}\right)$ is unbroken in the $m \rightarrow 0$ limit. The other comes from strong coupling dynamics in the dual theory: when $\operatorname{rank}(M)=N_{f}$, the dual quarks are all massive and a dynamical superpotential is generated in the dual, e.g. via gaugino condensation; as usual, this superpotential is the continuation of (8.16) to $N_{f}>N_{c}$. These vacua again have $U\left(N_{f}\right) \rightarrow U\left(N_{f}-r\right) \times U(r)$ for $r=0, \ldots, N_{f}$.

One can also analyze the problem in the limit where the adjoint mass $s \ll \Lambda$, with $\Lambda$ the scale of the theory with $\phi$ included. The theory can then be usefully analyzed in terms of the curve of the $\mathcal{N}=2$ SQCD theory, breaking to $\mathcal{N}=1$ by the small adjoint mass $s$. This analysis again shows two sorts of vacua [16,42]. One set, existing for all $N_{f}$, are vacua with the entire gauge group confined, and the flavor symmetry broken as $U\left(N_{f}\right) \rightarrow U\left(N_{f}-r\right) \times U(r)$ for all $r \leq\left[N_{f} / 2\right]$ via monopole condensation. The other set exists for $N_{f}>N_{c}$ and have unbroken $U\left(N_{f}\right)$; they are visible semi-classically in the dual $U\left(N_{f}-N_{c}\right)$ theory of $[16]$. 


\section{2. $k>1$}

Now consider (8.1) with $k>1$. As discussed in [44] for $k=2$ and more generally in [45], these theories, without the term $m \bar{Q} Q$ in (8.1), are dual to a $U\left(N_{f}-N_{i}\right)$ theory with superpotential

$$
\bar{W}=-\frac{s}{k+1} \operatorname{Tr} \widetilde{\phi}_{i}^{k+1}+\operatorname{Tr} \widetilde{\phi} q \bar{q}
$$

(Comparing with [45], we have normalized $\bar{q}$ and $q$ so that the coefficient of the Yukawa term in (8.17) is the same as in the electric theory.) As discussed in [45], this duality can be obtained from that of [46,47,33 by deforming by the $\bar{Q} \phi_{i} Q$ term in (8.1). The dual theory is of the same form as the original theory, and does not contain the gauge singlet mesons found in the original $\mathcal{N}=1$ dualities of [24,46,47,33]; all of the mesons usually required in the dual are massive for $0 \neq s_{i}<\infty$ [45].

Following the $\operatorname{Tr} m Q \bar{Q}$ in (8.1) through the duality is a little more involved. As was discussed in [44] for the case $k=2$, one finds various vacua. Our interest is in showing that one of these vacua has $U\left(N_{f}-N_{c}\right)$ gauge group, with the terms involving $m$ in the superpotential, as in (8.12).

As in [45], we obtain the duality by flowing from that of 46, 47,33, which relates the theory 8.13$)$ to a magnetic $U\left(k N_{f}-N_{c}\right)$ theory with superpotential

$$
\bar{W}=-\frac{s}{k+1} \operatorname{Tr} Y^{k+1}+\frac{s}{\mu^{2}} \sum_{j=1}^{k} M_{j} \widetilde{q} Y^{k-j} q+\lambda M_{2}+m M_{1}
$$

The $\operatorname{Tr} \phi \bar{Q} Q$ perturbation in (8.18), with coefficient $\lambda$ which we'll take to equal $1_{N_{f}}$, leads to a Higgsing of the magnetic theory to $U\left(N_{f}-N_{c}\right)$ [45]. We now consider the effect of the added $m$ perturbation in (8.18). The $F$-term conditions required for a vacuum of the theory 8.18 ) are

$$
\begin{aligned}
\frac{s_{i}}{\mu^{2}} \widetilde{q} Y^{p} q & =-m \delta_{p, k-1}-\lambda \delta_{p, k-2}, \\
Y^{k} & =\mu^{-2} \sum_{j=1}^{k-1}(k-j) M_{j} q \widetilde{q} Y^{k-j-1}, \\
\sum_{j=1}^{k} M_{j} \widetilde{q} Y^{k-j} & =0 .
\end{aligned}
$$

The vacuum solution of [45] for $m=0$ Higgses $U\left(k N_{f}-N_{c}\right)$ to $U\left(N_{f}-N_{c}\right)$. This solution can now be modified to account for $m \neq 0$. For simplicity, we just discuss the case $k=2$. Considering first the first flavor, the vacuum of [45] has $q_{1}^{\alpha}=b \delta^{\alpha, 1}$ and $\widetilde{q}_{\alpha}^{1}=b \delta_{\alpha 1}$, with 
$b^{2}=-\lambda_{1} \mu^{2} / s$, satisfying (8.19) for $p=0$. We can satisfy (8.19) for $p=1$ by taking $Y_{1}^{1}=-m_{1} / \lambda_{1}$. In order to satisfy the other equations in (8.19) we also need $\left(M_{1}\right)_{1}^{1}$ and $\left(M_{2}\right)_{1}^{1}$ to be non-zero; these non-zero values will not contribute to the low-energy superpotential, since the linearity of (8.18) in the $M_{j}$ ensures that the coefficients of the $M_{j}$ have zero expectation value.

We now expand (8.18) around this vacuum, where $U\left(2 N_{f}-N_{c}\right)$ is Higgsed to $U\left(2 N_{f}-\right.$ $\left.N_{c}-1\right)$. Though the $q_{1}$ and $\widetilde{q}^{1}$ flavor is eaten, we get back a flavor from $F^{\alpha} \sim Y_{1}^{\alpha}$ and $\bar{F}_{\alpha} \sim Y_{\alpha}^{1}$. Expanding out the $-\frac{s}{3} \operatorname{Tr} Y^{3}$ term of the $U\left(2 N_{f}-N_{c}\right)$ theory gives

$$
-\frac{s}{3} \operatorname{Tr} Y^{3} \rightarrow-\frac{s}{3}\left(\left(-\frac{m_{1}}{\lambda_{1}}\right)^{3}+\operatorname{Tr} \widehat{Y}^{3}\right)+\lambda_{1} \operatorname{Tr} \bar{F} \widehat{Y} F+m_{1} \bar{F} F
$$

where $\widehat{Y}$ is the part of $Y$ in the unHiggsed $U\left(2 N_{f}-N_{c}-1\right)$ adjoint, and $F$ has been normalized so that the Yukawa coupling in (8.20) ccoefficient is $\lambda_{1}$. Continuing this process for all flavors, and taking the $\lambda=1_{N_{f}}$, we eventually get a $U\left(N_{f}-N_{c}\right)$ theory with superpotential precisely as in (8.12), just as we wanted to verify.

We can also see the above $U\left(N_{f}-N_{c}\right)$ dual in the limit where we treat the coefficient $s$ of the $\mathcal{N}=2 \rightarrow \mathcal{N}=1$ superpotential term in (8.1) as being small, via an analysis similar to that of [16]. In the undeformed $\mathcal{N}=2$ theory, at the root of the baryon branch, there is a free-magnetic $U\left(N_{f}-N_{c}\right) \times U(1)^{2 N_{c}-N_{f}}$ theory. Deforming by the term $W_{i}=\frac{s}{k+1} \operatorname{Tr} \phi^{k+1}$ leads to various vacua, the one of interest for us being that where the $U\left(N_{f}-N_{c}\right)$ remains unbroken and the $U(1)^{2 N_{c}-N_{f}}$ is Higgsed entirely by monopoles, which condense due to the $W_{i}=\frac{s}{k+1} \operatorname{Tr} \phi^{k+1}$ deformation. Carrying out this analysis along the lines of [16], it can be seen how all the terms in the expected superpotential (8.12) can indeed arise.

\section{9. $A_{2}$ example}

In this section we study the $A_{2}$ quiver theory with $k=1$ as an example of how the dualities enter the description of the theory both in the field theory analysis and in the geometric analysis. We first present the field theory analysis and then discuss how it is realized geometrically. The other A-D-E cases work in a similar fashion. 


\subsection{QFT analysis of $A_{2}$ with $k=1$}

Consider the $A_{2}$ quiver theory $U\left(N_{1}\right) \times U\left(N_{2}\right)$ for $k=1$, i.e.

$$
W_{i}\left(\phi_{i}\right)=m_{i}\left(\frac{1}{2} \operatorname{Tr} \phi_{i}^{2}-a_{i} \operatorname{Tr} \phi_{i}\right)
$$

for $i=1,2$. As in (5.8), vacua have

$$
U\left(N_{1}\right) \times U\left(N_{2}\right) \rightarrow U\left(M_{1}\right) \times U\left(M_{2}\right) \times U\left(M_{3}\right),
$$

with $N_{1}=M_{1}+M_{3}$ and $N_{2}=M_{2}+M_{3}$ and $U\left(M_{3}\right)$ is diagonally embedded in $U\left(N_{1}\right) \times$ $U\left(N_{2}\right)$. In these vacua $\phi_{1}=\operatorname{diag}\left(a_{1} 1_{M_{1}}, a_{3} 1_{M_{3}}\right)$ and $\phi_{2}=\operatorname{diag}\left(a_{2} 1_{M_{2}}, a_{3} 1_{M_{3}}\right)$ with $a_{3}=$ $\left(m_{1} a_{1}+m_{2} a_{2}\right) /\left(m_{1}+m_{2}\right)$ (using (5.2) ). Using (5.11) the low energy scales are

$$
\begin{aligned}
\Lambda_{M_{1}}^{3 M_{1}} & =m_{1}^{M_{1}}\left(a_{1}-a_{3}\right)^{-M_{3}}\left(a_{1}-a_{2}\right)^{M_{2}} \Lambda_{N_{1}}^{2 M_{1}-M_{2}+M_{3}}, \\
\Lambda_{M_{2}}^{3 M_{2}} & =m_{2}^{M_{2}}\left(a_{2}-a_{3}\right)^{-M_{3}}\left(a_{2}-a_{1}\right)^{M_{1}} \Lambda_{N_{2}}^{2 M_{2}-M_{1}+M_{3}}, \\
\Lambda_{M_{3}}^{3 M_{3}} & =m_{3}^{M_{3}}\left(a_{3}-a_{1}\right)^{-M_{1}}\left(a_{3}-a_{2}\right)^{-M_{2}} \Lambda_{N_{1}}^{M_{3}-M_{2}+2 M_{1}} \Lambda_{N_{2}}^{M_{3}-M_{1}+2 M_{2}} .
\end{aligned}
$$

The description of this theory in terms of the Higgs branches presented above, which uses a classical analysis, make most sense when the couplings of the gauge theories are weak at the scale relevant for the superpotential. Let us call this scale $\Delta$ (simplifying the description by assuming that there is only one physical scale associated with the $W_{i}$. So the analysis above is valid if $\Lambda_{N_{i}}<<\Delta$. We then expect to get a gaugino condensation for the three remaining gauge groups $U\left(M_{i}\right)$ with scales given by $\Lambda_{M_{i}}<<\Delta$. The running of the coupling of various groups is depicted in Fig. 1. For scales $\mu>\Delta$ we have the $N=2$ running of the $U\left(N_{1}\right) \times U\left(N_{2}\right)$ and for scales $\mu<\Delta$ we have to take into account the superpotential and the Higgsing to the three $U\left(M_{i}\right)$ branches. Note that the couplings of the $U\left(M_{i}\right)$ groups at the scale $\Delta$ are given by $\frac{1}{g_{1}^{2}}, \frac{1}{g_{2}^{2}}, \frac{1}{g_{1}^{2}}+\frac{1}{g_{2}^{2}}$ which explains the values of the three coupling constant at $\mu=\Delta$ shown in Fig. 1. 


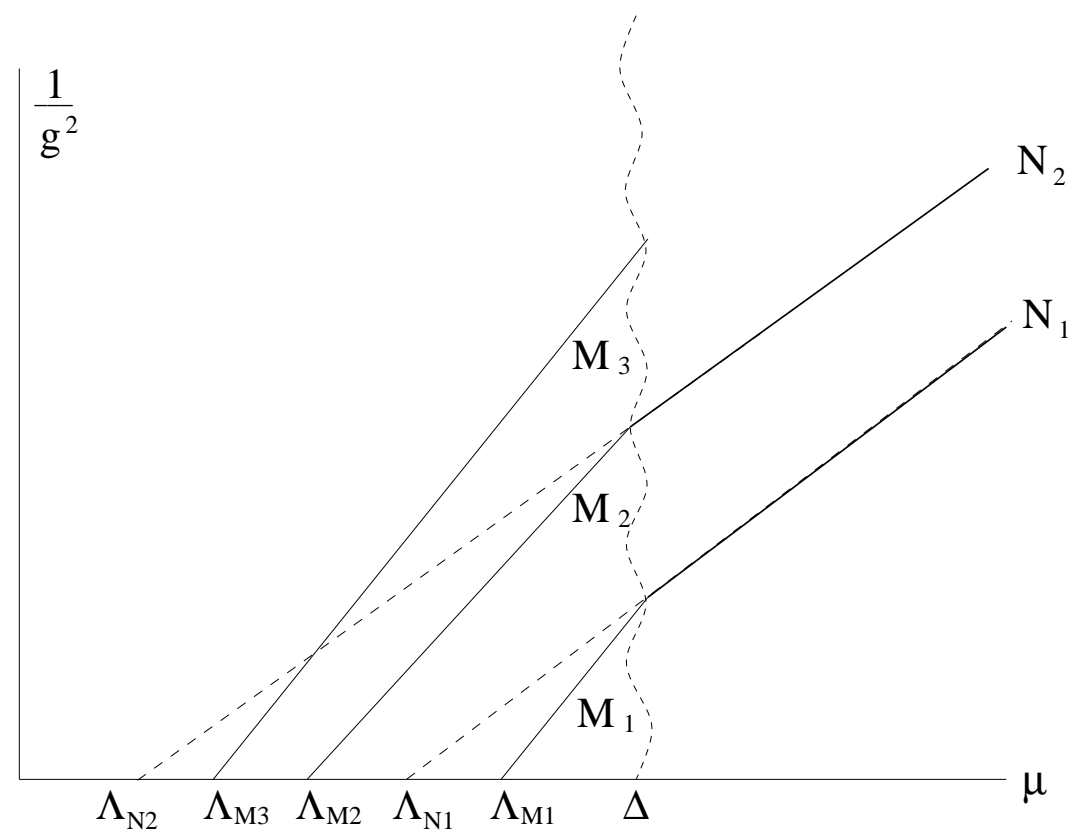

Figure 1:Running of gauge couplings for $\Lambda_{N_{1}} \sim \Lambda_{N_{2}} \ll \Delta$. Notice that $\frac{1}{g_{3}^{2}(\Delta)}=\frac{1}{g_{1}^{2}(\Delta)}+\frac{1}{g_{2}^{2}(\Delta)}$. The energy axis is plotted in logarithmic scale.

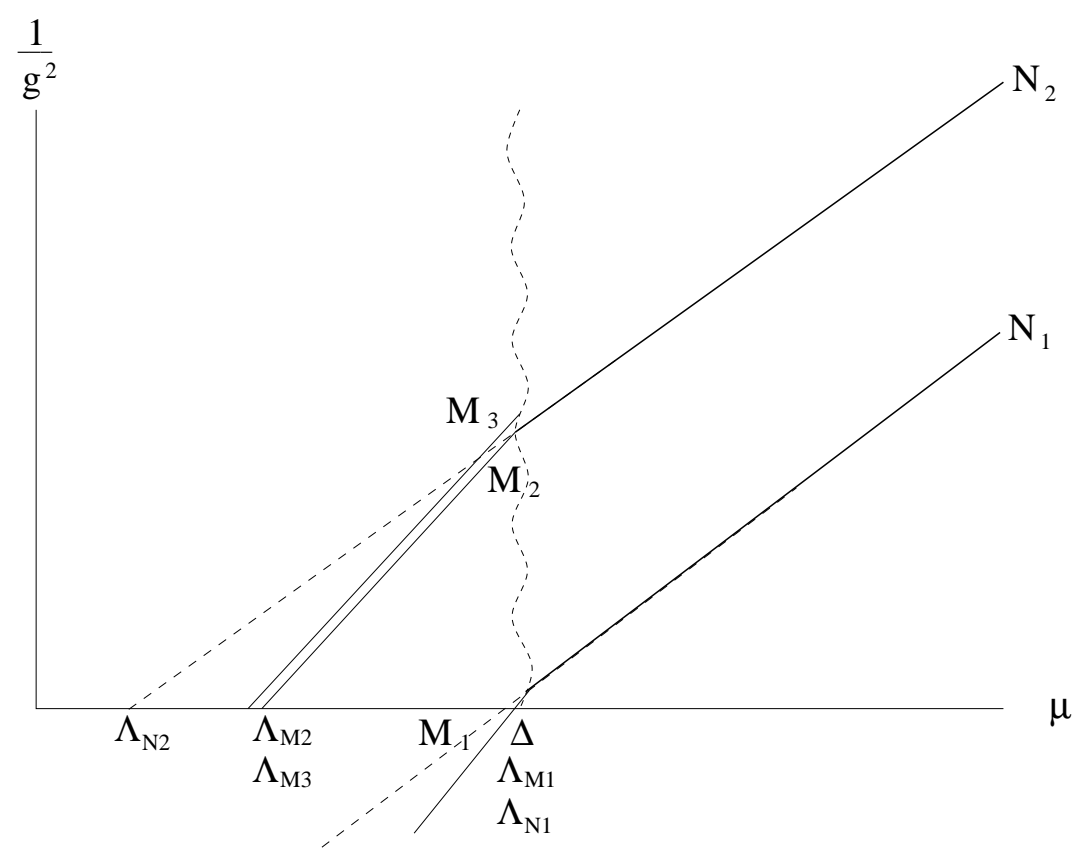

Figure 2:Running of gauge couplings for $\Lambda_{N_{1}} \sim \Delta, \Lambda_{N_{2}} \ll \Delta$. Notice that since $\frac{1}{g_{1}^{2}(\Delta)} \rightarrow 0$ one gets $\frac{1}{g_{3}^{2}(\Delta)}=\frac{1}{g_{2}^{2}(\Delta)}$.

When is it appropriate to dualize, say, $U\left(N_{1}\right) \rightarrow U\left(N_{2}-N_{1}\right)$ as in the previous section? Suppose e.g. that $N_{2}>N_{1}$. We start changing the scales of the theories such 
that $\Lambda_{N_{1}}$ becomes bigger, with $\Lambda_{N_{2}}$ held fixed. As we change $\Lambda_{N_{1}}$ we reach a point where $\Lambda_{N_{1}} \sim \Delta$. In this case we have the coupling constants given by Fig. 2 .

Now we ask what happens if we move $\Lambda_{N_{1}}$ to a region where $\Lambda_{N_{1}}>>\Delta$ ? The coupling $1 / g_{1}^{2}$ of the $U\left(N_{1}\right)$ group then formally goes to zero and becomes negative. It is then natural to use Seiberg duality to obtain a description in terms of a more weakly coupled theory with positive $1 / \widetilde{g}_{1}^{2}$. Note that now we are in a situation where $\Lambda_{N_{1}} \gg \Lambda_{N_{2}}$ so that, for energy scales $\Delta<\mu \leq \Lambda_{1}$, the $U\left(N_{1}\right)$ dynamics are important (with "negative $\left.1 / g_{1}^{2} "\right)$ and the $U\left(N_{2}\right)$ can effectively be treated as a spectator flavor symmetry, which is weakly gauged. Thus we replace $U\left(N_{1}\right) \times U\left(N_{2}\right)$ with its dual gauge group $U\left(\widetilde{N}_{1}\right) \times U\left(\widetilde{N}_{2}\right)$ where $\widetilde{N}_{1}=N_{2}-N_{1}$ and $\widetilde{N}_{2}=N_{2}$, with $W_{1} \rightarrow-W_{1}$ and $W_{2} \rightarrow W_{1}+W_{2}$ and $\frac{1}{g_{1}^{2}}=-\frac{1}{g_{1}^{2}}$ and $\frac{1}{\widetilde{g}_{2}^{2}}=\frac{1}{g_{2}^{2}}+\frac{1}{g_{1}^{2}}$. However this cannot be a good description if $M_{1} \neq 0$; in this case (as an approximate relation) we have $\Lambda_{M_{1}} \sim \Lambda_{N_{1}}$ so the good description of the theory for scales $\mu<\Lambda_{N_{1}}$ should include the gaugino condensation (and various corrections associated with $W)$ in an $\mathcal{N}=1$ factor. Thus, for $M_{1} \neq 0$, once we go to scales $\mu<\Delta$ we will encounter strong coupling quantum corrections (involving gaugino condensation etc.) and thus we do not have a weak coupling classical description. We now consider the branch where $M_{1}=0$. Then $\Lambda_{M_{1}}=0$ and there is no gaugino condensate associated with that branch. Thus we will have the running of the couplings depicted in the figure below:

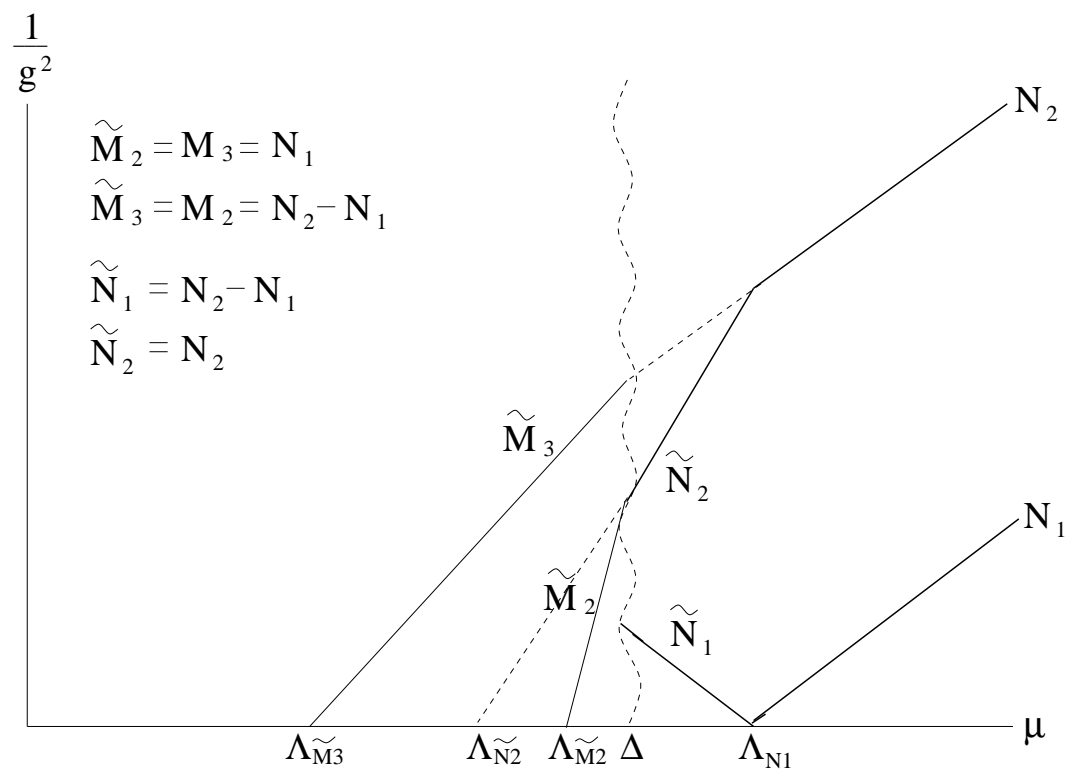

Figure 3:Running of gauge couplings. Notice that at $\mu=\Lambda_{N_{1}}$ a Seiberg duality is necessary to keep $\frac{1}{g_{1}^{2}(\mu)}>0$ for $\mu<\Lambda_{N_{1}}$. 
Note that in this case after we hit the scale $\mu=\Delta$ we will Higgs the theory to $U\left(\widetilde{M}_{2}\right) \times U\left(\widetilde{M}_{3}\right)\left(\right.$ with $\widetilde{M}_{3}=\widetilde{N}_{1}=N_{2}-N_{1}$ and $\widetilde{M}_{2}=\widetilde{N}_{2}-\widetilde{N}_{1}=N_{1}$, after which the theory undergoes gaugino condensate in each factor. Note however in terms of the original assignments of branches what we had called $M_{2}, M_{3}$ have switched roles: $\widetilde{M}_{2}=M_{3}$ and $\widetilde{M}_{3}=M_{2}$. This is exactly the action of the Weyl group generated by $e_{1}$ on the corresponding roots. Note that this is consistent with the field theory analysis for scales as well. According to (8.8), after the duality $\Lambda_{\widetilde{N}_{1}}=\Lambda_{N_{1}}$ and $\Lambda_{\widetilde{N}_{2}}^{2 N_{2}-\widetilde{N}_{1}}=\Lambda_{N_{2}}^{2 N_{2}-N_{1}} \Lambda_{N_{1}}^{2 N_{1}-N_{2}}$. We see from (9.3) that for $\widetilde{M}_{1}=M_{1}=0$ the duality maps $\Lambda_{\widetilde{M}_{2}}=\Lambda_{M_{3}}$ and $\Lambda_{\widetilde{M}_{3}}=\Lambda_{M_{2}}$. The $M_{1}=\widetilde{M}_{1}=0$ branch corresponds, in terms of the discussion at the end of sect. 8.1, to the vacua which are semi-classically visible in the dual $U\left(N_{f}-N_{c}\right)$ theory, with unbroken $U\left(N_{f}\right)$.

\subsection{Geometry}

Let us describe the same case from the geometry side. We will first present qualitatively how the geometry analysis works. However we will be able to do more, namely we will provide also a basis for an exact quantitative analysis of the vacuum structure of the theory.

Geometrically over the $t$ plane we have three double points where the three $S^{3}$ 's will emerge with sizes given by $\Lambda_{M_{i}}$, separated by distance of the order of $\Delta$. In the limit $\Lambda_{M_{i}}<<\Delta$ we have three small $S^{3}$ 's. Now we analyze the theory with respect to scales: For very high energies $\mu>>\Delta$, this translates to probing the geometry at $t \sim \mu>>0$ (using the holographic picture) which basically means that the sizes of $S^{3}$ 's and the scale of $W$ is negligible and we have the $\mathcal{N}=2$ description. This means far away from the "tips" of the cone we have a description of the geometry in terms of the $S^{2}$ 's with normal bundle which is essentially $\mathcal{O}(-2) \oplus \mathcal{O}(0)$ with varying $B$-field which gives the variation of $1 / g_{i}^{2}$. This in turn can be captured by $\Delta\left(1 / g^{2}(t)\right)=\int_{t}^{\Lambda_{0}} H$ which gives the expected running. As we approach the scales $t=\mu \sim \Delta$ this description breaks down and we can distinguish the three distinct points which correspond to blow downs of the three $S^{2}$ 's corresponding to three branches. Now we see the corresponding $S^{2}$ 's and their running in accordance with $\mathcal{N}=1$ running of the couplings. As we approach each double point we begin to see that each double point has led to an $S^{3}$ which is identified with the gaugino condensation in the gauge theory. This is assuming all the $M_{i} \neq 0$. If any of the $M_{i}=0$ the corresponding $S^{3}$ does not emerge and has vanishing size as there is no flux to support the $S^{3}$ (which is dual to the statement that there is no gaugino condensate if there is no gauge group!) 
If we now consider increasing the scale of $\Lambda_{N_{1}}$, if none of the $M_{i}$ are zero, this makes the size of $S^{3}$ corresponding to $M_{1}$ to become larger. Now suppose $\Lambda_{N_{1}}>\Delta$. In this case when we consider the description of the $B$ field as we come towards the tip of the cone we see that the corresponding $S^{2} \rightarrow 0$. However this happens at a scale where the $S^{3}$ corresponding to branch one is large. Thus as we approach closer to the tip of the geometry we do not have any gauge theoretic description, as the gaugino condensation in the first factor would have already taken place. However, suppose $M_{1}=0$. In this case the size of the $S^{3}$ corresponding to the first branch remains vanishing as we change $\Lambda_{N_{1}}$ even after we pass to $\Lambda_{N_{1}}>\Delta$. In this case when we describe the geometry as we approach the tips of the cone as we get to the scale $t \sim \Lambda_{N_{1}}$ we have the $S^{2} \rightarrow S^{2}$ transition giving us a dual geometry. This is still a good description because the $S^{3}$ 's are much smaller and we can ignore them, even as we approach $t \sim \Delta$. As we go towards the tips of the cone we find two $S^{3}$ 's with finite size, which is the scale the gaugino condensates take place. In terms of the new branches, the role of the two $S^{3}$ 's have switched in accordance with the Weyl reflection.

This was a qualitative description of how the geometry sees the gauge theory description given above. However the geometry also yields precise quantitative information which we now discuss. Before turning to the $X\left(1, A_{2}\right)$ example let us start by illustrating the deformation procedure of section 6 corresponding to the large $\mathrm{N}$ dual for $X\left(k, A_{r}\right)$ and then we set $r=2$ and $k=1$.

Recall the equation for the $A_{r}$ ALE space fibered over the t-plane given by (6.1), where the fibration data and tree level superpotentials are related explicitly by (6.3),

$$
W_{i}^{\prime}(t)=t_{i}(t)-t_{i+1}(t) \quad \text { for } \quad i=1, \ldots, r \quad \text { with } \quad \sum_{i=1}^{r+1} t_{i}(t)=0
$$

The charges of $x, y, z$ and $t$ are

$$
Q(x)=\frac{1}{2} \quad Q(y)=\frac{1}{2} \quad Q(z)=\frac{1}{r+1} \quad Q(t)=\frac{1}{k(r+1)}
$$

giving for the central charge (in agreement with (6.8) for $C_{2}\left(A_{r}\right)=r+1$ )

$$
\hat{c}=(1-2 Q(x))+(1-2 Q(y))+(1-2 Q(z))+(1-2 Q(t))=2 \frac{(r k-1)}{k(r+1)} .
$$

The $(\log )$ normalizable deformations are easily computed, by looking for monomials with charges (equal to) less than $\frac{\hat{c}}{2}$, to be,

$$
P_{k-1}(t) z^{r-1}+P_{2 k-1}(t) z^{r-2}+\ldots+P_{(r-1) k-1}(t) z+P_{r k-1}(t)
$$


where $P_{j}(t)$ are polynomials of degree $j$ in $t$. The number of deformation parameters is then given by $k \sum_{j=1}^{r} j=k \frac{r(r+1)}{2}$. This is the same as $k R_{+}$as expected. Moreover, the $\log$ normalizable ones correspond to the leading coefficient of each $P_{j}$ and there are exactly $r$ of them.

Let us now specialize to $X\left(1, A_{2}\right)$. The deformed geometry corresponds to,

$$
x^{2}+y^{2}+\left(z+t_{1}(t)\right)\left(z+t_{2}(t)\right)\left(z+t_{3}(t)\right)+a z+b t+c=0
$$

where $a z$ and $b t$ are $\log$ normalizable deformations and $c$ is normalizable. From the tree level superpotentials (9.1), the fibration data is given as,

$t_{1}(t)=\frac{1}{3}\left(2 W_{1}^{\prime}(t)+W_{2}^{\prime}(t)\right) \quad t_{2}(t)=\frac{1}{3}\left(-W_{1}^{\prime}(t)+W_{2}^{\prime}(t)\right) \quad t_{3}(t)=-\frac{1}{3}\left(W_{1}^{\prime}(t)+2 W_{2}^{\prime}(t)\right)$

For zero deformation parameters, i.e., $a=b=c=0$ there are three singular points in the geometry, given by the location of double roots of the discriminant of the cubic equation in $z$ for $x=y=0$. The discriminant is given by,

$$
\Delta=\left(t_{1}(t)-t_{2}(t)\right)^{2}\left(t_{2}(t)-t_{3}(t)\right)^{2}\left(t_{3}(t)-t_{1}(t)\right)^{2}
$$

The solutions of $\Delta=0$ are then given by solving,

$t_{1}(t)-t_{2}(t)=W_{1}^{\prime}(t)=0 \quad t_{2}(t)-t_{3}(t)=W_{2}^{\prime}(t)=0 \quad$ and $\quad t_{1}(t)-t_{3}(t)=W_{1}^{\prime}(t)+W_{2}^{\prime}(t)=0$

This makes explicit the 1-1 correspondence of $S^{3}$ 's with the positive roots of $A_{2}$ : after tuning on $a, b$ and $c$, the geometry is smooth, since each double root of $\Delta$ splits into two, giving rise to an $S^{3}$ of non-zero volume. Let us write $\Delta$ as,

$$
\Delta=\left(t-a_{1}^{+}\right)\left(t-a_{1}^{-}\right)\left(t-a_{2}^{+}\right)\left(t-a_{2}^{-}\right)\left(t-a_{3}^{+}\right)\left(t-a_{3}^{-}\right)
$$

If we write (9.4) as,

$$
x^{2}+y^{2}+\left(z-z_{1}(t)\right)\left(z-z_{2}(t)\right)\left(z-z_{3}(t)\right)=0
$$

then the compact periods of the holomorphic three form $\Omega$ are given by,

$$
S_{K}=\frac{1}{2 \pi i} \int_{a_{K}^{-}}^{a_{K}^{-}}\left(z_{I}(t)-z_{J}(t)\right) d t \quad \text { for } \quad(K, I, J) \quad \text { cyclic permutations of }
$$


and non-compact periods by,

$$
\Pi_{K}=\frac{1}{2 \pi i} \int_{a_{K}^{+}}^{\Lambda_{0}}\left(z_{I}(t)-z_{J}(t)\right) d t
$$

with $(K, I, J)$ as before.

The superpotential (6.11) is in this case,

$$
\frac{1}{2 \pi i} W_{\mathrm{eff}}=M_{1} \Pi_{1}+M_{2} \Pi_{2}+M_{3} \Pi_{3}+\frac{\alpha_{1}}{2 \pi i} S_{1}+\frac{\alpha_{2}}{2 \pi i} S_{2}+\frac{\alpha_{3}}{2 \pi i} S_{3}
$$

with $\alpha_{3}=\alpha_{1}+\alpha_{2}$,

$$
\alpha_{1}=\frac{1}{g_{1}^{2}\left(\Lambda_{0}\right)}=\left(2 N_{1}-N_{2}\right) \log \left(\frac{\Lambda_{N_{1}}}{\Lambda_{0}}\right) \quad \text { and } \quad \alpha_{2}=\frac{1}{g_{2}^{2}\left(\Lambda_{0}\right)}=\left(2 N_{2}-N_{1}\right) \log \left(\frac{\Lambda_{N_{2}}}{\Lambda_{0}}\right)
$$

Let us describe the two interesting cases given in Figure 1 and Figure 3. The first corresponds to the case where $\Lambda_{N_{1}}$ and $\Lambda_{N_{2}}$ are much smaller than $m_{1}, m_{2}, a_{1}-a_{2}, a_{2}-a_{3}$ and $a_{1}-a_{3}$.

As discussed for general $X(k, G)$ in section 6 , if the two theories are asymptotically free, i.e, $2 N_{2}-N_{1}>0$ and $2 N_{1}-N_{2}>0$, then the exact superpotential admits an expansion of the form,

$$
W_{\mathrm{eff}}=\sum_{K=1}^{3} M_{K}\left(S_{K} \log \left(\frac{\Lambda_{K}^{3}}{S_{K}}\right)+\sum_{n, m, p=1}^{\infty} h_{n m p}^{K} S_{1}^{n} S_{2}^{m} S_{3}^{p}\right)
$$

where $h_{n m p}^{K}$ only depends on the classical superpotential parameters, but not on the particular Higgsing, and $\Lambda_{K}$ 's are the scales from the threshold matching conditions. This also yields the running of the coupling constants as depicted in the figure 1.

The second interesting case is when $\Lambda_{N_{1}}$ is taken to be larger than the scales set by the superpotentials keeping $\Lambda_{N_{2}}$ fixed as before. For $M_{1}>0$, this limit implies that $S_{1}$ grows indefinitely and no weak coupling description is available anymore. However, for $M_{1}=0$, as we will now argue there is a vanishing $S^{3}$ corresponding to that branch. At a leading order this is obvious, because the gaugino condensate analysis suggests $S_{1} \sim e^{-1 / g^{2} M_{1}}$ which as $M_{1} \rightarrow 0^{+}$gives $S_{1} \rightarrow 0$. However, in order to argue that this also continues to be the case even when $\Lambda_{N_{1}}$ gets bigger we need to show that in an analytic expansion $S$ is still zero. 
The weak coupling approximation leads to an expression for $S_{1}$ in terms of a power expansion with no order zero term in,

$$
T=\left(\frac{\Lambda_{N_{1}}}{\Delta}\right)^{\frac{2 N_{1}-N_{2}}{M_{1}}}
$$

In the limit $M_{1} \rightarrow 0^{+}, T \rightarrow 0$ since $\frac{\Lambda_{N_{1}}}{\Delta}<1$ and $2 N_{1}-N_{2}>0$. Thus we have $S_{1}=0$ in an analytic neighborhood, which by analytic continuation implies its vanishing for all values of $\Lambda_{N_{1}}$. In this situation the weak coupling description for the $S_{2}, S_{3}$ will still be valid. Here as we increase $\Lambda_{N_{1}}$ we encounter the situation shown in Fig. 3 where the couplings run until we get to a situation where we have to describe it in terms of a dual description. Here since the $S_{2}, S_{3}$ are small and $S_{1}$ is vanishing we can still continue pretending to be in the phase where the $S^{2}$ 's are blown up with a dual description. This is just the same geometry, of course, but interpreted differently.

In order to see explicitly this description notice that the same geometry with the same units of fluxes can be interpreted in a different way by the following simple identification,

$$
z_{1}(t)=\widetilde{z}_{2}(t) \quad z_{2}(t)=\widetilde{z}_{1}(t) \quad z_{3}(t)=\widetilde{z}_{3}(t)
$$

In terms of the new identification we are led to write the superpotential corresponding to this geometry and fluxes as follows,

$$
\frac{1}{2 \pi i} W_{\text {eff }}=M_{3} \widetilde{\Pi}_{2}+M_{2} \widetilde{\Pi}_{3}-\frac{\alpha_{1}}{2 \pi i} \widetilde{S}_{1}+\frac{\alpha_{3}}{2 \pi i} \widetilde{S}_{2}+\frac{\alpha_{2}}{2 \pi i} \widetilde{S}_{3}
$$

with the following identification:

$$
\widetilde{M}_{1}=0 \quad \widetilde{M}_{2}=M_{3} \quad \widetilde{M}_{3}=M_{2} \quad \widetilde{\alpha}_{1}=-\alpha_{1} \quad \widetilde{\alpha}_{2}=\alpha_{3} \quad \widetilde{\alpha}_{3}=\alpha_{2}
$$

This dual description arises as the IR of an $U\left(\tilde{N}_{1}\right) \times U\left(\tilde{N}_{2}\right) \mathcal{N}=2$ gauge theory with superpotentials,

$$
\widetilde{W_{1}^{\prime}}(t)=-W_{1}^{\prime}(t) \quad \widetilde{W_{2}^{\prime}}(t)=W_{1}^{\prime}(t)+W_{2}^{\prime}(t)
$$

where,

$$
\widetilde{N}_{1}=\widetilde{M}_{1}+\widetilde{M}_{3}=N_{2}-N_{1} \quad \widetilde{N}_{2}=\widetilde{M}_{2}+\widetilde{M}_{3}=N_{2}
$$

As discussed in section 6 , the original weak gauge theory description is invalid because $U\left(N_{1}\right)$ is asymptotically free and $\Lambda_{N_{1}}>\Delta$. On the other hand, the dual theory also has $\widetilde{\Lambda}_{\widetilde{N}_{1}}=\Lambda_{N_{1}}>\Delta$, however, $U\left(\widetilde{N}_{1}\right)$ is now IR free, and the proof of validity of the weak coupling description is valid.

As we approach scales given by $S_{2}, S_{3}$ the weak coupling gauge theory description ceases to make sense and we have the geometry involving the two blown up $S^{3}$ 's. 


\section{Affine A-D-E quivers, RG cascades and affine Weyl reflections}

Up to now, as far as the quantum analysis, we have mainly concentrated on the nonaffine case. In this section we will discuss aspects of affine quiver theories. We will also indicate the relation between affine case and the non-affine case. In fact we will motivate the discussion of the affine case from this viewpoint.

Consider a non-affine case we have studied. Suppose we are in a situation where the ranks $N_{i}$ are such that all the $\mathcal{N}=2$ quiver gauge groups are asymptotically free. Let us discuss the theory at scales $\mu>>\Delta$ where $\Delta$ denotes the scales relevant for the superpotentials. In this situation the couplings get weaker and weaker as we go backwards towards the UV. However, it is crucial to remember that these field theories were obtained from a decoupling limit of some string theory and even though $\frac{1}{g_{s}}$ has been taken to be much larger than any of the inverse square gauge couplings, eventually those will become comparable to $\frac{1}{g_{s}}$ in the UV. This implies that stringy modes are not negligible and the field theory description in terms of the non-affine quiver theory is inadequate. However we can view this as a special case of the affine quiver theory, and we know that a weighted sum of the inverse coupling constants squared is $1 / g_{s}$. This means that at some point we can get a situation where $1 / g_{0}^{2}<0$, i.e. the node associated with the affine extension would have a negative coupling. Formally we may not be bothered by this because there is no brane wrapping it before. However, if we wish that the string coupling be weaker than all the other couplings we will have to do an inverse duality of the form corresponding to the case $N_{f}=N_{c}$, corresponding to a Weyl reflection on the affine node. Continuing this towards the UV we will end up, as we will discuss below with the inverse RG cascade. Of course the infrared physics does not get modified from the non-affine case and so our discussion of the superpotential etc. for the non-affine case would still be valid. Note however that there are other affine cases which do not end up as a UV completion of non-affine case. In particular, the projection of branches for the positive affine roots to non-affine roots, leads to both positive and negative roots. This implies that in the affine case we will end up with the superpotential where $M_{i}$ are the net number of branches for a given positive root, which thus can be a positive or negative integer. This is the only new ingredient in the context of affine case as far as the superpotential analysis is concerned.

In the remainder of this section we will explain how the RG cascade arises in the affine case and its relation to affine Weyl group. 


\subsection{RG cascade and affine quivers}

Consider the general $\mathcal{N}=1$ affine quiver theory, with $W_{i}=\frac{s_{i}}{k+1} \operatorname{Tr} \phi_{i}^{k+1}$, and gauge group $\prod_{i=0}^{r} U\left(\hat{N}_{i}\right)$, with $\hat{N}_{i}=N_{0} d_{i}+N_{i}$, for general $N_{i}$ with $\hat{N}_{0}=N_{0}$. In the AdS/CFT correspondence, the $N_{i} \neq 0$ correspond to introducing wrapped branes. If all $N_{i}=0$, the theory could be scale invariant. For some $N_{i} \neq 0$, some of the groups are asymptotically free and others are IR free. The gauge couplings of the asymptotically free groups increase in the IR until, eventually, it becomes appropriate to dualize the group. This leads to a RG cascade, via the various affine Weyl reflections, which generalizes the case $\widehat{A}_{1}$, with $k=1$, found in [12].

The cascading effectively reduces $N_{0}$. But nothing holomorphic depends on $N_{0}$. For example, the holomorphic beta functions (4.5) for $\hat{N}_{i}=N_{0} d_{i}+N_{i}$ are independent of $N_{0}$ since $d_{i}$ is a null vector of $C_{i j}$. Also, though the theory is not conformal when the $N_{i} \neq 0$, the holomorphic beta function for the diagonally embedded $U\left(N_{0}\right)_{D}$, whose gauge coupling (4.4) is given by the string coupling, always vanishes

$$
\beta_{D} \equiv \sum_{i=0}^{r} d_{i} \beta_{i}=0
$$

One might wonder whether or not similar statements apply for the physical beta functions, e.g. the physical beta functions (4.7) will be independent of $N_{0}$ as long as all $\gamma\left(\phi_{i}\right)$ are equal. Likewise the physical beta functions will satisfy 10.1 if $\beta\left(\lambda_{i j}\right)=0$ and all $\gamma\left(\phi_{i}\right)$ are equal. These statements should indeed be true in the limit where $N_{0}$ is large compared to the $N_{i}$, i.e. away from the IR limit where $N_{0}$ has cascaded away. But in the far IR this description is no longer even useful, as the theory breaks up into the vacuum branches, with gaugino condensation, discussed in the earlier sections. We'll discuss the RG flow as the theory cascades, above the scale where it eventually confines.

As already discussed, we parameterize the gauge couplings as

$$
g_{i}^{-2}(\vec{x})=\frac{1}{g_{s}} \delta_{i, 0}+\vec{e}_{i} \cdot \vec{x}
$$

with $\vec{x}$ in the $\widehat{G}$ Coxeter box, defined by the condition that all $g_{i}^{-2}(\vec{x}) \geq 0$. The vanishing of the beta function (10.1) corresponds to the fact that (10.2) gives

$$
g_{D}^{-2} \equiv \sum_{i=0}^{r} d_{i} g_{i}^{-2}(\vec{x})=\frac{1}{g_{s}}
$$


independent of $\vec{x}$. In the AdS/CFT correspondence, for large $N_{0}$, we can also see this equality (10.3), and the statement (10.1) corresponds to the fact that, even with the wrapped branes, the solution has constant dilaton.

Now we write the beta functions for the couplings (10.2) as $\beta_{i}=-8 \pi^{2} \dot{g}_{i}^{-2}$, with $\cdot \equiv-d / d(\ln \mu)$. Using (10.2), we get

$$
\beta_{i}=-8 \pi^{2} \vec{e}_{i} \cdot \dot{\vec{x}} \quad \text { and thus } \quad-8 \pi^{2} \dot{\vec{x}}=\vec{N}
$$

where 'represents the flow towards IR, and we have used (4.2). Think of $\vec{x}$ as the position of a particle living in the Coxeter box; according to (10.2), this position gives the gauge couplings. The particle is moving with a velocity $\dot{\vec{x}}$, which corresponds to the beta functions as in (10.4).

In fact, we can make a more complete mechanical analogy. The walls of the Coxeter box are in one-to-one correspondence with the simple roots $\vec{e}_{i}$, since $\vec{e}_{i}$ is the normal vector to the wall where $g_{i}^{-2}(\vec{x})=0$. Label the wall associated with $e_{i}$ by $i$. Consider attaching the particle to the $i$-wall of the Coxeter box by $N_{i}$ strings of equal tension. For a fixed position of the particle in the Coxeter box the lowest energy state for any such configuration arises when the strings are perpendicular to the walls. The $U\left(N_{i}\right)$ coupling constant $g_{i}^{-2}(\vec{x})$ is identified with the length of the corresponding string (i.e. the distance of the particle to the $i$-th wall). See Fig. 4B. It is an easy exercise to see that if $N_{i}=N_{0} d_{i}$ there is no net force on the particle due to the strings. This is the case when the beta function vanishes and $\dot{\vec{x}}=0$. If $N_{i} \neq N_{0} d_{i}$ there would be a net force. Noting that the direction of the $N_{i}$ string is in the $-\vec{e}_{i}$ direction (as that is a vector perpendicular to the corresponding wall and in the correct orientation) we see that the net force is given by (choosing the tension to be $1 / 8 \pi^{2}$ )

$$
\vec{F}=-\frac{1}{8 \pi^{2}} N_{i} \vec{e}_{i}=-\frac{1}{8 \pi^{2}} \vec{N}
$$

Now, using (10.4) we see that

$$
\dot{\vec{x}}=\vec{F}
$$

and this captures the RG flow; this is not quite the Newton's equation, as the force is giving the velocity. The similarity of this to brane constructions of gauge theory is striking. In fact at least in one case (which we discuss in section 12) it is identical to one. 


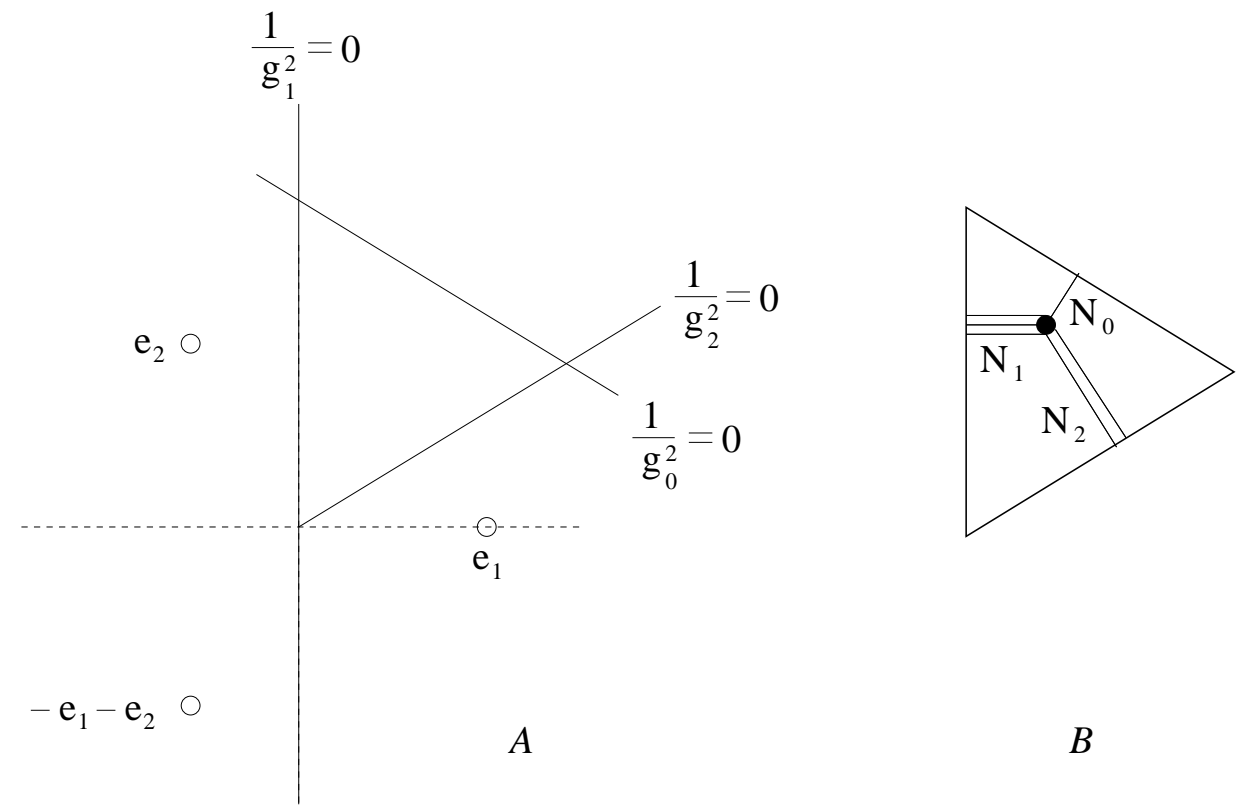

Figure 4:A) Construction of the Coxeter Box for $\hat{A}_{2} . \vec{e}_{1}$ and $\vec{e}_{2}$ are the simple roots of $A_{2} . B$ ) Particle living in the Coxeter box attached to the walls where $\frac{1}{g_{i}^{2}}=0$ by $N_{i}$ strings.

If there are wrapped branes and the theory is not conformal, the particle moves in the direction of $\vec{F}$ until eventually it hits one of the walls of the Coxeter box, which is defined where one of the $g_{i_{0}}^{-2}$ given by (10.4) vanishes. At this wall the gauge group $U\left(\hat{N}_{i_{0}}\right)$ gets strong and should be dualized. As discussed earlier, this duality corresponds to a Weyl reflection. It is precisely a Weyl reflection about the corresponding wall of the Coxeter box.

In other words, the particle bounces off the wall, with angle of incidence equal to angle of reflection. This can be seen from the beta function transformation (8.5), which gives

$$
\vec{e}_{i} \cdot \dot{\vec{x}}^{\prime}=\vec{e}_{i} \cdot \dot{\vec{x}}-\left(\vec{e}_{i} \cdot \vec{e}_{i_{0}}\right)\left(\dot{\vec{x}} \cdot \vec{e}_{i_{0}}\right)
$$

with $\dot{\vec{x}}^{\prime}$ the particle's velocity after bouncing off the wall of the Coxeter box. We thus obtain

$$
\dot{\vec{x}}^{\prime}=\dot{\vec{x}}-\vec{e}_{i_{0}}\left(\dot{\vec{x}} \cdot \vec{e}_{i_{0}}\right)
$$

which is precisely a Weyl reflection of the velocity $\dot{\vec{x}}$, reversing the component normal to the wall and preserving the component parallel. So the particle bounces off the walls with angle of incidence equal to angle of reflection.

Of course, which wall the particle hits depends on both its velocity and its initial position. The initial position of the particle is given by the scales $\Lambda_{i}$ of the $U\left(\hat{N}_{i}\right)$ groups, 
as these are the integration constants in integrating the beta function equations. Suppose that we integrate the 1-loop beta functions as

$$
g_{i}^{-2}(\mu)=\frac{\beta_{i}}{8 \pi^{2}} \ln \left(\frac{\mu}{\Lambda_{i}}\right)
$$

where the $g_{s}^{-1} \delta_{i, 0}$ term in $(\sqrt[10.2)]{)}$ can be thought of as scaled into $\Lambda_{0}$. In order to have all $g_{i}^{-2} \geq 0$, the scales should satisfy the condition (8.10) which, as discussed in sect. 8, is properly preserved upon dualizing.

If we flow to the IR, decreasing $\mu$, the first group $i_{0}$ to be dualized is that whose scale $\Lambda_{i_{0}}$ which $\mu$ hits first. After the duality transformation, the scales are modified as in (8.8). The condition which led to (8.8) is precisely equivalent to the condition that the particle's position $\vec{x}$ is continuous across the bounce, even though the velocity is reflected. The picture of the bouncing particle captures the details of the RG flow in a simple fashion.

For $\widehat{A}_{1}$, the Coxeter box is the interval $0 \leq x \leq g_{s}^{-1}$ and the cascade of [12] (which was the $k=1$ case, but the present picture applies for all $k$ ), corresponds to the particle bouncing back and forth in this interval. For the $\widehat{A}_{2}$ case the Coxeter box is the inside of an equilateral triangle (see Fig. $4 \mathrm{~A}$ ) and for the $\widehat{A}_{3}$ case it's the inside of a tetrahedron. For generic velocity, the particle will bounce off of each of the $r+1$ walls in succession.

In all cases, the cascading reduces the number $N_{0}$ of units of $F_{5}$ flux. As discussed before (8.3), after bouncing off of the wall where $g_{i_{0}}^{-2}=0$, the $\hat{N}_{i}$ change according to (8.2); note that $\hat{N}_{i_{0}}^{\prime}=\hat{N}_{i_{0}}-\sum_{j} C_{i_{0} j} \hat{N}_{j}=N_{0} d_{i_{0}}-\sum_{j} C_{i_{0} j} N_{j}$. The number of units of $N_{0}$ charge is also reduced in correspondence with the root lattice translations $T$ in the affine Weyl group $\widehat{W} \cong W \ltimes T$. More precisely suppose we start in the UV with a quiver theory with $U\left(N_{i}\right)$ as $i=1, \ldots, r+1$. As we go towards the IR we undergo Weyl reflections to stay in the Coxeter box. However suppose we continued the line not worrying about staying in the Coxter box (i.e. use the variables of the original gauge theory even passed the negative coupling squared). After a while we can bring the particle back to the fundamental domain given by the Coxeter box by a translation vector $\vec{R}$ in the root lattice. This would be of course in the direction of $-\vec{N}$ and so let us write it as $\vec{R}=-a \vec{N}$ with $a>0$. The change in the 3-brane charge is given by

$$
\Delta N_{0}=\sum_{i} N_{i} \vec{e}_{i} \cdot \vec{R}=-a \vec{N} \cdot \vec{N}
$$

This follows, just as in the case [12] 48 from the fact that

$$
\Delta N_{0}=8 \pi^{2} \int H_{N S} \wedge H_{R}
$$


and using the fact that $H_{R}$ is characterized by the vector $\sum_{i} N_{i} \vec{e}_{i}$ and $\int H_{N S}$ which is the vector $\Delta \frac{1}{g_{i}^{2}}$ is given by the vector $\vec{R}$. Note that in this way it is clear the $\Delta N_{0}<0$ as we proceed towards IR. Thus cascade phenomenon is directly related to the translation part of the affine Weyl group. Also note that the above formula for the change of the threebrane charge is also valid continuously as we continuously change $a$ because $a=\log \left(\mu_{1} / \mu_{2}\right)$ as we change the scale from $\mu_{1}$ to $\mu_{2}<\mu_{1}$.

\section{Affine $\mathcal{N}=1$ theories: the conformal case}

Consider the theory of 3-branes in the presence of a point-like singularity of the Calabi-Yau. One expects to get a conformal theory. For example considering $N_{0} 3$-branes at the conifold singularity 23] it was argued that one gets a conformal theory, with a natural AdS dual description. In general this is expected from the geometry if the singular limit admits a smooth deformation with no 2-cycles left. In this case there are no allowed instantons and so there are no quantum corrections to the geometry. Thus also in the limit that the deformation disappears one expects to have no quantum deformations for the singular classical geometry. Typically for probes in the presence of singularities protected by quantum corrections one expects to obtain conformal theories.

Let us now consider a class of examples which yield singular 3-folds, where singularity is at a point. Of course all the geometries we considered admit deformations which have no 2-cycles, and so the singular limit is expected to be a good description also quantum mechanically. Consider taking all

$$
W_{i}\left(\phi_{i}\right)=\frac{s_{i}}{k+1} \operatorname{Tr} \phi_{i}^{k+1}
$$

homogeneous. Then it is possible to see using our geometric description that we have a local description of Calabi-Yau which has an isolated singularity at the origin given by a quasi-homogeneous hypersurface in $\mathbf{C}^{4}$. Thus we expect in all such cases that the 3 -brane probe to yield $\mathcal{N}=1$ conformal theories. Note that not all 3 -fold point-like singularities with 3-brane probes yield $\mathcal{N}=1$ superconformal gauge theories. For example as discussed in [17] the threebranes in the presence of $x^{2}+y^{2}+z^{2}+w^{l}=0$ even though it is expected to be conformal, has no conventional gauge theoretic description for $l$ odd (for $l=2 k$ it corresponds to affine $A_{1}$ theory with superpotential $\left.\Phi^{k+1}\right)$. 
We now provide evidence for the existence of the conformal fixed point for this class of quiver theories from the gauge theory analysis. The exact beta function for the $s_{i}$ is

$$
\beta\left(s_{i}\right)=-3+(k+1) \Delta\left(\phi_{i}\right) \equiv-3+(k+1)\left(1+\frac{1}{2} \gamma\left(\phi_{i}\right)\right) .
$$

The superpotential respects a classical $U(1)_{R}$ symmetry under which

$$
R(\phi)=\frac{2}{k+1} \quad R\left(Q_{i j}\right)=\frac{k}{k+1}
$$

and the gauginos have charge +1 . Generally this $U(1)_{R}$ symmetry is anomalous, which is the case if any $U\left(N_{i}\right)$ instanton 't Hooft vertex has non-zero $U(1)_{R}$ charge. The total $U(1)_{R}$ charge of the $U\left(N_{i}\right)$ instanton 't Hooft vertex is

$$
R\left(\Lambda_{i}^{\vec{e}_{i} \cdot \vec{N}}\right)=\frac{2}{k+1}\left(\vec{e}_{i} \cdot \vec{N}\right)
$$

with $\vec{N}=\sum_{i=0}^{r} \vec{e}_{i} N_{i}$. These are only all zero if the diagram is the affine $\widehat{G}$ diagram and $N_{i}=N_{0} d_{i}$, with $N_{0}$ arbitrary. More generally, though some of the (11.4) are non-zero, the 't Hooft vertices could be invariant under a discrete subgroup of this $U(1)_{R}$, corresponding to an anomaly free discrete R-symmetry.

The $\widehat{G}$ affine quiver theories with $N_{i}=N_{0} d_{i}$ thus satisfy the necessary conditions for a $\mathcal{N}=1$ superconformal field theory: there is an anomaly free $U(1)_{R}$ symmetry, which is needed for the superconformal current multiplet. Indeed, in the $\widehat{G}$ affine quiver theory with $N_{i}=N_{0} d_{i}$, giving $\phi$ and $Q_{i j}$ dimensions via the superconformal chiral primary relation $\Delta=3 R / 2$ with the conserved anomaly free R-charges (11.4),

$$
\Delta\left(\phi_{i}\right)=\frac{3}{k+1}, \quad \Delta\left(Q_{i j}\right)=\frac{3 k}{2(k+1)},
$$

implies that all exact beta functions vanish. The exact beta functions which vanish are (4.7) for the gauge couplings, (4.8) for the superpotential couplings $\lambda_{i j}$, and (11.2) for the couplings $s_{i}$ in $W_{i}\left(\phi_{i}\right)=\frac{s_{i}}{k+1} \operatorname{Tr} \phi_{i}^{k+1}$.

The conditions (11.5) are necessary and sufficient for having an $\mathcal{N}=1$ superconformal theory. For the moment we will assume that, for general $k$ in (11.1), the equations (11.5) have solutions for some values of the couplings, $s_{i}^{*}, g_{i}^{*}$, and $\lambda_{i j}^{*}$, and discuss the resulting $\mathcal{N}=1$ superconformal theories.

Note that, as in [49], the conditions (11.5) for a superconformal theory are fewer in number than the number of adjustable coupling constants $g_{i}, s_{i}$, and $\lambda_{i j}$. This is because 
the $\beta\left(g_{i}\right)$ given by (4.7) are proportional to the $\beta\left(\lambda_{i j}\right)$ for the affine quiver theories with $N_{i}=N_{0} d_{i}$, for any $N_{0}$. Since the $\beta\left(g_{i}\right)$ vanishing equations are redundant, for all $i=0 \ldots r$ running over all nodes of the affine quiver diagram, there are $r+1$ fewer conditions on the fixed point couplings than there are unknowns. Thus, if there is any solution $s_{i}^{*}, g_{i}^{*}$, and $\lambda_{i j}^{*}$, there will be a $r+1$ dimensional moduli space of such solutions, with $r+1$ exactly marginal operators. Including the theta angles, this is a $r+1$ complex dimensional moduli space, with $r+1$ complex moduli.

Thus, for all $k$, the superconformal field theories are expected to have a $r+1$ complex dimensional moduli space of couplings, similar to the $r+1$ dimensional moduli space of couplings of the $\mathcal{N}=2$ affine quiver theories. The modulus corresponding to the diagonal $U(N)$ is related to the IIB string coupling, with its $S L(2, Z)$ S-duality group. The others are expected to have an S-duality group given by the $G$ Weyl group.

Assuming that these fixed points exist, we can determine their $a$ and $c$ central charges, as in [50], in terms of the 't Hooft anomalies of the $U(1)_{R}$ symmetry in the supercurrent multiplet:

$$
a-c=\frac{1}{16} \operatorname{Tr} U(1)_{R}, \quad 5 a-3 c=\frac{9}{16} \operatorname{Tr} U(1)_{R}^{3} .
$$

The 't Hooft anomalies $\operatorname{Tr} U(1)_{R}$ and $\operatorname{Tr} U(1)_{R}^{3}$ get contributions only from the massless chiral fermions. Consider first the $\mathcal{N}=2 \widehat{G}$ affine quiver theories with $N_{i}=N_{0} d_{i}$. The appropriate $U(1)_{R}$ symmetry to use in (11.6) is that under which $R(\phi)=R(Q)=2 / 3$, and $(11.6)$ then gives

$$
a_{\mathcal{N}=2}=c_{\mathcal{N}=2}=a_{\text {free }}=\frac{9 N^{2}\left|\Gamma_{G}\right|}{32}\left(1+\left(-\frac{1}{3}\right)^{3}+2\left(-\frac{1}{3}\right)^{3}\right)=\frac{N_{0}^{2}\left|\Gamma_{G}\right|}{4}
$$

where the terms are from the gauginos, and fermionic components of $\phi$ and $Q_{i j}$ respectively and we used $\sum_{i} d_{i}^{2}=\frac{1}{2} \sum_{i j}\left|s_{i j}\right| d_{i} d_{j}=\left|\Gamma_{G}\right|$. The central charge is independent of the moduli $\tau_{i}$, which is why it had to agree with the free field values, as in (11.7).

Now consider the $\mathcal{N}=1$ theories with deforming superpotential as in (11.1), for general $k$. The appropriate $U(1)_{R}$ symmetry assignments are as in (11.3) and then (11.6) gives for the central charges

$$
\begin{aligned}
a_{\mathcal{N}=1}(k)=c_{\mathcal{N}=1}(k) & =\frac{9 N^{2}\left|\Gamma_{G}\right|}{32}\left(1+\left(\frac{2}{k+1}-1\right)^{3}+2\left(\frac{k}{k+1}-1\right)^{3}\right) \\
& =\frac{27 k^{2} N_{0}^{2}\left|\Gamma_{G}\right|}{16(k+1)^{3}} .
\end{aligned}
$$


This expression has a maximum value at $k=2$, where $a_{\mathcal{N}=1}(k=2)=a_{\mathcal{N}=2}=a_{\text {free }}$, coinciding with (11.8). For all other $k \neq 2$, we have $a_{\mathcal{N}=1}(k)<a_{\text {free. }}$. According to the conjectured $a$ theorem, $a$ decreases along RG flows to the IR. Since $a_{N=1} \leq a_{N=2}$, the conjectured $a$ theorem is compatible with a RG flow from the $\mathcal{N}=2$ superconformal fixed points in the UV to our claimed $\mathcal{N}=1 \mathrm{RG}$ fixed points in the IR. The fact that $a_{\mathcal{N}=1}<a_{\text {free }}$ for $k \neq 2$ gives evidence that these theories indeed flow to an interacting RG fixed point rather than a free field theory.

Note that $a_{\mathcal{N}=1}(k=2)=a_{\text {free }}$, so the existence of a $\mathcal{N}=1$ RG fixed point for $k=2$ could potentially violate a strong form of the $a$ theorem, where $a$ must decrease along flows unless the theories are related by an exactly marginal operator. The $\mathcal{N}=1$ theory with $k=2$ looks like a deformation of the $\mathcal{N}=2$ theory by the operators $\sim \operatorname{Tr} \phi_{i}^{3}$, which are not exactly marginal (they're marginally irrelevant near the $\mathcal{N}=2$ line of fixed points). It could be, though, that the $\mathcal{N}=1$ theories exist, but just can't be obtained by starting from the $\mathcal{N}=2$ fixed points. Or it could be that the strong form of the $a$ theorem does not hold here (even in 2d, it's known that a non-compact target space can invalidate the $c$-theorem [51] ).

The AdS/CFT correspondence supports the existence of the $\mathcal{N}=1$ superconformal theories for general $k$ in (11.1) and general $\widehat{G}$ quiver diagram with $N_{i}=N_{0} d_{i}$. The AdS/CFT correspondence for the $k=1$ theories was discussed in [22], and this can be generalized for all $k$. Write the metric near the singularity of $X_{6}(k, \widehat{G})$ as $d s_{6}^{2}=d r^{2}+r^{2} d s_{5}^{2}$, with $d s_{5}^{2}$ the metric of the base $M_{5}(k, \widehat{G})$, with $M_{5}$ a 5 d Einstein manifold. The AdS/CFT correspondence here would be between IIB string theory on $M_{k, \widehat{G}}$ and our general $(k, \widehat{G})$ superconformal theories.

We can also check the above field theory exact results (11.8) for $a$ and $c$ with our associated geometry by using the AdS/CFT relation of [52]: regarding the 3 -fold $X$ associated with the $\mathcal{N}=1$ affine quiver theories with $W_{i} \sim \operatorname{Tr} \phi_{i}^{k+1}$ as a cone over base $M_{5}$, the prediction is that

$$
a_{\mathcal{N}=1}=c_{\mathcal{N}=1}=\frac{\pi^{3} N_{0}^{2}}{4 \operatorname{Vol}\left(M_{5}\right)}
$$

normalized so that the $\mathcal{N}=4$ case is $M_{5}=S^{5}$ of unit radius.

Though the Calabi-Yau metric is not known, the relevant volume appearing in (11.9) can still be found. The case $k=1$ was recently analyzed in [53], and the discussion there can be immediately generalized to our general $k$ case. Consider a general Calabi-Yau $n$ fold $X$ with a conical singularity which can be written as $F\left(z_{0}, \ldots z_{n}\right)=0$, up to resolving 
terms, with $F\left(\lambda^{w_{0}} z_{0}, \ldots, \lambda^{w_{n}} z_{n}\right)=\lambda^{d} F\left(z_{0}, \ldots z_{n}\right)$ homogeneous. Then the $2 n-1$ real dimensional base $B$ of the cone has volume [53]

$$
\operatorname{Vol}(B)=\frac{2 d}{(n-1) ! \prod w_{i}}\left(\frac{\pi\left(\sum w_{i}-d\right)}{n}\right)^{n} .
$$

We can apply this formula to our deformed geometry, where $n=3$, with $z_{0} \rightarrow t$ and $z_{i} \rightarrow X_{i}$. So $d=k C_{2}(G)$ and the weights are

$\begin{array}{cccccc}\Gamma_{G} & w_{0} & w_{1} & w_{2} & w_{3} & d \\ A_{r} & 1 & \frac{1}{2}(r+1) k & \frac{1}{2}(r+1) k & k & (r+1) k \\ D_{r} & 1 & (r-1) k & (r-2) k & 2 k & 2(r-1) k \\ E_{6} & 1 & 6 k & 4 k & 3 k & 12 k \\ E_{7} & 1 & 9 k & 6 k & 4 k & 18 k \\ E_{8} & 1 & 15 k & 10 k & 6 k & 30 k\end{array}$

with $w_{1}=\frac{1}{2} C_{2}(G) k$ and $d=k C_{2}(G)$. Plugging these into (11.10), note that in all cases $\sum w_{i}-d=k+1$ and $\prod_{i} w_{i}=k^{3} C_{2}(G)\left|\Gamma_{G}\right| / 4$, so we get

$$
\operatorname{Vol}\left(M_{5}\right)=\frac{4 \pi^{3}(k+1)^{3}}{27 k^{2}\left|\Gamma_{G}\right|} .
$$

Using (11.12) in (11.9) gives a result which agrees perfectly with the field theory result (11.8) for all $G$ and $k$.

Though we have given some arguments supporting the existence of the general $\mathcal{N}=1$ RG fixed points discussed above, we should also mention the possibility that there might be no solution of the conditions (11.3) needed for a superconformal theory when $k \geq 2$. One can see a possible problem for $k=2$ by considering the perturbative expressions for the anomalous dimensions $\$$. Considering a single $U\left(N_{c}\right)$ factor in the quiver theory, which has $N_{f}=2 N_{c}$, the exact beta functions are (up to positive proportionality factors)

$$
\beta(s) \sim \gamma(\phi), \quad-\beta(g) \sim \beta(\lambda) \sim \gamma(\phi)+2 \gamma(Q) .
$$

Thus a RG fixed point requires $\gamma(\Phi)=\gamma(Q)=0$. For $s=0$ there is an IR attractive fixed line of arbitrary $\lambda=g$, which is the $\mathcal{N}=2$ superconformal fixed line. Deforming by small $s$ one finds $\gamma(Q) \sim \lambda^{2}-g^{2}$ and $\gamma(\phi) \sim \lambda^{2}-g^{2}+c s^{2}$ with $c$ a positive constant, suggesting that there is no solution of $\gamma(Q)=\gamma(\phi)=0$ for $s \neq 0$. The signs of the beta functions are such that $s$ and $\lambda$ are driven to smaller values, whereas $g$ is driven to larger

4 We thank I. Klebanov and J. Polchinski for pointing this out to us, and for related discussions. 
values. Eventually the theory could perhaps hit a RG fixed point outside the region of validity for the above approximations for $\gamma(Q)$ and $\gamma(\Phi)$. The $\mathcal{N}=1$ fixed points could also exist outside the basin of attraction of the deformed $\mathcal{N}=2$ theories. Also, we should anticipate the need to include the dynamics of the coupled gauge group factors in order to see the possible $\mathcal{N}=1 \mathrm{RG}$ fixed points - turning off the dynamics of the neighboring gauge groups can lead to a free theory.

One can also consider this issue by using the duality of 44,45] between $\mathcal{N}=1$ supersymmetric $S U\left(N_{c}\right)$ gauge theory, with $N_{f}$ fundamentals and adjoint $\phi$ and superpotential

$$
W_{\text {elec }}=\frac{s}{k+1} \operatorname{Tr} \phi^{k+1}+\lambda \bar{Q} \phi Q
$$

and the $S U\left(N_{f}-N_{c}\right)$ magnetic dual, with similar superpotential. For $N_{f}=2 N_{c}$, rather than flowing to a non-trivial fixed point, both the electric and magnetic theories could be IR free (which is possible here since both have the same spectrum). But the non-trivial duality map suggests that, especially upon gauging the flavor symmetries, there could in fact be a non-trivial fixed point for general $k$.

\section{Quiver Theories from Local CY 3-folds}

So far we have discussed type IIB probe theories involving 3-brane and 5-branes wrapping cycles in a local 3-fold which has compact 2-cycles, but no compact 4-cycles. Furthermore we have talked about transitions in geometry which is interpreted as the large $N$ dual of the corresponding gauge theory. On the other hand CY 3-folds are known also to have transitions where some 4-cycles and 2-cycles shrink to zero size and where there is a transition involving emergence of non-vanishing 3-cycles. A notable example along this line is the del Pezzo transitions, where a del Pezzo surface (complex dimension two) shrinks inside a threefold and some three cycles emerge on the other side. More generally, the 4cycles can have several components, and each of the components can shrink. It is natural to ask what the corresponding quiver gauge theories are in such cases where 4-cycles shrink with wrapped D3,D5 and D7 branes, and what is the large $N$ dual interpretation of the corresponding geometric transition involving the emergence of 3-cycles.

There are two well known classes of this type. One is obtained by considering $C^{3} / \Gamma$ where $\Gamma$ is a subgroup of $S U(3)$, and the other is when we consider the local threefold to be realized torically. These two sets have overlap when $\Gamma$ is abelian. One could also consider more general examples which are neither toric, nor orbifolds. Our construction of quiver 
theories applies to all these cases. However we will mostly specialize to the case of local threefolds which can be realized torically. The advantage of restricting to the toric case is that the mirror symmetry action is simpler to study (though it can also be carried out in the other cases). These correspond to geometries that are realized in the linear sigma model approach [54] by considering the Higgs branch of a $G=U(1)^{k}$ gauge system with $k+3$ matter fields $x_{i}$ with charges $Q_{i}^{j}$, with $j=1, \ldots, k$ satisfying

$$
\sum_{i} Q_{i}^{j}=0
$$

One also has (complexified) FI terms given by $t^{j}=r^{j}+2 \pi i \theta^{j}$. The geometry is given by considering

$$
M=\left\{\sum_{i} Q_{i}^{j}\left|x_{i}\right|^{2}=r^{j}\right\} / / G
$$

which is a local Calabi-Yau 3-fold.

Before we consider branes in such geometries we have to find a good description of these backgrounds. The description just given is adequate when $r^{j}>>0$. But our regime of interest is not in large sizes for the 2- and 4-cycles, but rather when the sizes vanish. In this limit quantum corrections, due to worldsheet instantons will be quite important. To bypass having to compute these corrections, we will consider the mirror type IIA description, which already has the IIB quantum corrections summed up into a classical description. Following [55] we introduce dual periodic variables $Y_{i}$ satisfying $\operatorname{Re} Y_{i}=\left|x_{i}\right|^{2}$. Define $y_{i}=e^{-Y_{i}}$, so that $y_{i}$ are single valued complex numbers. Then the dual theory is given by a LG theory with superpotential $\sum_{i} y_{i}$ with the constraints

$$
\prod_{i} y_{i}^{Q_{i}^{j}}=e^{-t^{j}}
$$

Note that using these relations we can get rid of all the $y_{i}$ 's except for three. Let us call them $y_{1}, y_{2}, y_{3}$. By the homogeneity condition we can write

$$
\sum y_{i}=y_{3} f\left(y_{1} / y_{3}, y_{2} / y_{3}, t^{j}\right)
$$

As has been shown in [19] this is equivalent to (see also the earlier work [56]) considering the 3 -fold given by (redefining $y_{1} / y_{3} \rightarrow y_{1}$ and $y_{2} / y_{3} \rightarrow y_{2}$ )

$$
u v=f\left(y_{1}, y_{2}, t^{j}\right)
$$


where $u, v \in \mathbf{C}$ and $y_{1}, y_{2} \in \mathbf{C}^{*}$. In order to study Lagrangian D6 branes (wrapping 3-cycles and filling the spacetime), which is the mirror of D3,D5 and D7 branes, it is convenient to define

$$
W=f\left(y_{1}, y_{2}, t^{j}\right)
$$

The 3-fold geometry is thus given by

$$
W=f\left(y_{1}, y_{2}, t^{j}\right) \quad u v=W
$$

The advantage of writing it in this way is that, as shown in [19], one can split the study of the Lagrangian branes as a fibered geometry involving 2-cycles in the $y_{1}, y_{2}$ space, times the circle in the $u, v$ plane. The space of compact 2-cycles over $W=f\left(y_{1}, y_{2}\right)$ with boundary over a point $W=W_{0}$ is the subject of singularity theory [40 and is in 1-1 correspondence with the critical points of $W$, i.e. with solutions to

$$
\partial_{y_{1}} W=\partial_{y_{2}} W=0
$$

Let us label the critical points by $p_{\alpha}$ with $\alpha=1, \ldots, r$, and define $W_{\alpha}=W\left(p_{\alpha}\right)$. The geometry of these cycles with boundary over $W=W_{0}$ can be viewed as a disk where the boundary of the disk is identified with a circle over $W=W_{0}$ and vanishing at the critical points, along a path over the $W$ plane connecting $W_{\alpha}$ to $W_{0}$. Let us call the corresponding cycle $\Delta_{\alpha}$. To obtain 3 -cycles we take the product of this disc with the circle corresponding to circle action on the $u v$ plane. However this does not yield a closed 3-cycle. To remedy this we note that if we take $W=W_{0}$ then for a generic point on the path over the $W$ plane we have an $S^{1} \times S^{1}$ fiber, which as we approach $W_{\alpha}$ one $S^{1}$ shrinks and as we approach $W=0$ the other $S^{1}$ shrinks. This is therefore an $S^{3}$. Let us continue calling the corresponding class of $S^{3}$ by $\Delta_{\alpha}$. We thus have $r$ distinct classes of three cycles. This construction was already made in this context in [19]. See figure 5. 


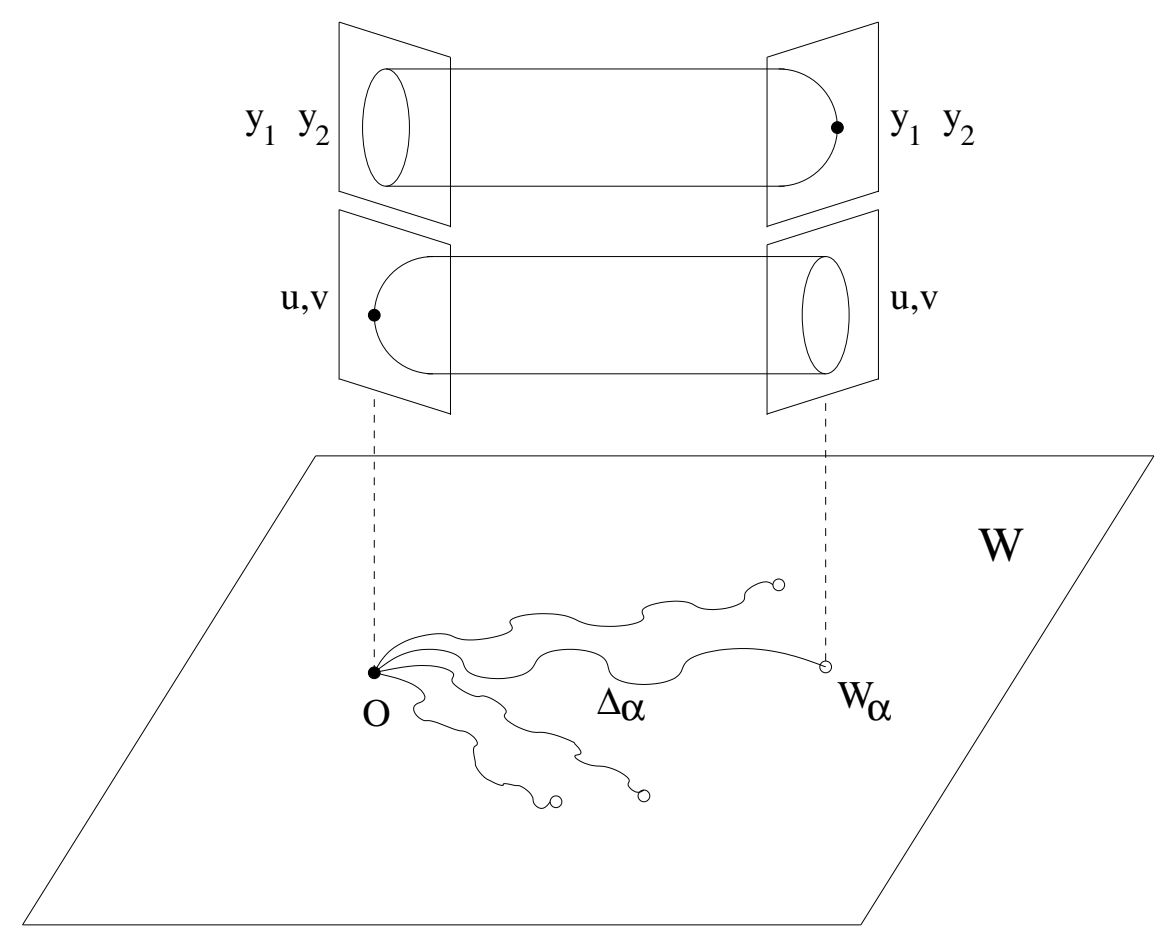

Figure 5:Identification of 3-cycles with $S^{3}$ topology for each path connecting one of the critical points of $W$ with $W=0$.

In order to map these cycles to the D3,D5 and D7 brane charges we need to know how the $r$ classes $\Delta_{\alpha}$ map to the allowed charges in the IIB description. The basic idea of how to determine this was shown in [19] for the case of toric del Pezzos $\left(P^{2}\right.$ blown up with up to three points, and $\left.P^{1} \times P^{1}\right)$. Namely, each $\Delta_{\alpha}$, in the large volume limit, can be identified as the mirror of a brane configuration on the compact 4-cycles in the CY geometry. In fact, for the case of del Pezzos, these theories have been recently studied in [57] using the ideas in [19], and our discussion here for the construction of the quiver theory is similar to it. The case studied in [19] was mainly for the Fano case, but the same ideas apply to compact cycles inside CY 3-folds.

Let us denote the corresponding brane with $V_{\alpha}$ which can also be viewed as a bundle (or more precisely a sheaf) on the type IIB side supported on the compact cycles of the CY. Using ideas from mirror symmetry the Chern character for $V_{\alpha}$ leads to the brane charges for $\Delta_{\alpha}$. The class of D3 brane is generally simple to determine: Note that, as in 58 ] this should map to a $T^{3}$ in the mirror which consists of the phases of $u, y_{1}, y_{2}$. Note that a representative of this class can be chosen to have large $\left|y_{1}\right|,\left|y_{2}\right|$ and so its intersection with all the $\Delta_{\alpha}$ which are at finite values of $y_{i}$ is zero. Let

$$
\left[T^{3}\right]=\sum_{\alpha=1}^{r} d_{\alpha} \Delta_{\alpha}
$$


Thus $N_{0}$ D3 branes get mapped to $N_{0} d_{\alpha}$ D6 branes wrapped over $\Delta_{\alpha}$ as $\alpha=1, \ldots, r$. We will choose $d_{\alpha} \geq 0$, if necessary by reversing the orientation of the corresponding $\Delta_{\alpha}$. Thus the theory of $N_{0} D 3$ branes gives rise to a gauge theory with gauge group

$$
\prod_{\alpha} U\left(N_{0} d_{\alpha}\right)
$$

This is an $\mathcal{N}=1$ supersymmetric gauge theory. There is also chiral matter which is in one to one correspondence with the intersection of the cycles. In particular there are

$$
n_{\alpha \beta}=\Delta_{\alpha} \circ \Delta_{\beta}
$$

net chiral fields transforming in the bifundamental of $U\left(N_{0} d_{\alpha}\right) \times U\left(N_{0} d_{\beta}\right)$ (where here we are including the extra circle coming from $u v=$ const in the definition of $\Delta_{\alpha}$ ). There is another interpretation of these intersection numbers, in terms of the associated $\mathcal{N}=2 \mathrm{LG}$ theory in $2 \mathrm{~d}$, defined by the superpotential $W\left(y_{1}, y_{2}\right)$, namely

$$
n_{\alpha \beta}=\operatorname{Tr}_{\alpha \beta}(-1)^{F} F
$$

where the right hand side is the index defined in [59] for the associated LG theory which counts the net number of BPS kinks between the vacua $\alpha$ and $\beta$. Note that the intersection number is topological and the actual number of matter fields may be more, but there is nothing preventing non-chiral pairs to pair up by mass deformations and we assume to be in this generic situation. Note that $n_{\alpha \beta}=-n_{\beta \alpha}$ and the sign of $n_{\alpha \beta}$ correlates with the chirality of the matter. In terms of the type IIB description the classes $\Delta_{\alpha}$ get mapped to bundles $V_{\alpha}$ (or sheaves). These should correspond to rigid bundlest (as the topology of the mirror is $S^{3}$ and has no $b_{1}$ which counts the deformation parameter of the brane [58]). These rigid bundles are sometimes referred to as spherical bundles. More precisely, a spherical bundle (or sheaf) $\mathcal{F}$ on a Calabi-Yau threefold is one that satisfies $\operatorname{Ext}^{i}(\mathcal{F}, \mathcal{F})=(\mathbf{C}, 0,0, \mathbf{C})$ for $i=(0,1,2,3)$, corresponding to the cohomology of $S^{3}$, where $\mathrm{Ext}^{i}$ is the analog of $H^{i}$ in the sheaf theoretic context. An example is $\mathcal{O}_{\mathbf{P}^{1}}$ where the Calabi-Yau is $\mathcal{O}(-1) \oplus \mathcal{O}(-1)$. Another class of examples is any line bundle on a component of a shrinking 4-cycle in a Calabi-Yau, as we will check below.

5 These sheaves are not bundles on the Calabi-Yau, but rather are bundles on the local 4-cycles. We will refer to them as bundles for ease of discussion. 
If $V_{\alpha}$ and $V_{\beta}$ are bundles on the same component of a shrinking 4-cycle $S$, then the intersection numbers $n_{\alpha \beta}$ are given in this type IIB setup, as will be discussed below, by

$$
n_{\alpha \beta}=\chi\left(V_{\alpha}, V_{\beta}\right)-\chi\left(V_{\beta}, V_{\alpha}\right)
$$

where $\chi\left(V_{\alpha}, V_{\beta}\right)$ denotes the Euler class of the $\bar{\partial}$ complex mapping sections of $V_{\alpha} \rightarrow V_{\beta}$, where here we are ignoring the embedding of the 4-cycle inside the Calabi-Yau. This relation has also been noted in [60,57. This is the IIB dual of the intersection number of the cycles. By Riemann-Roch, this simplifies to

$$
n_{\alpha \beta}=c_{1}(S) \cdot\left(c_{1}\left(V_{\beta}\right)-c_{1}\left(V_{\alpha}\right)\right),
$$

where $S$ denotes the union of complex surfaces inside the CY which can shrink and on which the bundles $V_{\alpha}, V_{\beta}$ are supported. Generically there is a choice of bundles, called an exceptional collection, for which for each pair $\alpha, \beta$ at most either $\chi\left(V_{\alpha}, V_{\beta}\right)$ or $\chi\left(V_{\beta}, V_{\alpha}\right)$ is non-zero, and moreover all the $H^{i}$ are zero except for $H^{0}\left(V_{\alpha}^{*} \otimes V_{\beta}\right)$, corresponding to holomorphic sections of maps from $V_{\alpha} \rightarrow V_{\beta}$ This implies that for each pair of branes $\alpha, \beta$ we choose an ordering so that $n_{\alpha \beta} \geq 0$. Then the $n_{\alpha \beta}$ matter fields $Q_{\alpha \beta}^{i}$, with $i=1, \ldots, n_{\alpha \beta}$ matter fields are in 1-1 correspondence with $n_{\alpha \beta}$ holomorphic maps

$$
f_{\alpha \beta}^{i}: \quad V_{\alpha} \rightarrow V_{\beta}
$$

Let's now specialize this discussion to our local case. The actual cohomologies whose index yields the intersection numbers $n_{\alpha \beta}$ needs to be worked out. In this calculation we need to clearly distinguish between bundles on complex surfaces and sheaves on the CalabiYau, so we introduce temporary notation in the next two paragraphs for clarification. If $V_{\alpha}$ is a line bundle on a component of the shrinking 4-cycle, we now denote by $\widetilde{V}_{\alpha}$ the corresponding sheaf on the local Calabi-Yau. It will be helpful first to recall the result of a computation in [61], which says that

$$
\operatorname{Ext}^{i}\left(\widetilde{V}_{\alpha}, \widetilde{V}_{\alpha}\right)=\operatorname{Ext}^{i}\left(V_{\alpha}, V_{\alpha}\right) \oplus \operatorname{Ext}^{3-i}\left(V_{\alpha}, V_{\alpha}\right)^{*} .
$$

Since $V_{\alpha}$ is a line bundle, we get $\operatorname{Ext}^{i}\left(V_{\alpha}, V_{\alpha}\right)=H^{i}(\mathcal{O})$ which is $\mathbf{C}$ for $i=0$ and is 0 otherwise. This shows that $\widetilde{V}_{\alpha}$ is spherical, as claimed.

A straightforward extension of this calculation based on Lemma 3.16 of [61] shows that

$$
\operatorname{Ext}^{i}\left(\widetilde{V}_{\alpha}, \widetilde{V}_{\beta}\right)=H^{i}\left(V_{\alpha}^{*} \otimes V_{\beta}\right) \oplus H^{3-i}\left(V_{\beta}^{*} \otimes V_{\alpha}\right)^{*}
$$


This immediately implies (12.1) if we use $n_{\alpha \beta}=\chi\left(\widetilde{V}_{\alpha}, \widetilde{V}_{\beta}\right)=\sum_{i=0}^{3}(-1)^{i} \operatorname{dimExt}^{i}\left(\widetilde{V}_{\alpha}, \widetilde{V}_{\beta}\right)$. But we can see more. A collection of bundles $\left\{V_{\alpha}\right\}$ defines an exceptional collection if for any pair of distinct bundles $V_{\alpha}, V_{\beta}$, then only one of $V_{\alpha}^{*} \otimes V_{\beta}$ or $V_{\beta}^{*} \otimes V_{\alpha}$ has cohomology, and that one has cohomology in only one degree.

Note that this theory is anomaly free, because the net number of chiral matter for the gauge group $U\left(N_{0} d_{\alpha}\right)$ is given by

$$
\sum_{\beta} N_{0} d_{\beta} n_{\alpha \beta}=N_{0} \sum_{\beta} d_{\beta}\left(\Delta_{\alpha} \circ \Delta_{\beta}\right)=N_{0}\left(\Delta_{\alpha} \circ\left[T^{3}\right]\right)=0 .
$$

In addition the theory will have some superpotential involving the chiral matter fields. 6 Consider a term in the superpotential, which by gauge invariance is of the form

$$
W=a_{i_{1} \ldots i_{k}} \operatorname{Tr} Q_{\alpha_{1} \alpha_{2}}^{i_{1}} \ldots Q_{\alpha_{l} \alpha_{l+1}}^{i_{l}} \ldots Q_{\alpha_{k} \alpha_{1}}^{i_{k}}
$$

with $i_{l}=1, \ldots, n_{\alpha_{l} \alpha_{l+1}}$ with $k+1 \equiv 1$. It is natural to try to relate $a_{i_{1} \ldots i_{k}}$ to properties of the ring structure of the corresponding holomorphic maps in the type IIB description. Note that this is natural because there is a natural product structure on the holomorphic maps between bundles, namely the composition of maps. Consider

$$
f_{\alpha_{1} \alpha_{2}}^{i_{1}} \ldots f_{\alpha_{k} \alpha_{1}}^{i_{k}}
$$

which will be a holomorphic map from $V_{\alpha_{1}}$ to itself and by assumption the only non-trivial class here is $H^{0}$ which is the identity map. So this composition will be a multiple of identity and so we have

$$
f_{\alpha_{1} \alpha_{2}}^{i_{1}} \ldots f_{\alpha_{k} \alpha_{1}}^{i_{k}}=a_{i_{1} \ldots i_{k}} \mathbf{1}_{\alpha_{1}}
$$

which we propose as defining the couplings of the superpotential. For $k=3$ this is obvious as this can be viewed as the overlap of the corresponding wavefunctions, and this is exact in the type IIB side. We believe this result also holds for arbitrary $k$, and we have verified

6 These are in 1-1 correspondence with the potential ' $1 / 4$ BPS instantons' in an $\mathcal{N}=22 \mathrm{~d}$ LG theory with superpotential $W\left(y_{1}, y_{2}\right)$ (for a study of these see 62 ). To see this note that disk instanton configurations can be organized in terms of a cyclic ordering of vacua at infinity as we go along a big circle, $\alpha_{1}, \ldots, \alpha_{k}$ and a choice of a $1 / 2$ BPS kink between any such pair, which is given by a chiral field $Q_{\alpha_{l} \alpha_{l+1}}^{i}$, where $i$ can be a number from 1 to $n_{\alpha_{l} \alpha_{l+1}}$ (assuming it is positive). Thus each such instanton configuration is in one to one correspondence with an allowed superpotential term. 
this in a number of examples. In some of the examples we consider we find that the $E x t$ group appears at degree 1 . In such a case, the same formula applies where we use the $E x t^{1}$ to deform the relevant bundles and compute the product of sections for the deformed bundles. This will be discussed in the context of $\mathbf{P}^{1} \times \mathbf{P}^{1}$ below.

In addition the above theories have FI terms. As we change the $t^{j}$, not only do we change the coupling constants, as the volume of 3-cycles change, but we also generate FI terms, as is well known [63]. Note that even though there are $r$ gauge groups, the number of $t^{j}$ 's is given by the number of 2-cycles in the original type IIB geometry, which is less than $r$ by the number of 4-cycles $n_{4}$ plus zero cycle (which equals 1 ). Taking into account the possible variation of the string coupling constant $\tau$ which is the coupling constant that the D3 brane sees, we see that the coupling constants and the FI terms of the $r$ gauge groups satisfy $n_{4}$ constraints.

So far we have only discussed the quiver theory for pure D3 branes. We can also have more general brane configurations involving D5 and D7 branes, which leads to a more general quiver theory where everything is as for the D3 branes, except that the rank of the gauge groups are now arbitrary (instead of being a multiple of $d_{\alpha}$ ). This is exactly as in the A-D-E quiver theories we studied before. However there is one difference between this case and the $\mathcal{N}=1 \mathrm{~A}-\mathrm{D}-\mathrm{E}$ quiver theories studied earlier: Whereas the previous theories were non-chiral and anomaly free, the present theories are chiral and in some cases are not anomaly free. This can be easily understood from the type IIB string theory setup as well. Consider for example a D3 branes, which corresponds to a point in the transverse 6-dimensional space. If the 6-cycle were compact then the theory would have problem supporting the D3 brane as the flux would have nowhere to go. Namely we consider the 6-cycle and we delete where the 3-brane is located then the topological fact that the $S^{5}$ surrounding the 3 -brane is topologically trivial in the compact 6-cycle implies that it cannot support any 5-form flux. However the same argument applies also to 5-branes and 7-branes, which wrap 2 and 4 dimensional cycles in the transverse space. Namely if there is a compact dual cycle for any of them then we can use that cycle to undue any $S^{3}$ or $S^{1}$ surrounding the corresponding brane. Thus the only allowed total brane charges consistent with flux conservation are in 1-1 correspondence with cycles in $H_{*}$ which intersect no compact cycle. In particular all the 4-cycles are ruled out, because they intersect the 2-cycles inside them. Of the 2-cycles we can choose classes which do not intersect the 4-cycles (i.e. are not electric-magnetic duals). In particular there are $n_{2}-n_{4}$ 
such choices where $n_{2}$ denotes the number of inequivalent 2-cycles and $n_{4}$ the number of 4-cycles.

One has to be careful that here we are discussing the total charge of the branes. There is no problem with having D3,D5 and D7 branes wrap arbitrary cycles as long as the total class does not intersect the compact cycles.

We can also understand this result from the condition of having an anomaly free quiver theory. Suppose we wrap $N_{\alpha}$ branes over the $\Delta_{\alpha}$ cycle. Then the condition for having no anomalies is that

$$
\sum_{\beta} N_{\beta} n_{\alpha \beta}=0
$$

for each $\alpha$, which means that the vector $N$ is in the null subspace of the matrix $n$. Recall that the matrix $n_{\alpha \beta}$ is, by a change of basis, an anti-symmetrized intersection form of the 0-,2- and 4-cycles. Thus indeed this is exactly the condition that the cycle represented by $N$ has no intersection with any other compact cycle, as was anticipated. Thus in general there are

$$
r^{\prime}=1+n_{2}-n_{4}=r-2 n_{4}
$$

inequivalent integers controlling the rank of the $r$ gauge groups where $r=1+n_{2}+n_{4}$.

Note that the ideas presented above for construction of the quiver theory could also have been carried out for the case of the general 4-cycle shrinking inside the CY 3-folds, and not just the toric case. This can be done directly in the type IIB setup, without appealing to mirror symmetry. Namely we would have to choose a collection of exceptional spherical bundles, as many as necessary to get all the allowed brane charges, namely $r$. Of course there would be $2 n_{4}$ constraint for the multiplicities of these bundles. Then the quiver diagram can be constructed by computing the Ext group between pairs of such bundles, and Yukawa couplings can be computed as discussed above, by the composition of the holomorphic maps between bundles. However the reason we have discussed the type IIA mirror in the toric case is that, just as in [4], the relevant worldsheet quantum corrections become classical and leads to a simple understanding of Seiberg-like dualities.

\subsection{Two Examples: $\mathbf{P}^{2}$ and $\mathbf{P}^{1} \times \mathbf{P}^{1}$}

Let us consider two simple examples to illustrate these ideas: $\mathbf{P}^{2}$ and $\mathbf{P}^{1} \times \mathbf{P}^{1}$ inside a CY 3-fold. These cases were studied in detail in [19], and here we use the discussion above to write down the corresponding quiver theory. 
Consider first the $\mathbf{P}^{2}$ case. Then the threefold is given by

$$
u v=W \quad W=y_{1}+y_{2}+\frac{e^{-t}}{y_{1} y_{2}}+1
$$

This arises from a linear sigma model with a single $U(1)$ gauge group with 4 fields with charges $(-3,1,1,1)$. Here $t$ denotes the complexified Kahler class of $\mathbf{P}^{2}$. The three critical points of $W\left(y_{1}, y_{2}\right)$ are given by $y_{i}=\omega e^{-t / 3}$ where $\omega$ is a third root of unity, which yields the critical values in the $W$ plane on three points on a tiny circle of radius $\left|e^{-t / 3}\right|$ near $W=1$. See Figure 6 . The three $\Delta$ 's are depicted in Figure 6 and connect each of these three critical values to $W=0$. As discussed in [19] these are in 1-1 correspondence in the type IIB setup with branes wrapping $\mathbf{P}^{2}$ and representing bundles $\mathcal{O}(-1), \mathcal{O}(0), \mathcal{O}(1)$. If we label these $\alpha$ 's by $1,2,3$ respectively, we have

$$
n_{12}=n_{23}=3 \quad n_{13}=6
$$

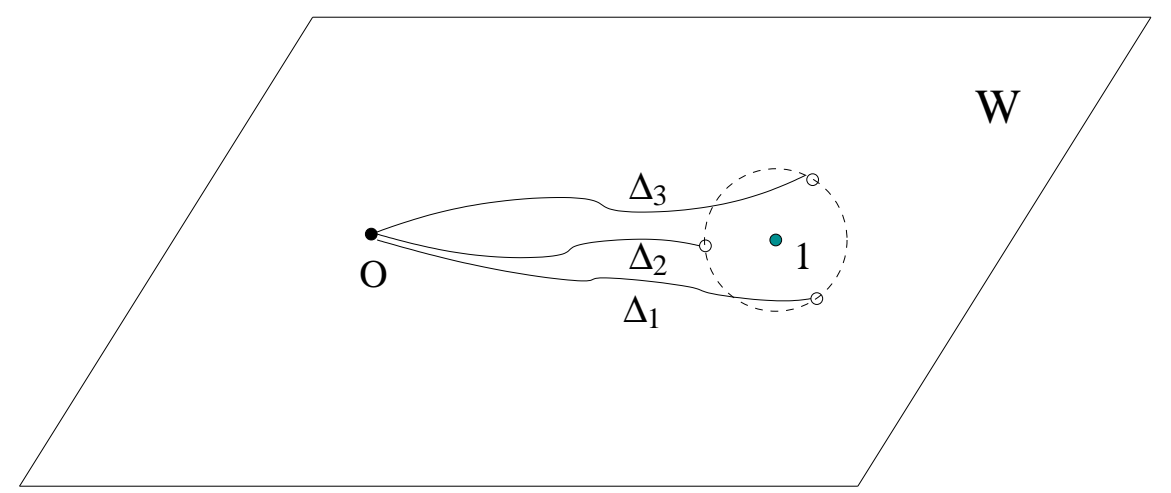

Figure 6:Projection of 3-cycles on the $W$ plane for the mirror of a three-fold with a $\mathbf{P}^{2}$.

Note that these are in one to one correspondence with the holomorphic maps from bundles, namely

$$
\begin{aligned}
& A_{i}:(12) \quad \mathcal{O}(-1) \rightarrow \mathcal{O}(0) \leftrightarrow H^{0}(\mathcal{O}(1))=3 \\
& B_{i}:(23) \quad \mathcal{O}(0) \rightarrow \mathcal{O}(1) \leftrightarrow H^{0}(\mathcal{O}(1))=3 \\
& C_{i j}:(13) \quad \mathcal{O}(-1) \rightarrow \mathcal{O}(1) \leftrightarrow H^{0}(\mathcal{O}(2))=6
\end{aligned}
$$

where $i, j$ take values $1,2,3$ and $C_{i j}$ is a symmetric tensor. There are no other maps or cohomologies between any pairs of these bundles. Note that the sections $A_{i}$ and $B_{j}$ can be identified with degree one homogeneous function of $\left(z_{1}, z_{2}, z_{3}\right)$ so we can choose a basis where

$$
A_{i} \leftrightarrow z_{i} \quad B_{j} \leftrightarrow z_{j}
$$




$$
C_{i j} \leftrightarrow z_{i} z_{j}
$$

The class corresponding to pure D3 brane charge is given, as follows from [19], by

$$
[\mathcal{O}(-1)]-2[\mathcal{O}(0)]+[\mathcal{O}(1)]=\left[\Delta_{1}\right]-2\left[2 \Delta_{2}\right]+\left[\Delta_{3}\right]
$$

which means that to get positive $d_{\alpha}$ we need to reorient

$$
\Delta_{2} \rightarrow \Delta_{2}^{\prime}=-\Delta_{2}
$$

in which case we obtain $d_{1}=1, d_{2}=2, d_{3}=1$, and moreover

$$
n_{21}=n_{32}=3 \quad n_{13}=6
$$

Note that the signs of $n_{12}, n_{23}$ have changed due to a change in sign in the orientation of $\Delta_{2}$. We can now write the corresponding superpotential using the identification with sections of the bundle and the corresponding multiplication, which gives

$$
W=\sum_{i, j=1}^{3} A_{i} B_{j} C_{i j}
$$

Note that in this case $r^{\prime}=r-2=1$ so there is only one inequivalent choice for rank, which simply corresponds to putting $N_{0}$ D3 branes, giving the gauge group $U\left(N_{0}\right) \times U\left(N_{0}\right) \times$ $U\left(2 N_{0}\right)$. This reflects the statement that for $P^{2}$ the $P^{1}$ cycle in $P^{2}$ intersects $P^{2}$ and no $D 5$ or $D 7$ branes are allowed. It can also be readily checked that the matrix $n_{\alpha \beta}$ has only one null eigenvector.

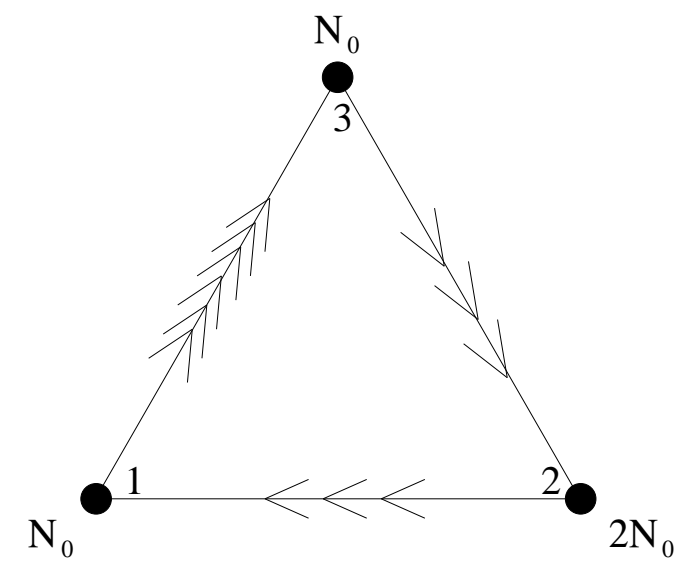

Figure 7:Quiver diagram corresponding to the field theory realized on the world volume of D3 branes. 
For the $\mathbf{P}^{1} \times \mathbf{P}^{1}$ we get the mirror description

$$
u v=W \quad W=y_{1}+\frac{e^{-t_{1}}}{y_{1}}+y_{2}+\frac{e^{-t_{2}}}{y_{2}}+1
$$

where $t_{1}, t_{2}$ denote the complexified volumes of the two $\mathbf{P}^{1}$ 's. The linear sigma model corresponding to this has two $U(1)$ charges and 5 fields with charges $(1,1,0,0,-2)$ and $(0,0,1,1,-2)$. The $\mathbf{P}^{1}$ 's are realized by the fields 1,2 and fields 3,4 . Note that the difference of these two $U(1)$ 's shows that in this geometry there is a $\mathbf{P}^{1}$ with area $t_{1}-t_{2}$ in the class being the difference of the two $\mathbf{P}^{1}$ 's whose normal bundle is $\mathcal{O}(-1)+\mathcal{O}(-1) \rightarrow \mathbf{P}^{1}$ since this yields the charges $(1,1,-1,-1,0)$. We will use this fact later.

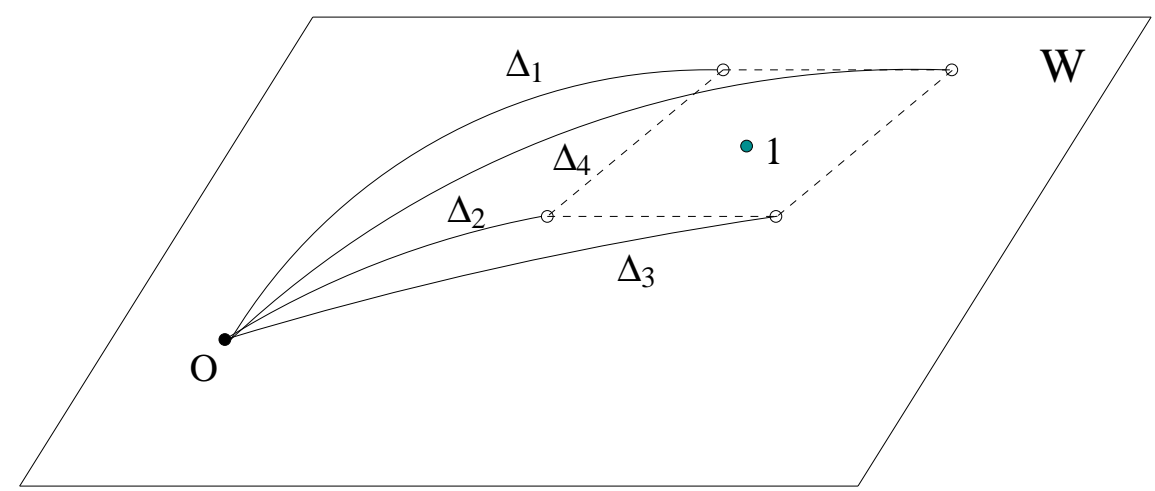

Figure 8:Projection of 3-cycles on the $W$ plane for the mirror of a three-fold with a $\mathbf{P}^{1} \times \mathbf{P}^{1}$.

There are four critical points of $W$ and thus there are four $\Delta$ 's as shown in Fig. 8. As follows from [19] the $\Delta$ 's are in one to one correspondence with $\mathcal{O}(-1)_{1} \otimes \mathcal{O}(-1)_{2}, \mathcal{O}(0)_{1} \otimes$ $\mathcal{O}(-1)_{2}, \mathcal{O}(0)_{1} \otimes \mathcal{O}(0)_{2}, \mathcal{O}(-1)_{1} \otimes \mathcal{O}(0)_{2}$, which gives

$$
n_{12}=n_{23}=2 \quad n_{14}=n_{43}=2 \quad n_{13}=4
$$

moreover they are in 1-1 correspondence with monomials

$$
n_{12}, n_{43}: z_{1}^{i} \quad n_{23}, n_{14}: z_{2}^{i} \quad n_{13}: z_{1}^{i} z_{2}^{j}
$$

where $i, j$ are 1,2 and the subscript of $z$ denotes the choice of the $\mathbf{P}^{1}$, and there are no other maps or cohomologies. The class corresponding to pure D3 brane is given by

$$
\left[\Delta_{1}\right]-\left[\Delta_{2}\right]+\left[\Delta_{3}\right]-\left[\Delta_{4}\right]
$$


which means that if we wish to get positive $d_{\alpha}$ we switch the orientation of $\left[\Delta_{2}\right] \rightarrow$ $-\left[\Delta_{2}\right],\left[\Delta_{4}\right] \rightarrow-\left[\Delta_{4}\right]$. Note that this yields

$$
n_{21}=n_{32}=2 \quad n_{41}=n_{34}=2 \quad n_{13}=4
$$

The superpotential can be easily deduced from the multiplication of sections and we obtain

$$
W=\sum_{i, j=1}^{2} A_{i} B_{j} D_{i j}+A_{i}^{\prime} B_{i}^{\prime} D_{j i}
$$

where $D_{i j}$ corresponds to the 4 diagonal chiral fields (no symmetry condition on the indices imposed) and $A_{i}, B_{j}$ are matter fields on the edges 32,21 and $A_{i}^{\prime}, B_{j}^{\prime}$ are the matter fields on the edges 34,41 .

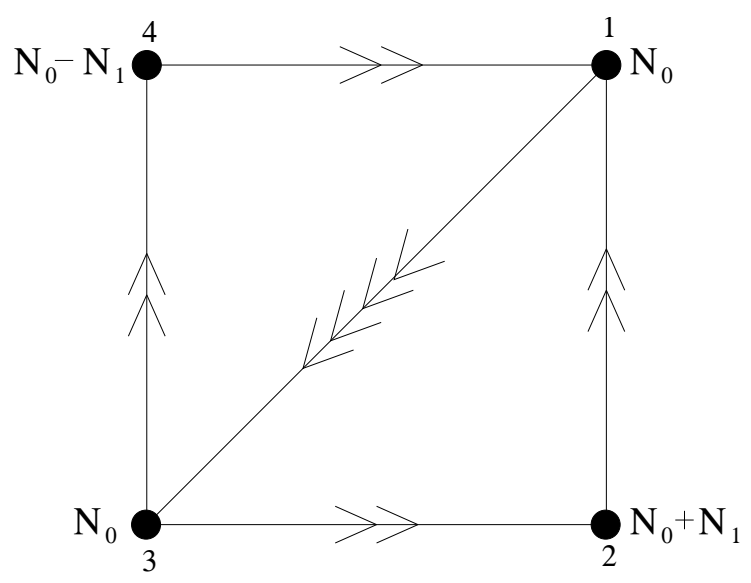

Figure 9:Quiver diagram for the field theory corresponding to $\mathbf{P}^{1} \times \mathbf{P}^{1}$.

In this case we expect to have the possibility of adding one more brane charge because $r^{\prime}=r-2=4-2=2$. This corresponds to the fivebrane wrapping the 2-cycle class given by the difference of the two $\mathbf{P}^{1}$, as that will not have any intersection with the 4-cycle given by $\mathbf{P}^{1} \times \mathbf{P}^{1}$. This class is given by the class represented by $\left[\Delta_{4}\right]-\left[\Delta_{2}\right]$. Suppose we put $N_{1}$ branes in that class, and $N_{0} 3$-brane. In this case the gauge theory will be $U\left(N_{0}\right) \times U\left(N_{0}+N_{1}\right) \times U\left(N_{0}\right) \times U\left(N_{0}-N_{1}\right)$ (ordered according to node number). One can check that this is the only anomaly free choice of ranks allowed in this example, as expected. 


\subsection{Geometric Transitions and Seiberg-like Dualities for Chiral $\mathcal{N}=1$ Quiver Theories}

In the type IIB setup the dualities arise by flops in Kahler cones, as first studied in [3]. However in cases we are considering the type IIA mirror is more convenient as the quantum worldsheet corrections are absent, as noted above. In this context the dualities in field theories arise by considering varying the parameters of the bulk and using conservation of brane charge to deduce a dual description as in [4], which we follow here: We consider a quiver theory arising from the toric theories discussed above which in the mirror type IIA description is given by $N_{\alpha}$ D6 branes wrapping $\Delta_{\alpha}$ and filling the spacetime. This leads to the gauge group $\prod_{\alpha} U\left(N_{\alpha}\right)$ with $\Delta_{\alpha} \circ \Delta_{\beta}=n_{\alpha \beta}$ chiral fields transforming in $\left(N_{\alpha}, \bar{N}_{\beta}\right)$ or $\left(\bar{N}_{\alpha}, N_{\beta}\right)$ depending on the sign of $n_{\alpha \beta}$. The theory would also have some superpotential and some FI terms. The coupling constant $g^{2}$ of the $U\left(N_{\alpha}\right)$ group is inversely proportional to the volume of the $\Delta_{\alpha}$ cycle. The dualities typically arise when the coupling goes to infinity, which occurs when the cycle $\Delta_{\alpha}$ goes to zero size, and then it becomes negative, which means that another cycle has emerged and we wish to find the new gauge description which would be a dual quiver theory. Now there are many inequivalent topological configurations the theory may emerge in: There are in fact $r-2$ possibilities depending on which wedge, in the $W$ plane, the new $\Delta_{\alpha}^{\prime}$ cycle emerges, as shown in Fig. 10.
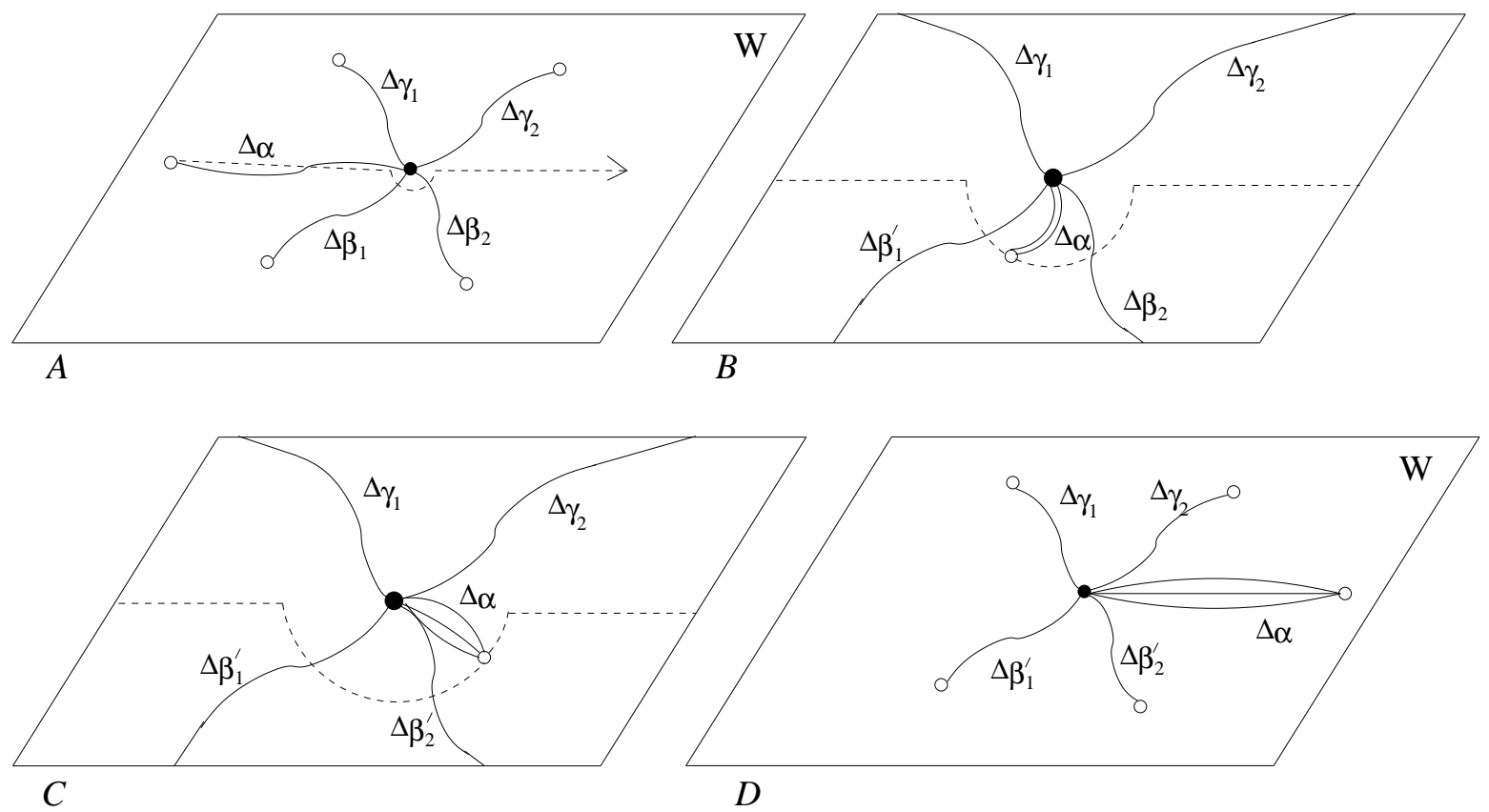

Figure 10:(A) Trajectory of the critical point of $W$ corresponding to cycle $\Delta_{\alpha}$. The point actually crosses the origin, but a small detour is taken in order to compute the changes in the charges. (B) Close-up of the first crossing. The class of $\Delta_{\beta_{1}}$ changes and brane creation takes place. (C) 
Close-up of the second crossing, $\Delta_{\beta_{2}}$ changes and brane creation takes place again. (D) Final configuration.

The brane charge conservation gives the rank of the dual theory. In fact, as shown in [19] in this case we create new branes. Strictly speaking the case studied in [19] did not have the extra circle coming from $u v=W$, in the D-brane worldvolume, but this does not affect the arguments of [19].

The easiest way to find the brane charge is to avoid passing through the singular point, by going around it. As will be evident from the result, the resulting brane charge conservation yields the same result independent of which way we go around the $W=0$ point. The transition line of the quiver divides the $W$ plane and thus the set of gauge groups to two parts. Let us denote the ones on one side by $\beta_{i}$ and the other by $\gamma_{j}$. As we move the $\Delta_{\alpha}$ passing through the $\Delta_{\beta}$ 's, it creates new $\Delta_{\alpha}$ branes given by

$$
-N_{\beta_{i}} n_{\beta_{i} \alpha}
$$

Also after passing through the singularity it is natural to reorient the brane (for preserving the same supercharge this is necessary). Thus the total number of $\Delta_{\alpha}$ branes are given by

$$
N_{\alpha}^{\prime}=\sum_{i} N_{\beta_{i}} n_{\beta_{i} \alpha}-N_{\alpha}
$$

Note that the rank of the other gauge groups do not change. However, the quiver diagram changes because the intersection of cycles has changed. This can be found using Picard Lefschetz theory and one finds the following: The intersections of the cycles with $\alpha$ has not changed except for the overall sign having to do with the reorientation of the $\alpha$ cycle. This means that the arrows ending or beginning on $\alpha$ will have the same degeneracy but opposite orientation. The intersection between the cycles among $\Delta_{\beta_{i}}$ or $\Delta_{\gamma_{j}}$ do not change, but the intersections between the pairs from these groups changes according to

$$
\left(\Delta_{\beta_{i}} \circ \Delta_{\gamma_{j}}\right)^{\prime}=n_{\beta_{i} \gamma_{j}}^{\prime}=n_{\beta_{i} \gamma_{j}} \pm n_{\beta_{i} \alpha} n_{\alpha \gamma_{j}} .
$$

This is the same as how the soliton numbers change in the corresponding $2 \mathrm{~d}$ LG theory [64], and the sign choice is correlated with the orientation of the cycles [64]. For our present case, we can write a simple expression which gives this sign choice, and which applies for any cycles $\sigma$ and $\rho$, whether they're $\beta_{i}$ 's or $\gamma_{j}$ 's:

$$
n_{\sigma \rho}^{\prime}=n_{\sigma \rho}+\frac{1}{2}\left(n_{\sigma \alpha}\left|n_{\alpha \rho}\right|+\left|n_{\sigma \alpha}\right| n_{\alpha \rho}\right)
$$


This expression is properly antisymmetric and captures all of the changes of the cycle intersections described above. In the original type IIB description where these cycles are mapped to a collection of exceptional bundles we get a new collection of exceptional bundles under the above operation. This is well known mathematically and is known as the mutation of exceptional bundle (for a review of this phenomenon in the physics literature see [65,19] ).

Let us consider a special case of the above transmutation which turns out to have a simple gauge theoretic interpretation. Let all the arrows from $\beta_{i}$ to $\alpha$ be incoming and all the ones from $\alpha$ to $\gamma_{j}$ be outgoing. In this case the change in the quiver diagram, corresponding to a different number of holomorphic maps between the mutated bundles as well as the change in the ring structure can be described as follows: Let $A_{\beta_{i} \alpha}^{l}$ denote the sections from $V_{\beta_{i}} \rightarrow V_{\alpha}$ with $l=1, \ldots, n_{\beta_{i} \alpha}$. Let $B_{\alpha \gamma_{j}}^{k}$ denote the sections from $V_{\alpha} \rightarrow V_{\gamma_{j}}$ with $k=1, \ldots, n_{\alpha \gamma_{j}}$ and $C_{\gamma_{j} \beta_{i}}^{p}$ denote the sections of $V_{\gamma_{j}} \rightarrow V_{\beta_{i}}$ with $p=1, \ldots, n_{\gamma_{j} \beta_{i}}$. Consider the ring for the sections which can be captured by the superpotential

$$
W=a_{l k p} A_{\beta_{i} \alpha}^{l} B_{\alpha \gamma_{j}}^{k} C_{\gamma_{j} \beta_{i}}^{p}+\ldots
$$

where ... all the other elements given by the ring structure. In the new quiver theory the $A$ 's and $B$ 's have disappeared and in the above superpotential they only appear in $A^{l} B^{k}$ combinations and will be replaced by a single new object $M^{l k}$ (the "meson field") in the $W$. Moreover instead of $A$ 's and $B$ 's we have new sections $A_{\alpha \beta_{i}}^{\prime l}, B_{\gamma_{j} \alpha}^{\prime k}$ with the same degenerancy but dual gauge quantum numbers and which appear in the above superpotential as $W \rightarrow W+B^{\prime k} A^{\prime l} M^{l k}$. This is of course nothing but the Seiberg duality on the factor represented by the node $\alpha$. Note that $W$ now has a quadratic piece given by

$$
a_{l k p} M_{\beta_{i} \gamma_{j}}^{l k} C_{\gamma_{j} \beta_{i}}^{p}
$$

which can be integrated out to lead to a net $n_{\beta_{i} \alpha} n_{\alpha \gamma_{j}}-n_{\gamma_{j} \beta_{i}}$ chiral fields in the bifundamental representation of $\left(\beta_{i} \gamma_{j}\right)$, in agreement with (12.2). The description of $W$ and the objects given above encode the holomorphic maps between transmuted bundles as well as the ring structure between them. Moreover in the field theory setup what we have done is Seiberg duality, as was proposed in the context of $\mathcal{N}=1$ chiral quiver theories in [21]. Here we see that Seiberg duality is simply a special case of what happens when a cycle shrinks and another evolves which is realized as mutation of bundles on the type IIB side, and the brane creation and the Picard Lefschetz monodromy in the type IIA side. For a special 
class of theories (branes in the del Pezzo background) and when $N_{f}=2 N_{c}$ for the node to be dualized, the connection between variation of Kahler moduli and Seiberg duality was noted in [20] [21]. Also for the same special cases the connection to Picard-Lefschetz monodromy was pointed out in [21]. 0

Here we have found a more general possibility of quiver duality corresponding to the brane movements where not all the branes corresponding to incoming and outgoing fields are on the same side on the $W$ plane. This is not necessarily a field theory duality as the dynamics may or may not relate these theories to undergo mutation in an arbitrary way. It would be interesting to see which of these classes of quiver dualities are realized in field theory. Of course, this could in principle also be settled by going to the large $N$ dual holographic description as we have seen in the context of A-D-E quiver theories in this paper. It would be interesting to see if one can also understand the case of quiver mutation when not all the $\Delta_{\alpha}$ 's and $\Delta_{\beta}$ 's correspond to chiral and anti-chiral matter respectively, from the field theory viewpoint. One idea along this line is that effectively one has given mass to some of the flavors and then one does a Seiberg-like duality on less flavors and then after duality one takes the mass to zero. This idea is worth further study.

\subsection{Examples of Seiberg Dualities as Mutations: $\mathbf{P}^{2}, \mathbf{P}^{1} \times \mathbf{P}^{1}$}

Let us see how this works in the context of the examples we discussed before. Consider the $\mathbf{P}^{2}$ case. Suppose we change $t$ so that $t \rightarrow 0$ and then becomes negative. Note that the orbifold limit where the theory is equivalent to $\mathbf{C}^{3} / Z_{3}$ corresponds to $t \rightarrow-\infty$.

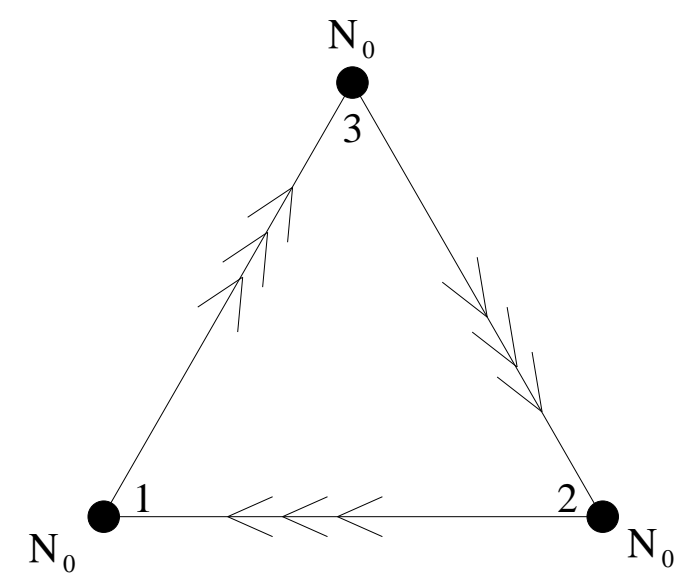

7 An important ingredient here, compared to those studied in [21] is the brane creation effect discussed in 19]. Taking this effect into account eliminates the claimed discrepancies between Seiberg duality and Picard-Lefschetz monodromy. 
Figure 11a:Quiver diagram after a mutation in the $\mathbf{P}_{2}$ example.

Since $\Delta_{2}$ has shrunk and emerged on the other side where it would have been with negative coupling, then we will end up dualizing the node 2, see Fig. 11b. According to our discussion above we obtain a mutated bundle in the type IIB configuration with quiver diagram given in Fig. 11a. Following Seiberg duality, which is equivalent to the quiver theory of the mutation, and replacing $A_{i} B_{j} \rightarrow M_{i j}$ and introducing the dual matter fields $A_{i}^{\prime}, B_{j}^{\prime}$ we obtain

$$
W=\sum_{i, j=1}^{3} A_{i} B_{j} C_{i j} \rightarrow W=M_{i j} C_{i j}+M_{i j} B_{j}^{\prime} A_{i}^{\prime}
$$

Noting that $C_{i j}$ is symmetric and $M_{i j}$ has no particular symmetry property, we see that the symmetric part of $M_{i j}$ pairs up with $C_{i j}$ and becomes massive, and we are left with the antisymmetric part of $M_{i j}$. Let us define $C_{k}^{\prime}=\epsilon_{i j k} M_{i j}$. Then the superpotential is

$$
W=\epsilon_{i j k} C_{k}^{\prime} B_{j}^{\prime} A_{i}^{\prime}
$$

which is the superpotential expected at the orbifold point, obtained using the methods of 66] in 67.

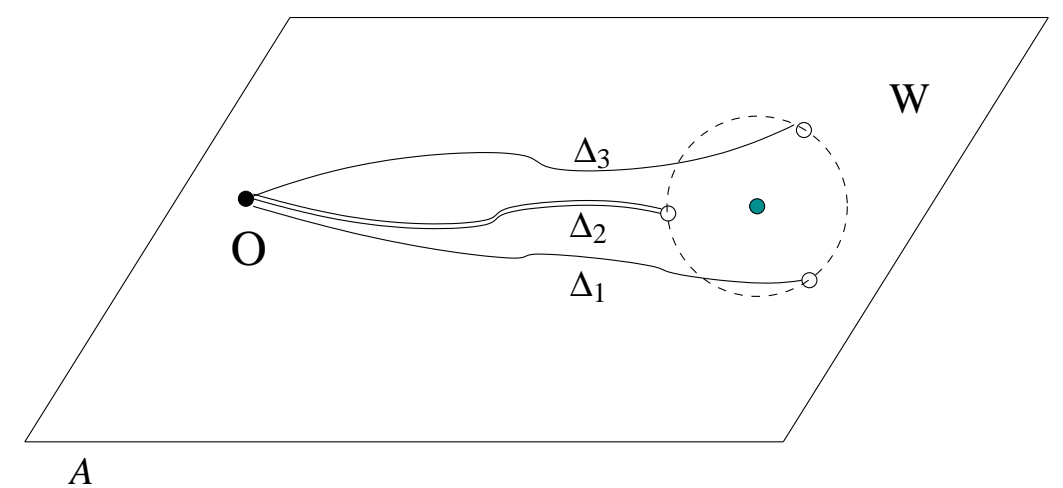

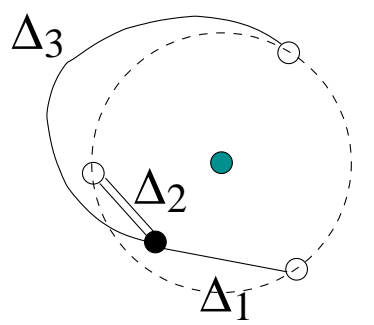

$B$

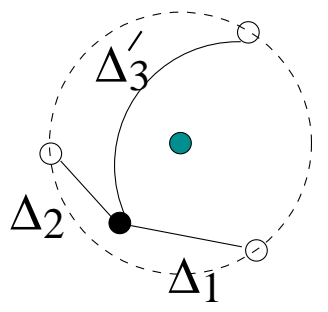

C

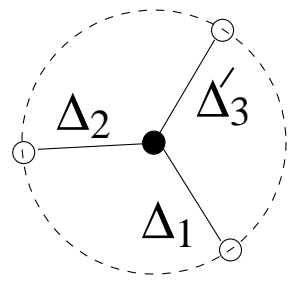

$D$

Figure 11b:Mutation in the $\mathbf{P}^{2}$ example. Notice that the projection of the $\Delta_{2}$ cycle actually becomes zero, but a small detour has been taken in order to compute the new charges. 
Let's now obtain the quiver of Figure 11a and the superpotential of (12.3) by geometry. Mutating $\mathcal{O}(1)$ to the left of $\mathcal{O}$, we get the rank 2 kernel of the multiplication map

$$
\mathcal{O} \otimes \operatorname{Hom}(\mathcal{O}, \mathcal{O}(1)) \rightarrow \mathcal{O}(1)
$$

which is identified with the bundle $\Omega^{1}(1)$, whose cohomologies are well known. The resulting bundles are $\mathcal{O}(-1), \Omega^{1}(1), \mathcal{O}$.

The intersection numbers are now $n_{i j}= \pm 3$. The maps are

$$
\begin{aligned}
& \operatorname{Hom}(\mathcal{O}(-1), \mathcal{O})=H^{0}(\mathcal{O}(1))=\mathbf{C}^{3} \\
& \operatorname{Hom}\left(\mathcal{O}(-1), \Omega^{1}(1)\right)=H^{0}\left(\Omega^{1}(2)\right)=\mathbf{C}^{3} \\
& \operatorname{Hom}\left(\Omega^{1}(1), \mathcal{O}\right)=H^{0}(T(-1))=\mathbf{C}^{3} .
\end{aligned}
$$

Note that $\Omega^{1}(2)$ is the kernel of the multiplication map

$$
\mathcal{O}(1) \otimes H^{0}(\mathcal{O}(1)) \rightarrow \mathcal{O}(2)
$$

so its sections are identified with the set of triples $\left\{\ell_{1}, \ell_{2}, \ell_{3}\right\}$ of linear forms such that $x_{1} \ell_{1}+x_{2} \ell_{2}+x_{3} \ell_{3}=0$, where $x_{i}$ are the homogeneous coordinates on $\mathbf{P}^{2}$. The combination $x_{1} \ell_{1}+x_{2} \ell_{2}+x_{3} \ell_{3}$ corresponds to the symmetric part of $M_{i j}=A_{i} B_{j}$, so the kernel is naturally identified with the antisymmetric part as expected. In other words, we have basis of sections identified with the triples

$$
\left\{\left(0, x_{3},-x_{2}\right),\left(-x_{3}, 0, x_{1}\right),\left(x_{2},-x_{1}, 0\right)\right\}
$$

which are obtained antisymmetrically. The identification of $\left(\ell_{1}, \ell_{2}, \ell_{3}\right)$ with a section of $\Omega^{1}(2)$ is by

$$
\left(\ell_{1}, \ell_{2}, \ell_{3}\right) \mapsto \ell_{1} d x_{1}+\ell_{2} d x_{2}+\ell_{3} d x_{3}
$$

The identification of the $A_{i}^{\prime}$ with sections of $T(-1)$ is via

$$
\left(A_{1}^{\prime}, A_{2}^{\prime}, A_{3}^{\prime}\right) \mapsto A_{1}^{\prime} \frac{\partial}{\partial_{x_{1}}}+A_{2}^{\prime} \frac{\partial}{\partial_{x_{2}}}+A_{3}^{\prime} \frac{\partial}{\partial_{x_{3}}}
$$

So the composition of the maps $\mathcal{O}(-1) \rightarrow \Omega^{1}(1) \rightarrow \mathcal{O}$ is given by

$$
\left(A_{1}^{\prime} \frac{\partial}{\partial_{x_{1}}}+A_{2}^{\prime} \frac{\partial}{\partial_{x_{2}}}+A_{3}^{\prime} \frac{\partial}{\partial_{x_{3}}}\right) \cdot\left(\ell_{1} d x_{1}+\ell_{2} d x_{2}+\ell_{3} d x_{3}\right)=A_{1}^{\prime} \ell_{1}+A_{2}^{\prime} \ell_{2}+A_{3}^{\prime} \ell_{3}
$$


under the identification of maps $\mathcal{O}(-1) \rightarrow \mathcal{O}$ with sections of $\mathcal{O}(1)$. Direct calculation shows that $A_{i}^{\prime} C_{k}^{\prime}=\epsilon_{i j k} x_{j}$. Identifying $B_{j}^{\prime}$ with the dual of $x_{j}$ completes the calculation of the superpotential. Note that the result we have found for what the brane charges are and the bundles they correspond to in the orbifold limit is consistent with the result 68] where it was shown that the wrapped branes for the orbifold point just discussed can be identified with the exceptional collection $\mathcal{O}(-1), \Omega(1), \mathcal{O}(0)$, where $\Omega$ is the cotangent bundle of $\mathbf{P}^{2}$.

To this exceptional collection we can apply mutations, generating an infinite number of exceptional collections. In field theory, this corresponds to successively performing Seiberg duality at different nodes of the quiver gauge theory. By this procedure, we generate an infinite number of gauge theories that are Seiberg dual to each other. To interpret this infinite number of exceptional collections geometrically, we have to consider the Teichmüller space of this noncompact Calabi-Yau, rather than just its moduli space 69. The stringy moduli space of $\mathbf{C}^{3} / Z_{3}$ is given by a sphere with three punctures, the large volume limit, a conifold point and the orbifold point. Since the conifold point has a monodromy of infinite order, the Teichmüller space would be an infinite cover of the moduli space. In particular, there are infinite copies of the orbifold point. At each copy of the orbifold point we have a different set of wrapped branes, which are just the different exceptional collections, related by mutations.

It is possible to show that the number of chiral fields of the quiver theory, $x_{i}$, satisfy a Diophantine equation [64] (which was studied there in the context of solitons of $\mathbf{P}^{\mathbf{2}}$ sigma model):

$$
x_{1}^{2}+x_{2}^{2}+x_{3}^{2}=x_{1} x_{2} x_{3}
$$

Moreover the rank of the corresponding gauge groups $N_{i}$ on each of the three nodes of the quiver are given by

$$
N_{i}=N \frac{x_{i}}{3}
$$

where $N_{i}$ denotes the node facing the side with $x_{i}$ flavors. Furthermore, we would like to argue that the phenomenon of duality cascade is present also in this case. This is easiest to see if we start with the quiver theory of figure 11a, with gauge group $U(N)^{3}$. If we apply Seiberg duality to one of the nodes, we obtain the quiver theory of figure 7 , with gauge group $U(N) \times U(N) \times U(2 N)$. For each node of the original theory, we obtain a different copy of this theory. We can now perform Seiberg duality at each node of the new theories, and iterate the process. The structure that appears is presented in figure 12 


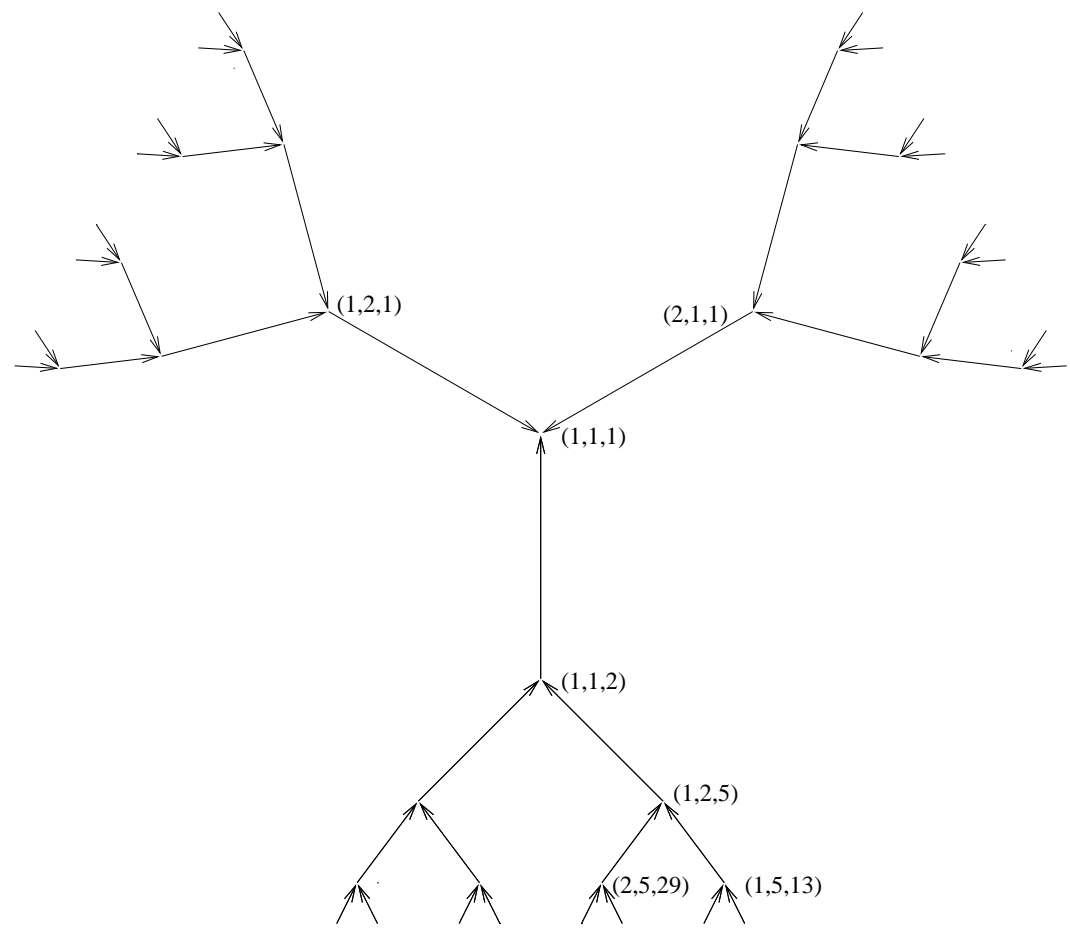

Figure 12:Structure of the cascade for the $\mathbf{P}^{2}$ case. Each node in the figure represents a gauge theory. The ranks $N_{i}$ for some of them are displayed (which can be rescaled by an overall factor of $N$ ). The arrows give the direction of the flow.

To prove that there is a duality cascade, we proceed as follows. Let's denote by $N_{1,2,3}$ the ranks of the three groups. First, it is easy to see that the number of flavors of the $U\left(N_{1}\right)$ gauge theory is given by $N_{2} x_{3}=N x_{2} x_{3} / 3$. The condition that $U\left(N_{1}\right)$ be asymptotically free is that $3 N_{1}>N x_{2} x_{3} / 3$ which is equivalent, using $N_{1}=N x_{1} / 3$ to $x_{1}>x_{2} x_{3} / 3$. It is not too difficult to show that for the largest rank, say $N_{1}$, this condition is satisfied. From this it follows that the group with largest rank is AF, whereas the other two are not. This implies that given any of these theories, as we flow to the IR, the coupling of the group with largest $N_{i}$ goes to infinity, and a Seiberg duality for that group is called for. After the duality, the situation repeats, the new group with largest $N_{i}^{\prime}$ is now the only AF theory, and repeatedly applying the same argument we see that as we flow to the IR, we encounter a cascade of dualities where the ranks of the gauge groups strictly decrease, until we reach the IR endpoint of the flow, the $U(N)^{3}$ theory. However, we should note that this duality cascade is different from the other ones we have talked about, in the sense that the number of 3-branes is the same throughout. Moreover, as we will discuss in the next section there is no geometric transition corresponding to blowing up 3-cycles in this case. 
For another example consider $\mathbf{P}^{1} \times \mathbf{P}^{1}$ discussed before. Let us consider the limit $t_{1}, t_{2} \rightarrow-\infty$ with $t_{1}-t_{2}=t$ being finite. This can also be viewed as the orbifold of $\mathcal{O}(-1) \oplus \mathcal{O}(-1) \rightarrow \mathbf{P}^{1}$ by the $Z_{2}$ acting on the fiber and fixing the $\mathbf{P}^{1}$. Then the $W$ plane geometry is given in Fig. 13 below, corresponding to $\Delta_{2}$ passing through zero. Doing Seiberg duality/mutation we obtain the quiver diagram given in Fig. 14. To obtain the superpotential we introduce the meson field $M_{i j}=A_{i} B_{j}$ and the dual matter fields $\widetilde{A}_{i}, \widetilde{B}_{j}$ with the superpotential

$$
W=\sum_{i j} M_{i j} D_{i j}+A_{i}^{\prime} B_{j}^{\prime} D_{j i}+M_{i j} \widetilde{B}_{j} \widetilde{A}_{i} .
$$

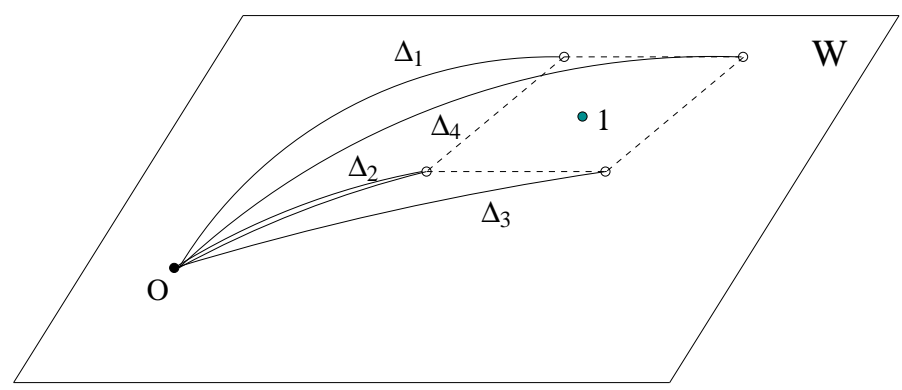

(A)
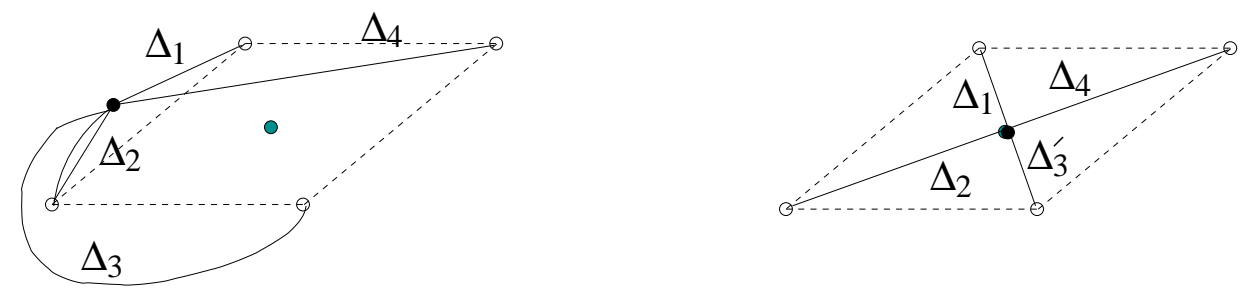

(B)

(C)

Figure 13:Mutation in the $\mathbf{P}^{1} \times \mathbf{P}^{1}$ example.

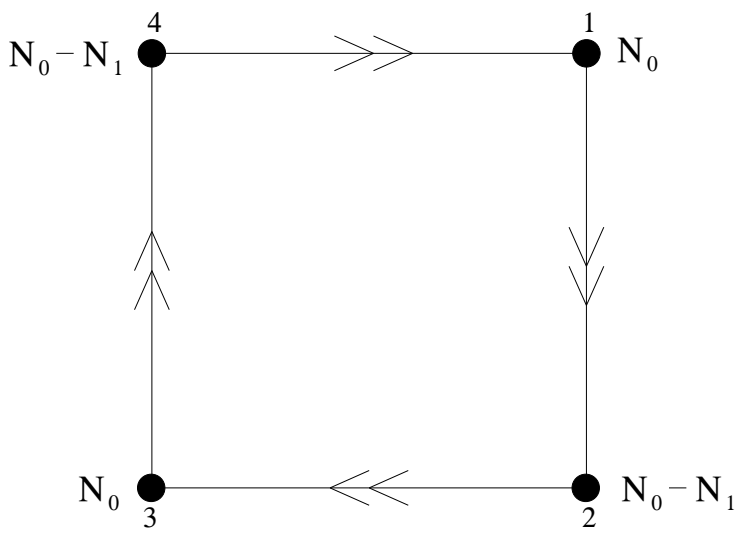


Figure 14:Quiver diagram for the $\mathbf{P}^{1} \times \mathbf{P}^{1}$ case after the mutation.

Integrating out $M_{i j}$ and $D_{i j}$ we obtain

$$
W=-A_{i}^{\prime} B_{j}^{\prime} \widetilde{B}_{i} \widetilde{A}_{j}
$$

which agrees with the results of [70] (which can be seen by redefining $A_{i}^{\prime}=\epsilon_{i k} F_{k}$ and $\left.B_{j}^{\prime}=\epsilon_{j r} G_{r}\right)$. Note that here the theory has $N_{0}$ three branes and $N_{1} 5$-branes wrapping the $\mathbf{P}^{1} / Z_{2}$. The superpotential for the 3-brane and 5-branes for $\mathbf{P}^{1}$ is the $\widehat{A}_{1}$ theory and the above theory is its $Z_{2}$ orbifold which is how [70] obtained $W$. This duality was already noted in [71] in the case with $N_{1}=0$, as the explanation of the two inequivalent quiver theories. Here we see in which regime of parameters each is naturally defined.

We can also see from the viewpoint of transmuted bundles how the quiver diagram and the Yukawa couplings arise. Performing the mutation, we replace $\mathcal{O}(0,0)$ with the kernel of $\mathcal{O}(0,-1) \otimes \operatorname{Hom}(\mathcal{O}(0,-1) \rightarrow \mathcal{O}(0,0))$. Since the Hom is two dimensional, the kernel is a line bundle. Since its Chern class is immediately calculated as $(0,-2)$, we see that the mutated bundle is $\mathcal{O}(0,-2)$. The collection of bundles is given by $\mathcal{O}(-1,-1), \mathcal{O}(0,-1), \mathcal{O}(0,-2), \mathcal{O}(-1,0)$. The nonzero Hom's and Ext's are

$$
\begin{aligned}
& \operatorname{Hom}(\mathcal{O}(-1,-1), \mathcal{O}(0,-1))=H^{0}(\mathcal{O}(0,1))=\mathbf{C}^{2} \\
& \operatorname{Hom}(\mathcal{O}(0,-1), \mathcal{O}(0,-2))=H^{0}(\mathcal{O}(0,1))=\mathbf{C}^{2} \\
& \operatorname{Ext}^{1}(\mathcal{O}(-1,0), \mathcal{O}(0,-2))=H^{0}(\mathcal{O}(1)) \otimes H^{1}(\mathcal{O}(-2))=\mathbf{C}^{2} \\
& \operatorname{Hom}(\mathcal{O}(-1,-1), \mathcal{O}(-1,0))=H^{0}(\mathcal{O}(0,1))=\mathbf{C}^{2}
\end{aligned}
$$

We reverse the signs of the bundles $\mathcal{O}(0,-2)$ and $\mathcal{O}(-1,0)$ (note that $c_{1}(\mathcal{O}(-1,-1))+$ $\left.c_{1}(\mathcal{O}(0,-1))-c_{1}(\mathcal{O}(0,-2))-c_{1}(\mathcal{O}(-1,0))=0\right)$. We check that the directions of the arrows also agree with those in Figure 14. Note that there was an extra arrow reversal for due to the $\operatorname{Ext}^{1}$ (i.e. the bundle $\operatorname{Hom}(\mathcal{O}(-1,0), \mathcal{O}(0,-2))$ has negative index). One can also obtain a duality cascade in this case as will be discussed in the next section.

Now we compute the Yukawa couplings. Classes $t \in \operatorname{Ext}^{1}(\mathcal{O}(-1,0), \mathcal{O}(0,-2))$ describe extensions $V_{t}$ of $\mathcal{O}(-1,0)$ by $\mathcal{O}(0,-2)$ fitting into an exact sequence

$$
0 \rightarrow \mathcal{O}(0,-2) \rightarrow V_{t} \rightarrow \mathcal{O}(-1,0) \rightarrow 0
$$

where the extension class $t$ is the obstruction to (12.5) defining $V_{t}$ as a direct sum. We compute Yukawa couplings between $V_{t}$ and the remaining two bundles $\mathcal{O}(-1,-1)$ and 
$\mathcal{O}(0,-1)$, then differentiate with respect to the components of $t$ to obtain the Yukawa couplings. As expected, the results will agree with the superpotential we found in (12.4).

We do the calculation by using a Cech cohomology representation. If $\left(x_{1}, x_{2}\right)$ and $\left(y_{1}, y_{2}\right)$ are coordinates on the respective $\mathbf{P}^{1}$ factors, for $i=1,2$ let $U_{i}$ be the open set on which $y_{i} \neq 0$. Then we represent classes in $\operatorname{Ext}^{1}(\mathcal{O}(-1,0), \mathcal{O}(0,-2)) \simeq H^{1}(\mathcal{O}(1,-2))$ on $U_{1} \cap U_{2}$ by the cocycle

$$
\rho_{t}=\frac{t_{1} x_{1}+t_{2} x_{2}}{y_{1} y_{2}}
$$

where $t=\left(t_{1}, t_{2}\right)$ is the Ext class. We define $V_{t}$ on each of the $U_{i}$ as the direct sum $\mathcal{O}(-1,0) \oplus \mathcal{O}(0,-2)$. To define $V_{t}$ globally, sections must be glued via the matrix

$$
\left(\begin{array}{cc}
1 & 0 \\
\rho_{t} & 1
\end{array}\right)
$$

We now compute the Hom's between $\mathcal{O}(0,-1), V_{t}, \mathcal{O}(-1,-1)$. We first have

$$
\operatorname{Hom}(\mathcal{O}(-1,-1), \mathcal{O}(0,-1))=H^{0}(\mathcal{O}(1,0))
$$

spanned by $x_{1}$ and $x_{2}$.

We also have $\operatorname{Hom}\left(\mathcal{O}(0,-1), V_{t}\right)=H^{0}\left(V_{t} \otimes \mathcal{O}(0,1)\right)$. This can be computed from the transition matrix (12.7), if the local sections are viewed as sections of the bundles $\mathcal{O}(-1,0) \otimes \mathcal{O}(0,1)=\mathcal{O}(-1,1)$ and $\mathcal{O}(0,-2) \otimes \mathcal{O}(0,1)=\mathcal{O}(0,-1)$. A basis for the holomorphic sections (expressed in the $U_{1}$ representation) may be taken to be

$$
V_{1}=\left(\begin{array}{c}
-\left(t_{1} x_{1}+t_{2} x_{2}\right) / y_{1} \\
y_{2}
\end{array}\right), V_{2}=\left(\begin{array}{c}
0 \\
y_{1}
\end{array}\right)
$$

Note that $\rho_{t} V_{i}$ is holomorphic in the $U_{2}$ variables so we do have global sections.

Finally, we have $\operatorname{Hom}\left(V_{t}, \mathcal{O}(-1,-1)\right)=H^{0}\left(V_{t}^{*} \otimes \mathcal{O}(-1,-1)\right)$. By dualizing (12.5) and tensoring with $\mathcal{O}(-1,-1)$, we get

$$
0 \rightarrow \mathcal{O}(0,-1) \rightarrow\left(V_{t}^{*} \otimes \mathcal{O}(-1,-1)\right) \rightarrow \mathcal{O}(-1,1) \rightarrow 0
$$

This is almost the same as (12.5); we just have to tensor (12.5) by $\mathcal{O}(0,1)$ and change the extension class from $t$ to $-t$ when we dualize. A basis for sections is then given by

$$
W_{1}=\left(\begin{array}{c}
\left(t_{1} x_{1}+t_{2} x_{2}\right) / y_{1} \\
y_{2}
\end{array}\right), W_{2}=\left(\begin{array}{c}
0 \\
y_{1}
\end{array}\right)
$$


Using (12.8) and (12.9), we compute the products of the Homs as

$$
V_{1} W_{2}=-t_{1} x_{1}-t_{2} x_{2}, V_{2} W_{1}=t_{1} x_{1}+t_{2} x_{2}, \quad V_{1} W_{1}=V_{2} W_{2}=0 .
$$

Now introducing variables $X_{1}, X_{2}$ for the sections $x_{1}, x_{2}$ of $\operatorname{Hom}(\mathcal{O}(-1,-1), \mathcal{O}(0,-1))$, we get

$$
V_{1} X_{i} W_{2}=-t_{i}, V_{2} X_{i} W_{1}=t_{i}, V_{i} X_{j} W_{i}=0 .
$$

Introducing $T_{i}$ for the Exts, we get be differentiation

$$
V_{1} X_{i} W_{2} T_{j}=-\delta_{i j}, V_{2} X_{i} W_{1} T_{j}=\delta_{i j}, V_{i} X_{j} W_{i} T_{k}=0 .
$$

This agrees with what we found earlier (12.4) by the identification $A_{i}^{\prime}=\epsilon_{i j} V_{j}, B_{j}^{\prime}=$ $X_{j}, \tilde{B}_{i}=W_{i}, \tilde{A}_{j}=T_{j}$.

\section{Large $N$ Dualities Involving Vanishing 4-Cycles}

It is well known that there are transitions in CY 3-folds where a 4-cycle shrinks and some three cycles grow. It was suggested in [7] that transitions of this kind may also realize some large $N$ duality in the context of wrapped branes. Here we will show that this is indeed the case. Start with the $A_{1}$ theory, i.e. with $N_{1}$ D5 branes wrapping $\mathbf{P}^{1}$. This gives the pure $\mathcal{N}=1$ theory with $G=U\left(N_{1}\right)$. It is dual to the conifold with $N_{1}$ units of $H$ flux through $S^{3}$. Now mod out both sides by $Z_{2}$ acting on the fiber direction of $\mathcal{O}(-1) \oplus \mathcal{O}(-1) \rightarrow \mathbf{P}^{1}$. Note that this corresponds to an $A_{1}$ singularity over $\mathbf{P}^{1}$ which if we blow up, gives $\mathbf{P}^{1} \times \mathbf{P}^{1}$. When they both shrink, it is an example of a shrunk 4-cycle. By following this through the transition this acts as $S^{3} / Z_{2}$ with no fixed points (the $S^{3}$ can be viewed as a sphere bundle over $\mathbf{P}^{1}$ which leads to this action). Thus we already have a concrete realization of these types of transitions. Note that on the quiver side the gauge theory this corresponds to is simply $U\left(N_{1}\right) \times U\left(N_{1}\right)$ with no matter fields (this is the special case of the quiver theory we studied with $N_{0}=0$ ), shown in Figure 14. Just as before $N_{1}$ controls the size of the $S^{3}$ and the gaugino condensate field is related to the modulus field of $S^{3}$. Note also that there are now two domain walls wrapping the $S^{3} / Z_{2}$ as discussed in [72,73], which is consistent with the fact that we have two inequivalent domain walls, one for each gauge factor.

There is also the duality cascade, just as we discussed in the context of the $\widehat{A}_{1}$ : Namely as the coupling gets weaker, as we go back in the UV (by taking $t$ to be a pure 
imaginary $B$ field and varying it) the geometry of the critical points on the $W$ plane change as shown in Fig. 15 and the gauge factor undergoes Seiberg dualities/mutations of the type we discussed and we end up in the UV with $U\left(k N_{0}\right)^{2} \times U\left((k+1) N_{0}\right)^{2}$ after $k$ cascades backward. Note that in this case the picture we had presented in section 10 for the mechanical analogy of the particle in the Coxeter box comes to life: The projection of the branes on the $\mathrm{W}$-plane are like the strings attached to the point inside the Coxeter box, which are related to the coupling of the gauge theories.
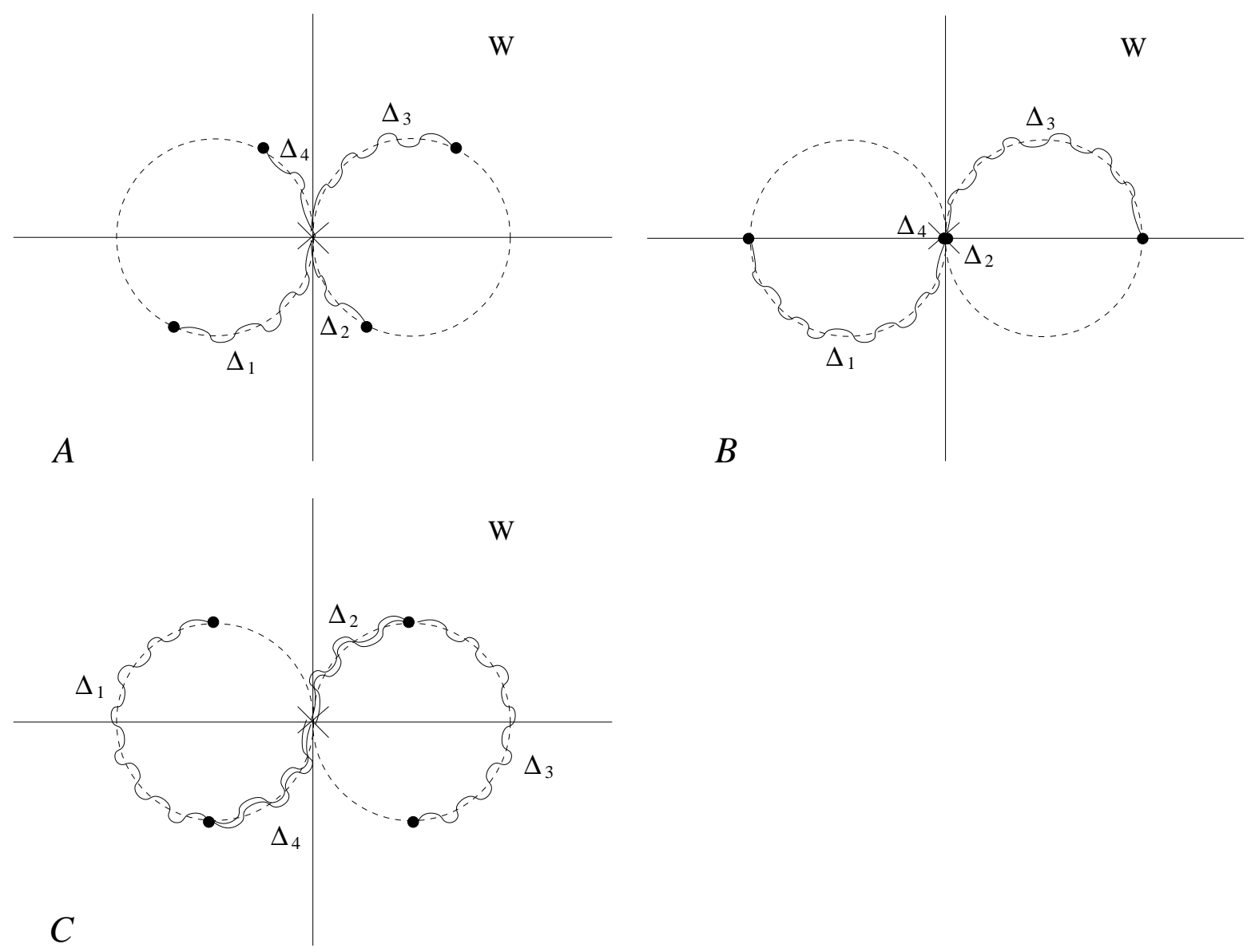

Figure 15:Realization of duality cascade in the context of $\mathbf{P}^{1} \times \mathbf{P}^{1}$ in the orbifold limit. The graph above corresponds to $t$ pure imaginary. The movement of the particle in the Coxeter Box, becomes physical in this context, and is identified with a semi-circle of either of the two circles above.

\subsection{Examples of Other 4-cycle/3-cycle transitions as large $N$ dualities}

The above example confirms the validity of the general idea. Here we will show other examples of this in the context of quiver theories associated with del Pezzos. We will first 
restrict ourselves to toric del Pezzos which are given, in addition to the $\mathbf{P}^{1} \times \mathbf{P}^{1}$ already studied, by $\mathbf{P}^{2}$ and its blowups with up to 3 points. Let us denote by $B_{k}$ the $P^{2}$ blown up at $k$ points. It is known that there are no transitions with $k=0,1$, and that for $B_{2}$ there is a transition controlled by one parameter and for $B_{3}$ there are two inequivalent transitions one involving 1 parameter and the other involving 2 parameters (these moduli spaces meet similar to how the $z$-axis meets the $x y$ plane). These results have been obtained in [74](see also 75 for generalities on smoothing other del Pezzo contractions). We will now see how to realize these transitions in the quiver theories arising from del Pezzos.

The corresponding quiver theories have already been constructed in [76,71] or they can be constructed with the methods discussed here (which are similar to those discussed in [57]). As already discussed we expect that $B_{k}$ will have a quiver theory with $r=k+3$ nodes with $k+1$ inequivalent integers labeling the number of 3 -brane and $k$ classes of 5 -branes. Note that the classes of 5 -brane charges is what affect the $H$ flux and thus can possibly control the moduli on 3 -cycles on the other side. So for $B_{k}$ we may expect $k$ deformations. Indeed it is known [75] that there are $k$ infinitesimal deformations for $B_{k}$ with $k=1,2,3$. But, as noted above some are obstructed.

The basic idea from physics is that the unobstructed ones should correspond to gaugino condensates in some pure $\mathcal{N}=1$ Yang-Mills theory, as was seen in all the cases encountered. Thus we look for possibilities of having such gauge theories in the corresponding quiver theory. We have already encountered exactly this structure in the context of $\mathbf{P}^{1} \times \mathbf{P}^{1}$, where in the general class of the quiver theory we could write one which has only pure $\mathcal{N}=1$ Yang-Mills. We got $U\left(N_{1}\right)^{2}$ with no matter, and the single factor $N_{1}$ controls the

size of the $S^{3}$. We now look for this in the other cases. For $\mathbf{P}^{2}$ this is not possible as already anticipated. So we move on to the other $B_{k}$ with $k=1,2,3$.

\section{$B_{1}$ and transition}

The quiver theory for this case is given in [77] and shown in Fig. 16 below. We can identify the nodes with the sheaves given by

$$
\begin{array}{ll}
1: & \mathcal{O}(H) \\
2: & \mathcal{O} \\
3: & \mathcal{O}(H-e) \\
4: & \mathcal{O}(e)
\end{array}
$$


where $H$ corresponds to the hyperplane class in $\mathbf{P}^{2}$ and $e$ is the class corresponding to the blow up. Also we have to reorient the nodes 3 and 4 . The arrow from 4 to 3 corresponds to an Ext as in the $\mathbf{P}^{1} \times \mathbf{P}^{1}$ example. It is easy to see that if we want $U(N)$ factors with no matter fields, compatible with allowed 5-brane charge labeled by $N_{1}$ this is not possible. Note that the allowed ranks are given by

$$
U_{1}\left(N_{0}\right) \times U_{2}\left(N_{0}-N_{1}\right) \times U_{3}\left(N_{0}+N_{1}\right) \times U_{4}\left(N_{0}-2 N_{1}\right)
$$

and to get a disconnected figure we need three of the nodes be zero without the fourth being zero and this is impossible. Thus this agrees with the geometric anticipation that there are no deformations in this case. Note also that the class given by the above $N_{0}$ and $N_{1}$ give

$$
N_{0} H+\left(N_{0}+N_{1}\right)(e-H)-\left(N_{0}-2 N_{1}\right)(e)=N_{1}(3 e-H)
$$

which is consistent with the fact that $c_{1} \cdot(3 e-H)=(3 H-e) \cdot(3 e-H)=0$ (using $\left.H^{2}=-e^{2}=1, H \cdot e=0\right)$.

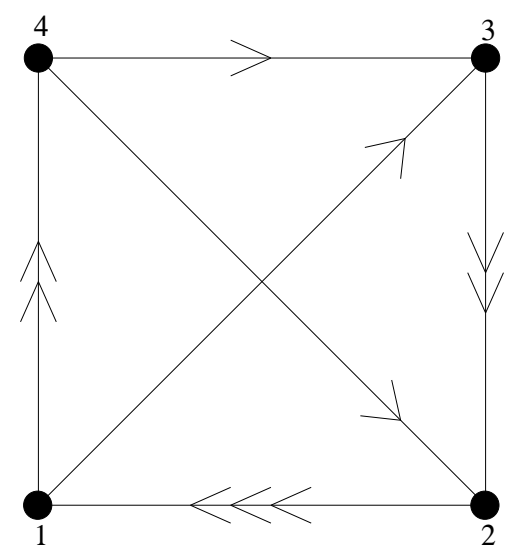

Figure 16:Quiver diagram for the field theory in the $B_{1}$ case.

\section{$B_{2}$ and transition}

The quiver for this case is given in [77]. There are some inequivalent choices (related by dualities). We have chosen one in Fig 17. To obtain pure $\mathcal{N}=1$ Yang-Mills would require setting at least three ranks to zero, which is the number of degrees of freedom we have for the ranks. It turns out that this is possible. In particular set $N_{2}=N_{5}=N$ and $N_{1}=N_{3}=N_{4}=0$. Thus we expect a one parameter moduli of deformation and this is 
indeed the case. The geometry of the deformed space admits one compact 3-cycle and is given as follows.

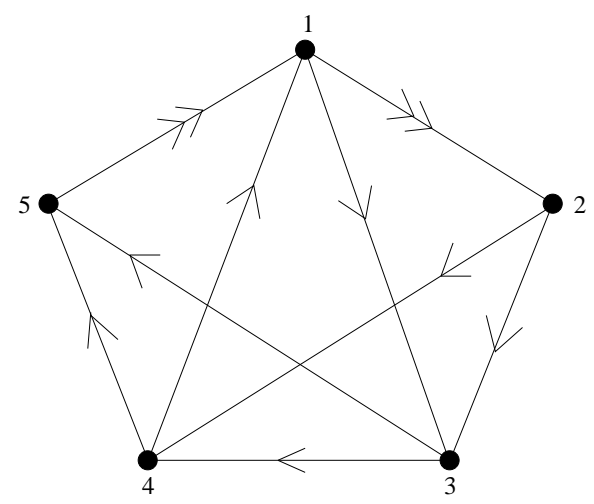

Figure 17:Quiver diagram for the field theory in the $B_{2}$ case.

Here and later we will use a general model for the blown-down geometry of $B_{k}$ : for $k \leq 6$ there is an (anticanonical) embedding of $B_{k}$ into $\mathbf{P}^{9-k}$. Thinking of $\mathbf{P}^{9-k}$ as the projectivization of $\mathbf{C}^{10-k}$, the embedded $B_{k}$ is the projectivization of its associated cone, which is a three dimensional variety in $\mathbf{C}^{10-k}$, singular at the vertex of the cone. This is a local model for the geometry, the del Pezzo being contracted to obtain the singular vertex.

The idea used in constructing the deformation is to find a bigger cone $C$ such that the above local model can be constructed from $C$ by imposing the vanishing of certain linear equations vanishing at the vertex. The deformation is obtained by simply deforming these linear equations away from the vertex. We now illustrate with $k=2$, where the local model is a cone in $\mathbf{C}^{8}$.

We start by noticing that $B_{2}$ can be described as the blowup of $\mathbf{P}^{1} \times \mathbf{P}^{1}$ at one point, and that $\mathbf{P}^{1} \times \mathbf{P}^{1}$ is a degree 2 hypersurface in $\mathbf{P}^{3}$. It follows that $B_{2}$ is a hypersurface in the blowup of $\mathbf{P}^{3}$ at a point, which we may as well take to be $(1,0,0,0)$. The hypersurface has degree 2 in the variables of $\mathbf{P}^{3}$ and contains $(1,0,0,0)$. Such a quadratic polynomial is a linear combination $\sum a_{i} m_{i}$ of the 9 monomials

$$
x_{1} x_{2}, x_{1} x_{3}, x_{1} x_{4}, x_{2}^{2}, x_{2} x_{3}, x_{2} x_{4}, x_{3}^{2}, x_{3} x_{4}, x_{4}^{2}
$$

Said differently, these 9 monomials define the coordinates of a mapping $\phi$ from the blowup of $\mathbf{P}^{3}$ to $\mathbf{P}^{8}$, and $B_{2}$ is a hyperplane section of the image of $\phi$. In the coordinates $\left(y_{1}, \ldots, y_{9}\right)$ of $\mathbf{P}^{8}$, the image of $\phi$ is defined by quadratic equations such as $y_{1} y_{5}=y_{2} y_{4}, y_{1} y_{6}=$ $y_{3} y_{4}, \ldots$ If these equations are viewed as equations in $\mathbf{C}^{9}$, the result is a 4 dimensional 
variety in $\mathbf{C}^{9}$ which is the cone over the image of $\phi$. Taking the hyperplane section $\sum a_{i} y_{i}=0$ containing the vertex, we get the local model inside the hyperplane which is identified with $\mathbf{C}^{8}=\mathbf{C}^{10-k}$ and we have recovered the general construction concretely. The vertex of the cone is identified with the singular point after contracting $B_{2}$. The transition is completed by smoothing this singularity. The deformation is given by

$$
\sum_{i} a_{i} y_{i}=t
$$

where $t$ is a deformation parameter. For $t=0$, this is the blown-down geometry; for general $t$, this is a smooth threefold. The size of $t$ is determined, using the minimization of the superpotential, as discussed before. It will change depending on the single integer $N$ controlling the rank of the disconnected gauge groups.

\section{$B_{3}$ and transition}

This is the case which was studied in [20,77]. In particular four inequivalent models related to each other by Seiberg duality were studied there. In the notation of [20] consider these theories, shown in Fig. 18 below.
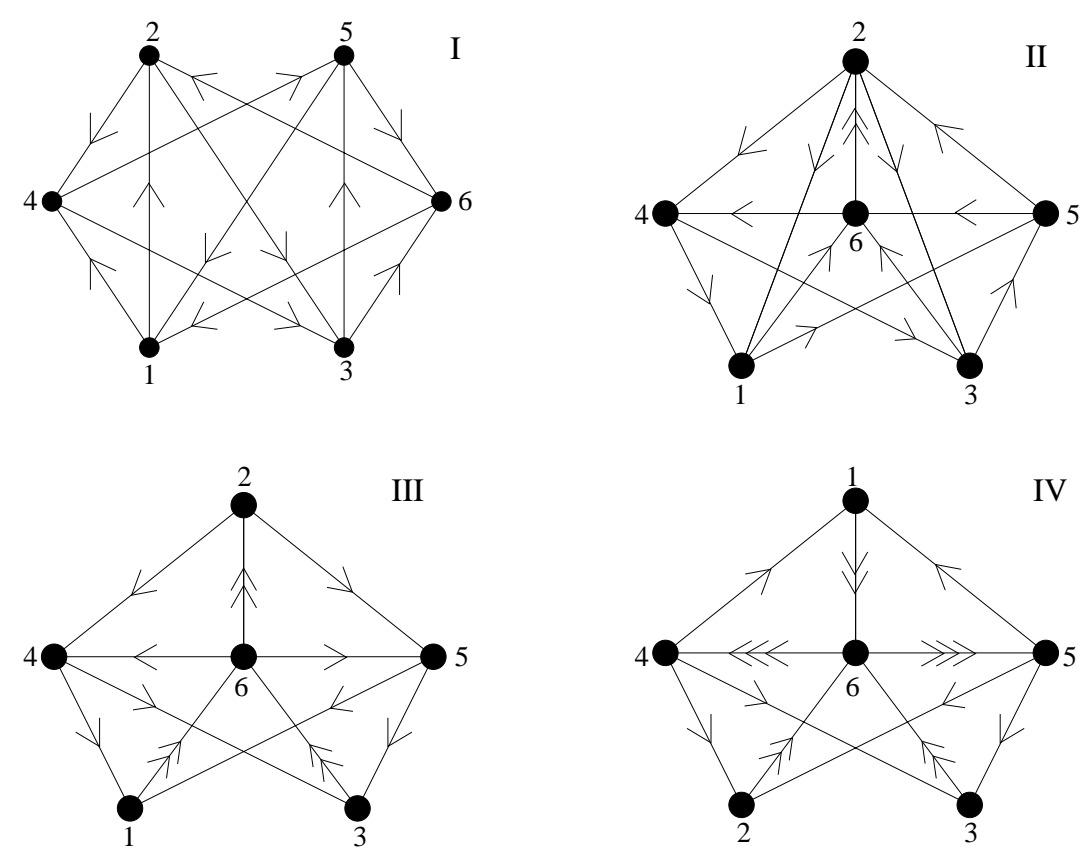

Figure 18: Quiver diagrams of the four different field theory realizations in the $B_{3}$ case. These are related by Seiberg duality. 
The conditions for anomaly cancellation can be written for these models. As expected there are four independent integers which satisfy anomaly free condition as expected. We can write the two conditions, for example, as

$$
\begin{gathered}
\text { Model } I: N_{5}=N_{1}+N_{2}-N_{3}, \quad N_{6}=N_{3}+N_{4}-N_{1} . \\
\text { Model II }: N_{5}=2 N_{2}+N_{4}-N_{1}-N_{3}, \quad N_{6}=N_{1}+N_{3}-N_{2} \\
\text { Model III }: N_{5}=2\left(N_{1}+N_{3}-N_{2}\right)-N_{4}, \quad N_{6}=N_{1}+N_{3}-N_{2} \\
\text { Model IV }: N_{5}=\frac{2}{3}\left(N_{1}+N_{2}+N_{3}\right)-N_{4}, \quad N_{6}=\frac{1}{3}\left(N_{1}+N_{2}+N_{3}\right) .
\end{gathered}
$$

These theories can be represented by sheaves as discussed before. For example model III corresponds to

$$
\begin{array}{ll}
1: & \mathcal{O}\left(e_{3}\right) \\
2: & \mathcal{O}\left(e_{1}\right) \\
3: & \mathcal{O}\left(e_{2}\right) \\
4: & \mathcal{O}\left(H-e_{1}\right) \\
5: & \mathcal{O}\left(H-e_{2}\right) \\
6: & \mathcal{O}(H)
\end{array}
$$

where $e_{i}$ denotes the class of the three blowups and $H$ is the hyperplane class. Also we have to reverse the orientation of 2,4 and 5 .

For the case studied in [20,57] where the $N_{i}$ are all equal, it was shown that one can go through dualizing node 1 of model I to get model II, then dualizing node 5 to get model III and then dualizing node 2 to get model IV. Of course these dualities all immediately generalize to the case where the $N_{i}$ are not equal with suitable superpotentials, as discussed in full generality before.

As an example, consider model III. Dualizing node 1 takes one back to model III, with a change conjugation and relabeling of nodes $2 \leftrightarrow 3$. The resulting theory has $N_{1}^{\prime}=N_{4}+N_{5}-N_{1}, N_{2}^{\prime}=N_{3}, N_{3}^{\prime}=N_{2}$ and $N_{4} \ldots N_{6}$ unchanged. In all of the models, one could also dualize the nodes having more than two arrows in and two out, and this would generate duals with quivers not appearing in fig. 18.

For general $N_{i}$ 's, we'll have an analog of the cascading flow to the IR. For some of the possible endpoints of these flows we could get e.g. model I with only the disconnected nodes $N_{4}=N_{6}$ non-zero (or only $N_{1}=N_{3}$ non-zero, or only $N_{2}=N_{5}$ non-zero). For 
this end point we have a product of pure Yang-Mills, leading to gaugino condensation, controlled by one integer. Another example of an endpoint is model III with only the disconnected nodes $N_{1}, N_{2}, N_{3}$ non-zero with $N_{2}=N_{1}+N_{3}$, which has a two parameter choice. This corresponds to the class given by

$$
N_{1}\left(e_{3}\right)-N_{2}\left(e_{1}\right)+N_{3}\left(e_{2}\right)=N_{1}\left(e_{3}-e_{1}\right)+N_{3}\left(e_{2}-e_{1}\right)
$$

which is orthogonal to $c_{1}=3 H-e_{1}-e_{2}-e_{3}$. Thus in this case we would expect to have a transition involving two independent blown up 3-cycles, controlled by two complex parameters. Thus from the quiver analysis we are led to expect transitions involving either one or 2 dimensional moduli space. This is indeed the case!

We can embed $B_{3}$ in $\mathbf{C}^{10-k}=\mathbf{C}^{7}$ as before. But this can be realized as a hyperplane section of a 4 dimensional variety in $\mathbf{C}^{8}$ as well as the intersection of two hyperplanes with a 5 dimensional variety in $\mathbf{C}^{9}$. The deformations are obtained by simply deforming the hyperplanes to avoid the vertex. There are as many deformation parameters as there are hyperplanes.

One-dimensional component: Embed $\mathbf{P}^{1} \times \mathbf{P}^{1} \times \mathbf{P}^{1}$ in $\mathbf{P}^{7}$ using the 8 multilinear polynomials on $\mathbf{P}^{1} \times \mathbf{P}^{1} \times \mathbf{P}^{1}$. A general hyperplane section is identified with $B_{3}$ in $\mathbf{P}^{6}$. Then take the cone over the 3 dimensional image to get a 4 dimensional singular variety in $\mathbf{C}^{8}$. A hyperplane section through the vertex is identified with the blown-down geometry in $\mathbf{C}^{7}=\mathbf{C}^{10-k}$. The deformation is $f\left(x_{1}, \ldots, x_{8}\right)=t$, where $f$ is a fixed homogeneous linear polynomial.

Two-dimensional component: Embed $\mathbf{P}^{2} \times \mathbf{P}^{2}$ in $\mathbf{P}^{8}$ using the 9 bilinear polynomials. Intersecting with 2 hyperplanes yields $B_{3}$. Take the cone to get a singular 5 dimensional variety in $\mathbf{C}^{9}$. Now the deformation is given by taking 2 linear polynomials:

$$
g\left(x_{1}, \ldots, x_{9}\right)=t_{1} \quad h\left(x_{1}, \ldots, x_{9}\right)=t_{2},
$$

a deformation in $\mathbf{C}^{7}$.

Of course in all these cases we can put the fluxes on the dual geometry to find the exact sizes for the blown up $S^{3}$ 's, as is by now rather familiar. This yields exact results for such $\mathcal{N}=1$ quiver theories, for which no other method is known. Also we can cascade backwards as in the $\mathbf{P}^{1} \times \mathbf{P}^{1}$ example already discussed, where as we go up in the cascade the number of 3-branes continues to increase. This class of examples illustrates that not only can these chiral theories undergo gaugino condensate, but that they can also be viewed as arising from an infinite tower of cascades. 


\subsection{The general del Pezzo case and affine $E_{k}$ symmetry}

Now let us analyze the more general case of $P^{2}$ blown up at $k$ points with $k=$ $4, \ldots, 8$. Even though these are not toric, one can construct their quiver diagram and the superpotential terms from the study of the associated exceptional collection, as we have discussed (the quiver diagrams for these classes has been proposed in [57]) . Our comments also apply to the cases with $k<4$ (with suitable definitions for $E_{k}$ ). The transitions in this case have also been considered [75] and the result is that there are $C_{2}-1$ deformations where $C_{2}$ is the dual coxeter number of $E_{k}$ groups with $E_{4}=A_{4}, E_{5}=D_{5}$. In particular the $E_{k}$ Weyl group is quite relevant for such geometries as we will explain below.

Let $x$ denote an $H_{2}$ class of $B_{k}$ and let $c_{1}$ denote a class in $H_{2}$ dual to the chern class of $B_{k}$. We know that the total allowed charge we have is given by an element $x$ such that

$$
x \cdot c_{1}=0
$$

This yields a $k$ dimensional integral space, which gives all the allowed 5-brane charges. This space, which is $k$-dimensional, turns out to be given by the root lattice of $E_{k}$.

Let us denote the blow up classes by $e_{i}$ and the hyperplane class by $H$. These have a Lorentzian self-intersection

$$
H \cdot H=1 \quad H \cdot e_{i}=0 \quad e_{i} \cdot e_{j}=-\delta_{i j}
$$

and $c_{1}=3 H-\sum_{i} e_{i}$. A basis for the simple roots of $E_{k}$ can be chosen to be $H-e_{1}-e_{2}-e_{3}$ and $e_{i}-e_{i+1}$ as $i=1, \ldots, k-1$. One can see that these span the root lattice of $E_{k}$ with the canonical inner product (up to an overall sign). More is in fact true. The automorphism of del Pezzo cohomology is given by the Weyl group of $E_{k}$. In particular for any such root $x$ the action on another class is given by

$$
y \rightarrow y+(y \cdot x) x
$$

That is very much in the spirit of what we studied in the context of A-D-E spaces.

There is also a natural set of spherical bundles, which are related to the weight lattice of the $E_{k}$. Namely, consider the classes $x$ such that

$$
x \cdot c_{1}=p
$$

for some fixed $p$. Note that the difference of any such $x$ 's is on the root lattice. Moreover $x \cdot($ root lattice $) \in \mathbf{Z}$ which implies that $x$ is in the weight lattice of $E_{k}$. For $p=1$ the 
corresponding $x$ are given by the weights of the fundamental representation of the $E_{k}$ (except for $E_{8}$ which gives the 240 roots). Note that all the line bundles corresponding to $\mathcal{O}(x)$ correspond to spherical bundles, and so the corresponding brane would yield an $\mathcal{N}=1$ Yang-Mills theory with no adjoint fields. If we consider branes corresponding to such sheaves, the Weyl reflection by the root vectors acts on the underlying quiver theory, as that is related to the automorphism of the del Pezzo, very much like how it arose in the A-D-E quiver theory. Also adding the 3-branes to the story should promote the $E_{k}$ to the affine version.

Suppose we now want to study generally what are the allowed phases for these theories yielding pure $\mathcal{N}=1$ gauge theory leading to gaugino condensates. We would have to find the allowed branches of these theories and look for spherical bundles with no matter between them. Note that if $R$ is a root, then the bundle $\mathcal{O}(R)$ has no cohomology. Since $-R$ is also a root, then the dual bundle $\mathcal{O}(-R)=\mathcal{O}(R)^{*}$ has no cohomology either. So given a collection of bundles $V_{\alpha}$ such that each $V_{\alpha}^{*} \otimes V_{\beta}$ is a root for all $\alpha \neq \beta$, then the corresponding nodes in the quiver are totally disconnected.

We now ask: what is the cardinality of the maximal set of bundles such that each pair differs by a root in the above sense? Or more generally, differs by something with only 1 cohomology? It is not difficult to see that there are at most $k+1$ such choices. For example, in the $B_{4}$ case we have $C_{2}=5$ bundles which we write symmetrically as

$$
H-e_{1}, H-e_{2}, H-e_{3}, H-e_{4}, 2 H-e_{1}-e_{2}-e_{3}-e_{4}
$$

This somewhat cumbersome form reflects our method for obtaining these bundles: by finding geometric curve classes $x$ satisfying (13.1) with $p=2$. The difference are all of the form $e_{i}-e_{j}$ or $\pm\left(H-e_{i}-e_{j}-e_{k}\right)$, all of which are roots. So in this case, we have $5=C_{2}$ disjoint bundles. The number drops to 4 after requiring orthogonality to $c_{1}$, which is the expected number of deformations of $B_{4}$ namely $C_{2}-1=4$. This also works in the $B_{3}, B_{2}$ and $\mathbf{P}^{1} \times \mathbf{P}^{1}$ cases that we already discussed. For example in the $B_{3}$ case we have $E_{3}=A_{1}+A_{2}$ and the two branches that we found correspond to weights which differ either by the root of $A_{1}$ (the one dimensional branch) or two roots of $A_{2}$ (the two dimensional branch). The $\mathbf{P}^{1} \times \mathbf{P}^{1}$ case corresponds to $E_{1}=A_{1}$ and that is the case where the relevant class corresponds to the difference of the two $\mathbf{P}^{1}$ 's as we have discussed. Note that in this case the affine $E_{1}=A_{1}$ action is indeed realized as was discussed before. In particular the $A_{1}$ Weyl reflection is generated by the class given by the difference of the two $\mathbf{P}^{1}$ 's. 
However we encounter the puzzle for higher $k$ 's that there are less pure $\mathcal{N}=1$ branches that we have identified, than predicted from the deformations. In particular $C_{2}-1>k$

for $E_{k}$ with $k>4$. Note that these would be the cases where on the A-D-E quiver theories we would also have the non-abelian branches. It would be interesting to understand how these extra branches would appear in the cases of these del Pezzos. One would naturally expect them to correspond to higher rank bundles.

\section{Acknowledgements}

We would like to thank M. Aganagic, O. Aharony, M. Berkooz, D.-E. Diaconescu, M.R. Douglas, A. Elezi, K. Hori, A. Iqbal, R. Leigh, E. Sharpe and E. Zaslow for valuable discussions. BF would also like to thank Harvard University for hospitality during part of this project.

The research of FC and CV is supported in part by NSF grants PHY-9802709 and DMS-0074329. The research of BF is supported by a Marie Curie fellowship, and also by the IRF Centers of Excellence program, by the European RTN network HPRN-CT-200000122, and by Minerva. The research of KI is supported by DOE-FG03-97ER40546. The research of SK is supported in part by NSF grant DMS-073657 and NSA grant MDA90400-1-052. 


\section{References}

[1] A. Strominger, "Massless Black Holes and Conifolds in String Theory," Nucl.Phys. B451 (1995) 96-108 [hep-th/9504090].

[2] B. Greene, D. Morrison and A. Strominger, "Black Hole Condensation and the Unification of String Vacua," Nucl.Phys. B451 (1995) 109-120 [ hep-th/9504145].

[3] M. Bershadsky, A Johansen, T. Pantev, V. Sadov and C. Vafa, "F-theory, Geometric Engineering and N=1 Dualities," Nucl.Phys.B505 (1997) 153-164 [hep-th/9612052]; C. Vafa and B. Zwiebach, "N=1 Dualities of SO and USp Gauge Theories and TDuality of String Theory," Nucl.Phys. B506 (1997) 143-156 [hep-th/9701015].

[4] H. Ooguri and C. Vafa, "Geometry of $\mathrm{N}=1$ Dualities in Four Dimensions," Nucl.Phys. B500 (1997) 62-74 hep-th/9702180.

[5] O. Aharony, S.S. Gubser, J. Maldacena, H. Ooguri and Y. Oz, "Large N Field Theories, String Theory and Gravity," Phys.Rept. 323 (2000) 183-386 hep-th/9905111].

[6] R. Gopakumar and C. Vafa, "On the Gauge Theory/Geometry Correspondence," Adv.Theor.Math.Phys. 3 (1999) 1415-1443 hep-th/9811131.

[7] C. Vafa, "Superstrings and Topological Strings at Large N," hep-th/0008142.

[8] B.S. Acharya, "On Realising N=1 Super Yang-Mills in M theory," hep-th/0011089.

[9] M. Atiyah, J. Maldacena and C. Vafa," An M-theory Flop as a Large N Duality," hep-th/0011256.

[10] M. Aganagic and C. Vafa, "Mirror Symmetry and a $G_{2}$ Flop," hep-th/0105225.

[11] M. Atiyah and E. Witten, " M-Theory Dynamics On A Manifold Of $G_{2}$ Holonomy," hep-th/0107177.

[12] I. Klebanov and M. Strassler, "Supergravity and a Confining Gauge Theory: Duality Cascades and $\chi$ SB-Resolution of Naked Singularities," JHEP 0008 (2000) 052 hepth/0007191].

[13] F. Cachazo, K. Intriligator and C. Vafa, "A Large N Duality via a Geometric Transition," Nucl.Phys. B603 (2001) 3-41 hep-th/0103067.

[14] J. Edelstein, K. Oh, and R. Tatar, "Orientifold, Geometric Transition and Large N Duality for SO/Sp Gauge Theories," JHEP 0105 (2001) 009 hep-th/0104037;

K. Dasgupta, K. Oh, and R. Tatar, "Geometric Transition, Large N Dualities and MQCD Dynamics," hep-th/0105066 ;

K. Dasgupta, K. Oh, and R. Tatar, "Open/Closed String Dualities and Seiberg Duality from Geometric Transitions in M-theory," hep-th/0106040].

[15] S. Elitzur, A. Giveon, D. Kutasov, E. Rabinovici and A. Schwimmer, "Brane Dynamics and N=1 Supersymmetric Gauge Theory," Nucl.Phys. B505 (1997) 202-250 hep-th/9704104.

[16] P. Argyres, M. Ronen Plesser and N. Seiberg, "The Moduli Space of N=2 SUSY QCD and Duality in N=1 SUSY QCD," Nucl. Phys. B 471, 159 (1996) hep-th/9603042]. 
[17] F. Cachazo, S. Katz, and C. Vafa, "Geometric Transitions and N=1 Quiver Theories," hep-th/0108120

[18] S. Katz, P. Mayr and C. Vafa, "Mirror symmetry and Exact Solution of 4D N=2 Gauge Theories I," Adv.Theor.Math.Phys. 1 (1998) 53-114 hep-th/9706110.

[19] K. Hori, A. Iqbal and C. Vafa, "D-Branes And Mirror Symmetry," [hep-th/0005247].

[20] C. Beasley and M. Plesser, "Toric Duality Is Seiberg Duality," hep-th/0109053.

[21] B. Feng, A. Hanany, Y. He, A. Uranga, "Toric Duality as Seiberg Duality and Brane Diamonds," hep-th/0109063.

[22] S. Gubser, N. Nekrasov and S. Shatashvili, "Generalized conifolds and four dimensional N = 1 superconformal theories," JHEP 9905, 003 (1999) hep-th/9811230.

[23] I. Klebanov and E. Witten, "Superconformal Field Theory on Threebranes at a CalabiYau Singularity," Nucl.Phys. B536 (1998) 199-218 hep-th/9807080].

[24] N. Seiberg, "Electric - magnetic duality in supersymmetric nonAbelian gauge theories," Nucl. Phys. B 435, 129 (1995) hep-th/9411149.

[25] M. Aganagic, A. Karch, D. Lust, A. Miemiec, "Mirror Symmetries for Brane Configurations and Branes at Singularities," Nucl.Phys. B569 (2000) 277-302 hepth/9903093].

[26] K. Dasgupta, K. Oh, R. Tatar, "Open/Closed String Dualities and Seiberg Duality from Geometric Transitions in M-theory," hep-th/0106040.

[27] I. Antoniadis and B. Pioline, "Higgs branch, HyperKahler quotient and duality in SUSY N=2 Yang-Mills theories," Int.J.Mod.Phys. A12 (1997) 4907-4932 hepth/9607058].

[28] M. Douglas and G. Moore, "D-branes, Quivers, and ALE Instantons," hep-th/9603167.

[29] J.D. Blum and K. Intriligator, "New phases of string theory and 6d RG fixed points via branes at orbifold singularities," Nucl. Phys. B 506, 199 (1997) [hep-th/9705044].

[30] K. Intriligator, "New string theories in six dimensions via branes at orbifold singularities," Adv. Theor. Math. Phys. 1, 271 (1998) hep-th/9708117.

[31] E. Witten, "Solutions Of Four-Dimensional Field Theories Via M Theory," Nucl.Phys. B500 (1997) 3-42 [hep-th/9703166.

[32] A. Lawrence, N. Nekrasov and C. Vafa, "On Conformal Theories in Four Dimensions," Nucl.Phys. B533 (1998) 199-209 hep-th/9803015.

[33] D. Kutasov, A. Schwimmer and N. Seiberg, "Chiral Rings, Singularity Theory and Electric-Magnetic Duality," Nucl. Phys. B 459, 455 (1996) [hep-th/9510222].

[34] M. Shifman and A. Vainshtein, Nucl. Phys. B277 (1986) 456; Nucl. Phys. B539 (1991) 571; V. Novikov, M. Shifman, A. Vainshtein, and V. Zakharov, Nucl. Phys. B229 (1983) 381.

[35] S. Katz and D. Morrison, "Gorenstein Threefold Singularities with Small Resolutions via Invariant Theory for Weyl Groups", J. Algebraic Geometry 1 (1992) 449-530. 
[36] J. Maldacena and C. Nunez, "Towards the large $\mathrm{N}$ limit of pure $\mathrm{N}=1$ super Yang Mills," Phys.Rev.Lett. 86 (2001) 588-591 [hep-th/0008001].

[37] S. Gukov, C. Vafa and E. Witten, "CFT's From Calabi-Yau Four-folds," Nucl.Phys. B584 (2000) 69-108 hep-th/9906070; A. Shapere and C. Vafa, "BPS Structure of Argyres-Douglas Superconformal Theories," hep-th/9910182.

[38] T. Taylor and C. Vafa, "RR Flux on Calabi-Yau and Partial Supersymmetry Breaking," Phys.Lett. B474 (2000) 130-137 hep-th/9912152.

[39] P. Mayr, "On Supersymmetry Breaking in String Theory and its Realization in Brane Worlds," Nucl.Phys. B593 (2001) 99-126 [hep-th/0003198].

[40] V. Arnold, S. Gusein-Zade and A. Varchenko, "Singularities of Differentiable Maps," Vol. II, Monographs in Mathematics, vol. 82, Birkhäuse, Boston, Basel, Stuttgart, 1985.

[41] K. Intriligator and N. Seiberg, "Duality, monopoles, dyons, confinement and oblique confinement in supersymmetric $\mathrm{SO}(\mathrm{N}(\mathrm{c}))$ gauge theories," Nucl. Phys. B 444, 125 (1995) hep-th/9503179.

[42] G. Carlino, K. Konishi and H. Murayama, "Dynamical symmetry breaking in supersymmetric $\operatorname{SU}(\mathrm{n}(\mathrm{c}))$ and $\mathrm{USp}(2 \mathrm{n}(\mathrm{c}))$ gauge theories," Nucl. Phys. B 590, 37 (2000) hep-th/0005076.

[43] K. Hori, H. Ooguri and Y. Oz, "Strong Coupling Dynamics of Four-Dimensional N=1 Gauge Theories from M Theory Fivebrane", Adv.Theor.Math.Phys. 1 (1998) 1-52 hep-th/9706082.

[44] O. Aharony, J. Sonnenschein and S. Yankielowicz, "Flows and duality symmetries in N=1 supersymmetric gauge theories," Nucl. Phys. B 449, 509 (1995) hepth/9504113].

[45] S. Elitzur, A. Giveon, D. Kutasov, E. Rabinovici and A. Schwimmer, "Brane dynamics and N = 1 supersymmetric gauge theory," Nucl. Phys. B 505, 202 (1997) hep-th/9704104.

[46] D. Kutasov, "A Comment on duality in $\mathrm{N}=1$ supersymmetric nonAbelian gauge theories," Phys. Lett. B 351, 230 (1995) hep-th/9503086.

[47] D. Kutasov and A. Schwimmer, "On duality in supersymmetric Yang-Mills theory," Phys. Lett. B 354, 315 (1995) hep-th/9505004.

[48] S. Giddings, S. Kachru and J. Polchinski, "Hierarchies from Fluxes in String Compactifications," hep-th/0105097.

[49] R. Leigh and M. Strassler, "Exactly marginal operators and duality in fourdimensional N=1 supersymmetric gauge theory," Nucl. Phys. B 447, 95 (1995) thepth/9503121.

[50] D. Anselmi, J. Erlich, D. Freedman and A. Johansen, "Positivity constraints on anomalies in supersymmetric gauge theories," Phys. Rev. D 57, 7570 (1998) hepth/9711035]. 
[51] J. Polchinski, "Scale And Conformal Invariance In Quantum Field Theory," Nucl. Phys. B 303, 226 (1988).

[52] S. S. Gubser, Phys. Rev. D 59, 025006 (1999) [hep-th/9807164].

[53] A. Bergman and C. P. Herzog, hep-th/0108020.

[54] E. Witten, "Phases of $N=2$ Theories In Two Dimensions," Nucl.Phys. B403 (1993) 159-222 hep-th/9301042.

[55] K. Hori and C. Vafa, "Mirror Symmetry," hep-th/0002222.

[56] A. Klemm, W. Lerche, P. Mayr, C.Vafa and N. Warner, "Self-Dual Strings and N=2 Supersymmetric Field Theory," Nucl.Phys. B477 (1996) 746-766 hep-th/9604034.

[57] A. Hanany and A. Iqbal, "Quiver Theories from D6-branes via Mirror Symmetry," hep-th/0108137.

[58] A. Strominger, S.T. Yau and E. Zaslow, "Mirror Symmetry is T-Duality," Nucl.Phys. B479 (1996) 243-259 [hep-th/9606040].

[59] S. Cecotti, P. Fendley, K. Intriligator and C. Vafa, "A New Supersymmetric Index," Nucl.Phys. B386 (1992) 405-452 hep-th/9204102.

[60] M. Douglas, B. Fiol and C. Rmelsberger, "Stability and BPS branes," hepth/0002037.

[61] P. Seidel and R. Thomas, "Braid group actions on derived categories of coherent sheaves", Duke Math. Jour. 108 (2001) 37-108 math.AG/0001043.

[62] G. Gibbons and P. Townsend, "A Bogomol'nyi equation for intersecting domain walls," Phys.Rev.Lett. 83 (1999) 1727-1730 hep-th/9905196;

S. Carroll, S. Hellerman, M. Trodden, "Domain Wall Junctions are 1/4-BPS States," Phys.Rev. D61 (2000) 065001 hep-th/9905217];

P. Townsend, "PhreMology-calibrating M-branes," Class.Quant.Grav. 17 (2000) 1267-1276 hep-th/9911154;

S. Nam and K. Olsen, "Domain Wall Junctions in Supersymmetric Field Theories in $\mathrm{D}=4$," JHEP 0008 (2000) 001 [hep-th/0002176].

[63] M. Douglas, B. Greene and D. Morrison, "Orbifold Resolution by D-Branes," Nucl.Phys. B506 (1997) 84-106 hep-th/9704151.

[64] S. Cecotti and C. Vafa, "On Classification of N=2 Supersymmetric Theories," Commun.Math.Phys. 158 (1993) 569-644 hep-th/9211097.

[65] E. Zaslow, "Solitons and Helices: The Search for a Math-Physics Bridge," Commun.Math.Phys. 175 (1996) 337-376 hep-th/9408133.

[66] M. Douglas and G. Moore, "D-branes, Quivers, and ALE Instantons," [hep-th/9603167.

[67] S. Kachru, E. Silverstein, "4d Conformal Field Theories and Strings on Orbifolds," Phys.Rev.Lett. 80 (1998) 4855-4858 hep-th/9802183.

[68] D.-E. Diaconescu and J. Gomis, "Fractional branes and boundary states in orbifold theories", hep-th/9906242. 
[69] M. R. Douglas, B. Fiol and C. Römelsberger, "The spectrum of BPS branes on a noncompact Calabi-Yau", hep-th/0003263.

[70] D. Morrison and M. Plesser, "Non-Spherical Horizons, I," Adv.Theor.Math.Phys. 3 (1999) 1-81 hep-th/9810201.

[71] B. Feng, A. Hanany, Y. He, "D-Brane Gauge Theories from Toric Singularities and Toric Duality," Nucl.Phys. B595 (2001) 165-200

[72] R. Gopakumar and C. Vafa, "Branes and Fundamental Groups," Adv. Theor. Math. Phys. 2 (1998) 399-411 hep-th/9712077.

[73] B. Acharya and C. Vafa, "On Domain Walls of $N=1$ Supersymmetric Yang-Mills in Four Dimensions," hep-th/0103011.

[74] K. Altmann, "The versal deformation of an isolated toric Gorenstein singularity", Inv. Math. 128 (1997) 443-479 alg-geom/9403004.

[75] M. Gross, "Deforming Calabi-Yau Threefolds," alg-geom/9506022.

[76] C. Beasley, B. Greene, C. Lazaroiu and M. Plesser, "D3-branes on partial resolutions of abelian quotient singularities of Calabi-Yau threefolds," Nucl.Phys. B566 (2000) 599-640 hep-th/9907186

[77] B. Feng, A. Hanany, Y. He, " Phase Structure of D-brane Gauge Theories and Toric Duality," JHEP 0108 (2001) 040 hep-th/0104259 